\title{
TORIC HOMOTOPY THEORY
}

\author{
Stephen Theriault \\ Mathematical Sciences \\ University of Southampton \\ Southampton SO17 1BJ, UK \\ S.D.Theriault@soton.ac.uk
}

\begin{abstract}
These notes describe some of the homotopy theory surrounding DavisJanuszkiewicz spaces, moment-angle complexes and their generalizations to polyhedral products. These spaces are defined by gluing together products formed from pairs of spaces $(X, A)$, where the gluing is determined by the faces of a simplicial complex $K$. The emphasis is on determining homotopy types - of the spaces themselves, their suspensions and their based loop spaces - and showing how these homotopy types depend on a beautiful interplay between the topology of the pairs $(X, A)$ and the combinatorics of the simplicial complex $K$.
\end{abstract}

\section{Contents}

1 Introduction

2 Davis-Januszkiewicz spaces, moment-angle complexes and polyhedral products

3 Fundamental properties of polyhedral products 6

3.1 Functorial properties

3.2 Retractions of $(\underline{X}, \underline{A})^{K}$ induced by full subcomplexes of $K$

3.3 Pushouts of simplicial complexes induce pushouts of polyhedral products

3.4 Fibrations and homotopy fibrations

3.5 An elementary loop space decomposition 14

4 The homotopy type of $\Sigma(\underline{X}, \underline{A})^{K}$

5 Building simplicial complexes and polyhedral products vertex by vertex

5.1 Building simplicial complexes

5.2 Building polyhedral products

6 Identifying the homotopy type of certain pushouts 
7 A strategy for identifying the homotopy type of $(\underline{C X}, \underline{X})^{K}$ for certain $K \quad 29$

8 Higher Whitehead products and minimal missing faces 35

8.1 Whitehead products $\quad 35$

8.2 Higher Whitehead products $\quad 37$

8.3 Relating higher Whitehead products to minimal missing faces $\quad 40$

9 A decomposition of the based loops on a polyhedral product 44

10 Exponents and Barratt's conjecture $\quad 49$

11 Elliptic and hyperbolic spaces $\quad 51$

12 Moore's conjecture $\quad 53$

13 More on elliptic and hyperbolic spaces, and Moore's conjecture $\quad 55$

14 Further problems $\quad 62$

$\begin{array}{ll}\text { References } & 64\end{array}$

\section{Introduction}

Toric topology is at the nexus of a wide range of mathematical subjects: algebraic topology, symplectic and algebraic geometry, combinatorics and commutative algebra. Homotopy theory has proved to be very useful in analyzing the fundamental spaces of toric topology: Davis-Januszkiewicz spaces $D J_{K}$, moment-angle complexes $\mathcal{Z}_{K}$, and their generalizations to polyhedral products $(\underline{X}, \underline{A})^{K}$. All three are defined in terms of products of topological spaces glued together at intersections as determined by the faces of a simplicial complex $K$. The topology of these spaces is fascinating, involving a beautiful interplay between homotopy theory and properties of simplicial complexes.

The purpose of these notes is to give an introduction to the homotopy theory surrounding Davis-Januszkiewicz spaces, moment-angle complexes and polyhedral products. In one direction, methods and tools are developed that give the reader a grounding in the techniques that have led to the main advances in the homotopy theory of toric topology over the past decade. In another direction, these methods are applied to address important questions and conjectures in homotopy theory.

The main goals are to identify the homotopy types of the spaces $D J_{K}$, $\mathcal{Z}_{K}$ and $(\underline{X}, \underline{A})^{K}$, the homotopy types of their suspensions $\Sigma D J_{K}, \Sigma \mathcal{Z}_{K}$ and $\Sigma(\underline{X}, \underline{A})^{K}$, and the homotopy types of their based loop spaces $\Omega D J_{K}$, $\Omega \mathcal{Z}_{K}$ and $\Omega(\underline{X}, \underline{A})^{K}$. General formulas for the homotopy type of $\Sigma(\underline{X}, \underline{A})^{K}$ and $\Omega(\underline{X}, \underline{A})^{K}$ will be discussed in Theorems 4.5 and 9.4 respectively. The homotopy type of $(\underline{X}, \underline{A})^{K}$ itself is more subtle, and only special cases will be considered. The loop space decomposition of $(\underline{X}, \underline{A})^{K}$ is used in the latter part of the notes to address problems related to major questions in homotopy theory: Barratt's conjecture, the elliptic-hyperbolic duality in 
rational homotopy theory, and Moore's conjecture. Along the way, various open problems will be pointed out in the hope of provoking interest.

\section{Davis-Januszkiewicz spaces, moment-angle complexes and polyhedral products}

The two fundamental objects in toric topology are Davis-Januszkiewicz spaces and moment-angle complexes. They were first constructed by Davis and Januszkiewicz [10] but were reformulated more conveniently by Buchstaber and Panov $[5,6]$. For a positive integer $m$, let $[m]=\{1, \ldots, m\}$. Let $K$ be a simplicial complex on the vertex set $[m]$. Let $T^{m}=\prod_{i=1}^{m} S^{1}$ be the $m$-fold torus and let $B T^{m}=\prod_{i=1}^{\infty} \mathbb{C} P^{\infty}$ be its classifying space. For a face $\sigma \in K$, let

$$
D J_{\sigma}=\prod_{i=1}^{m} Y_{i} \quad \text { where } \quad Y_{i}= \begin{cases}\mathbb{C} P^{\infty} & \text { if } i \in \sigma \\ * & \text { if } i \notin \sigma .\end{cases}
$$

The Davis-Januszkiewicz space $D J_{K}$ is defined by

$$
D J_{K}=\bigcup_{\sigma \in K} D J_{\sigma}
$$

Observe that $D J_{K}$ is a subspace of the product $\prod_{i=1}^{m} \mathbb{C} P^{\infty}$.

The moment-angle complex is constructed analogously. For a face $\sigma \in K$, let

$$
\mathcal{Z}_{\sigma}=\prod_{i=1}^{m} Y_{i} \quad \text { where } \quad Y_{i}=\left\{\begin{array}{l}
D^{2} \text { if } i \in \sigma \\
S^{1} \text { if } i \notin \sigma .
\end{array}\right.
$$

The moment-angle complex $\mathcal{Z}_{K}$ is defined by

$$
\mathcal{Z}_{K}=\bigcup_{\sigma \in K} \mathcal{Z}_{\sigma}
$$

Observe that $\mathcal{Z}_{K}$ is a subspace of the product $\prod_{i=1}^{m} D^{2}$. There is a canonical action of $T^{m}$ on $\prod_{i=1}^{m} D^{2}$ given by letting the circle in coordinate $i$ of $T^{m}$ act by rotation on the disc in coordinate $i$ of $\prod_{i=1}^{m} D^{2}$. This induces an action of $T^{m}$ on the subspace $\mathcal{Z}_{K}$. The orbit space of this action on $\mathcal{Z}_{K}$ is $D J_{K}$. Thus there is a homotopy fibration sequence

$$
T^{m} \longrightarrow \mathcal{Z}_{K} \longrightarrow D J_{K} \longrightarrow B T^{m} .
$$

The analogous definitions of $D J_{K}$ and $\mathcal{Z}_{K}$ suggest that there is something more functorial going on that ought to be investigated. The generalization was formulated independently in unpublished notes by Strickland 
and by Buchstaber and Panov [5], and first seriously investigated by Bahri, Bendersky, Cohen and Gitler [1]. The generalization is called a polyhedral product.

Let $K$ be a simplicial complex on $m$ vertices. For $1 \leq i \leq m$, let $\left(X_{i}, A_{i}\right)$ be a pair of pointed $C W$-complexes, where $A_{i}$ is a pointed subspace of $X_{i}$. Let $(\underline{X}, \underline{A})=\left\{\left(X_{i}, A_{i}\right)\right\}_{i=1}^{m}$ be the sequence of $C W$-pairs. Regard each simplex (face) $\sigma \in K$ as being identified with the sequence $\left(i_{1}, \ldots, i_{k}\right)$ where $i_{1}, \ldots, i_{k}$ are the vertices of $\sigma$ and $i_{1}<\cdots<i_{k}$. Let $(\underline{X}, \underline{A})^{\sigma}$ be the subspace of $\prod_{i=1}^{m} X_{i}$ defined by

$$
(\underline{X}, \underline{A})^{\sigma}=\prod_{i=1}^{m} Y_{i} \quad \text { where } \quad Y_{i}=\left\{\begin{array}{l}
X_{i} \text { if } i \in \sigma \\
A_{i} \text { if } i \notin \sigma .
\end{array}\right.
$$

The polyhedral product determined by $(\underline{X}, \underline{A})$ and $K$ is

$$
(\underline{X}, \underline{A})^{K}=\bigcup_{\sigma \in K}(\underline{X}, \underline{A})^{\sigma} \subseteq \prod_{i=1}^{m} X_{i} .
$$

In particular, if each pair of spaces $\left(X_{i}, A_{i}\right)$ equals $\left(\mathbb{C} P^{\infty}, *\right)$ then the polyhedral product $(\underline{X}, \underline{A})^{K}$ is the definition of the Davis-Januszkiewicz space $D J_{K}$. If each pair of spaces $\left(X_{i}, A_{i}\right)$ equals $\left(D^{2}, S^{1}\right)$ then the polyhedral product $(\underline{X}, \underline{A})^{K}$ is the definition of the moment-angle complex $\mathcal{Z}_{K}$. Thus determining homotopy theoretic information about polyhedral products in general lets one deduce properties of Davis-Januszkiewicz spaces and moment-angle complexes.

To get a feeling for polyhedral products, we consider several examples. Let $I$ be the unit interval and let $A$ and $B$ be pointed topological spaces. The reduced join of $A$ and $B$ is the quotient space

$$
A * B=(A \times I \times B) / \sim
$$

where $(a, 1, b) \sim\left(a^{\prime}, 1, b\right),(a, 0, b) \sim\left(a, 0, b^{\prime}\right)$ and $(*, t, *) \sim *$ for all $a, a^{\prime} \in A, b, b^{\prime} \in B$ and $t \in I$. It is well known that there is a natural homotopy equivalence $A * B \simeq \Sigma A \wedge B$.

Example 2.1: Suppose that $K$ is $m$ disjoint points. Then the faces of $K$ consist of the emptyset and the vertices. Since the emptyset has no vertices, from the definition of a polyhedral product we obtain

$$
(\underline{X}, \underline{A})^{\emptyset}=A_{1} \times \cdots \times A_{m} .
$$

Since the face $\{i\}$ consists only of one vertex, from the definition of a polyhedral product we obtain

$$
(\underline{X}, \underline{A})^{\{i\}}=A_{1} \times \cdots \times A_{i-1} \times X_{i} \times A_{i+1} \times \cdots \times A_{m} .
$$


As $A_{1} \times \cdots \times A_{m}$ is a subspace of $(\underline{X}, \underline{A})^{\{i\}}$ for all $1 \leq i \leq m$, we obtain

$$
(\underline{X}, \underline{A})^{K}=\bigcup_{i=1}^{m} A_{1} \times \cdots \times A_{i-1} \times X_{i} \times A_{i+1} \times \cdots \times A_{m} .
$$

In particular, if each $A_{i}$ equals the basepoint, then $A_{1} \times \cdots \times A_{m}=* \times \cdots \times *$, which equals the basepoint in the product. So

$$
(\underline{X}, \underline{A})^{K}=\bigvee_{i=1}^{m} X_{i}
$$

Example 2.2: Changing the pairs of spaces $\left(X_{i}, A_{i}\right)$ can have a dramatic effect on the homotopy type of the polyhedral product. For example, if $K$ is two disjoint points then as in Example 2.1 we have

$$
(\underline{X}, \underline{A})^{K}=X_{1} \times A_{2} \cup A_{1} \times X_{2}
$$

where the union is over $A_{1} \times A_{2}$. This may be better represented as a pushout

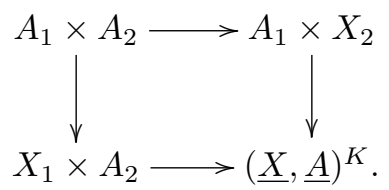

If $A_{1}$ and $A_{2}$ are the basepoints in $X_{1}$ and $X_{2}$ respectively, then $(\underline{X}, \underline{*})^{K} \simeq$ $X_{1} \vee X_{2}$. But if $X_{1}$ and $X_{2}$ are the reduced cones on $A_{1}$ and $A_{2}$ respectively, then as we shall see in Section 6, the pushout becomes that for the join

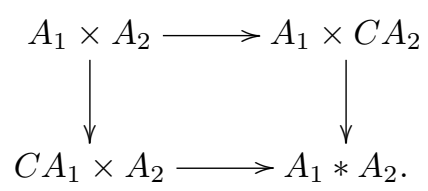

That is $(\underline{C A}, \underline{A})^{K} \simeq A_{1} * A_{2}$.

Example 2.3: At the other extreme, if $K=\Delta^{m-1}$ is the full $(m-1)$ simplex, then $\sigma=(1, \ldots, m)$ is a face of $K$, so from the definition of a polyhedral product we obtain

$$
(\underline{X}, \underline{A})^{\sigma}=X_{1} \times \cdots \times X_{m} .
$$

As $(\underline{X}, \underline{A})^{\sigma}$ is a subspace of $(\underline{X}, \underline{A})^{K}$, and $(\underline{X}, \underline{A})^{K}$ is a subspace of $\prod_{i=1}^{m} X_{i}$, we obtain

$$
(\underline{X}, \underline{A})^{\Delta^{m-1}}=X_{1} \times \cdots \times X_{m} .
$$

Note that this holds regardless of the subspaces $A_{i}$ in the pairs $\left(X_{i}, A_{i}\right)$. 
Example 2.4: Seemingly small changes in the simplicial complex can also result in dramatic changes in the homotopy type of the polyhedral product. Consider the simplicial complexes

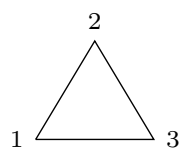

$K_{1}$

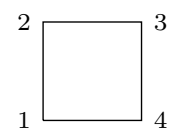

$K_{2}$

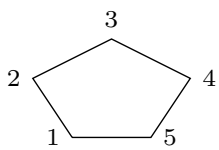

$K_{3}$

It is not obvious simply from the definition of a polyhedral product, but in these cases it turns out that $\mathcal{Z}_{K_{1}} \simeq S^{5}, \mathcal{Z}_{K_{2}} \simeq S^{3} \times S^{3}$ and $\mathcal{Z}_{K_{3}} \simeq$ $\left(S^{3} \times S^{4}\right)^{\# 5}$, where \# is the connected sum operation.

\section{Fundamental properties of polyhedral products}

In this section five fundamental properties of polyhedral products are discussed: functorial properties, retractions of $(\underline{X}, \underline{A})^{K}$ induced by full subcomplexes of $K$, how pushouts of simplicial complexes induce pushouts of polyhedral products, fibrations and homotopy fibrations, and an elementary loop space decomposition.

\subsection{Functorial properties}

There are two ingredients in defining a polyhedral product, the sequence of pairs $(\underline{X}, \underline{A})$ and the simplicial complex $K$. Both have functorial properties.

Proposition 3.1: The polyhedral product $(\underline{X}, \underline{A})^{K}$ satisfies the following:

(a) if there are maps of pairs $\left(X_{i}, A_{i}\right) \longrightarrow\left(X_{i}^{\prime}, A_{i}^{\prime}\right)$, for $1 \leq i \leq$ $m$ then for any simplicial complex $K$ on the vertex set $[m]$ there is an induced map of polyhedral products $(\underline{X}, \underline{A})^{K} \longrightarrow$ $\left(\underline{X^{\prime}}, \underline{A^{\prime}}\right)^{K}$

(b) if $K$ and $L$ are simplicial complexes on the vertex sets $[m]$ and $[n]$ respectively and there is an inclusion of simplicial complexes $K \longrightarrow L$, then for any sequence $(\underline{X}, \underline{A})$ of $n$ pairs of pointed, path-connected $C W$-complexes there is an induced map of polyhedral products $(\underline{X}, \underline{A})^{K} \longrightarrow(\underline{X}, \underline{A})^{L}$.

Proof: Let $\sigma$ be a face of $K$ and recall that $(\underline{X}, \underline{A})^{\sigma}=\prod_{i=1}^{m} Y_{i}$ where $Y_{i}=$ $X_{i}$ if $i \in \sigma$ and $Y_{i}=A_{i}$ if $i \notin \sigma$. The maps of pairs $\left(X_{i}, A_{i}\right) \longrightarrow\left(X_{i}^{\prime}, A_{i}^{\prime}\right)$ 
therefore induce a map of products

$$
(\underline{X}, \underline{A})^{\sigma}=\prod_{i=1}^{m} Y_{i} \longrightarrow \prod_{i=1}^{m} Y_{i}^{\prime}=\left(\underline{X^{\prime}}, \underline{A^{\prime}}\right)^{\sigma} .
$$

Taking the union over all $\sigma \in K$ we obtain a map

$$
(\underline{X}, \underline{A})^{K}=\bigcup_{\sigma \in K}(\underline{X}, \underline{A})^{\sigma} \longrightarrow \bigcup_{\sigma \in K}\left(\underline{X^{\prime}}, \underline{A^{\prime}}\right)^{\sigma}=\left(\underline{X^{\prime}}, \underline{A^{\prime}}\right)^{K} .
$$

This proves part (a).

For part (b), let $\sigma$ be a face of $K$. Since the map of simplicial complexes $f: K \longrightarrow L$ is an inclusion, $\sigma$ is also a face of $L$. Write $\sigma_{K}, \sigma_{L}$ for $\sigma$ viewed as a face of $K$ or $L$ respectively. The map $f$ sends the vertex $i \in[m]$ to $f(i) \in[n]$, so we obtain a map of products

$$
(\underline{X}, \underline{A})^{\sigma_{K}}=\prod_{i=1}^{m} Y_{i} \longrightarrow \prod_{i=1}^{m} Y_{f(i)} \hookrightarrow \prod_{j=1}^{n} Y_{j}=(\underline{X}, \underline{A})^{\sigma_{L}} .
$$

Taking the union over all the faces of $K$, we obtain

$$
(\underline{X}, \underline{A})^{K}=\bigcup_{\sigma_{K} \in K}(\underline{X}, \underline{A})^{\sigma_{K}} \longrightarrow \bigcup_{\sigma_{K} \in K}(\underline{X}, \underline{A})^{\sigma_{L}} \hookrightarrow \bigcup_{\sigma_{L} \in L}(\underline{X}, \underline{A})^{\sigma_{L}}=(\underline{X}, \underline{A})^{L} .
$$

Example 3.2: Regarding $\mathbb{C} P^{1}$ as $S^{2}$, there is an inclusion $S^{2} \longrightarrow \mathbb{C} P^{\infty}$. This induces a map of pairs $\left(S^{2}, *\right) \longrightarrow\left(\mathbb{C} P^{\infty}, *\right)$. Taking $m$ copies of the same pair, by Proposition 3.1 (a) and the definition of $D J_{K}$, there is a map of polyhedral products $\left(\underline{S^{2}}, \underline{*}\right)^{K} \longrightarrow\left(\underline{\mathbb{C} P^{\infty}}, \underline{*}\right)^{K}=D J_{K}$.

Example 3.3: Let $L$ be a simplicial complex on the vertex set $m$ and let $K$ be the set of vertices of $L$. Then there is an inclusion of simplicial complexes $K \longrightarrow L$. In the case of $(\underline{X}, \underline{*})$, by Example $2.1,(\underline{X}, \underline{*})^{K}=\bigvee_{i=1}^{m} X_{i}$. So by Proposition 3.1 (b) we obtain a map of polyhedral products $\bigvee_{i=1}^{m} X_{i} \longrightarrow$ $(\underline{X}, \underline{*})^{L}$.

\subsection{Retractions of $(\underline{X}, \underline{A})^{K}$ induced by full subcomplexes of $K$}

There are distinguished subcomplexes of a simplicial complex $K$ which play a special role in the homotopy theory of polyhedral products.

Definition 3.4: Let $K$ be a simplicial complex on the vertex set $[m]$. If $I \subseteq[m]$ then the full subcomplex $K_{I}$ of $K$ is defined as the simplicial complex

$$
K_{I}=\bigcup\{\sigma \in K \mid \text { the vertex set of } \sigma \text { is in } I\} .
$$


Example 3.5: Let $K$ be the simplical complex on 4 vertices pictured as follows:

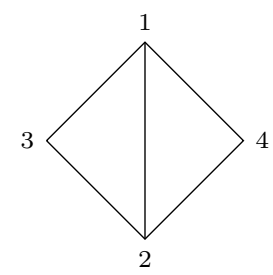

$K$

If $I_{1}=\{1,2,3\}, I_{2}=\{1,3,4\}$ and $I_{3}=\{3,4\}$ then the full subcomplexes $K_{I_{1}}, K_{I_{2}}$ and $K_{I_{3}}$ are:

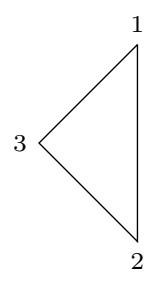

$K_{I_{1}}$

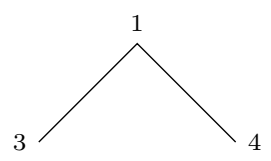

$K_{I_{2}}$

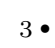

$K_{I_{3}}$

The definition of $K_{I}$ implies that the inclusion of $K_{I}$ into $K$ is a map of simplicial complexes. So by Proposition 3.1 (b) there is a map of polyhedral products $(\underline{X}, \underline{A})^{K_{I}} \longrightarrow(\underline{X}, \underline{A})^{K}$. However, projecting from $[m]$ to $I$ does not induce a map of simplicial complexes $K \longrightarrow K_{I}$. Yet on the level of polyhedral products, such a projection does exist. If $I=\left\{i_{1}, \ldots, i_{k}\right\}$ for $1 \leq i_{1}<\cdots<i_{k} \leq m$, let $X^{I}=\prod_{j=1}^{k} X_{i_{j}}$.

Proposition 3.6: Let $K$ be a simplicial complex on the vertex set $[\mathrm{m}]$ and let $(\underline{X}, \underline{A})$ be any sequence of pointed, path-connected $C W$-pairs. Let $I \subseteq[m]$. Then the following hold:

(a) the inclusion $X^{I} \longrightarrow \prod_{i=1}^{m} X_{i}$ induces a map $(\underline{X}, \underline{A})^{K_{I}} \longrightarrow(\underline{X}, \underline{A})^{K}$ that is the same as that induced by the inclusion of simplicial complexes $K_{I} \longrightarrow K$;

(b) the projection $\prod_{i=1}^{m} X_{i} \longrightarrow X^{I}$ induces a map $(\underline{X}, \underline{A})^{K} \longrightarrow(\underline{X}, \underline{A})^{K_{I}}$

(c) the composite $(\underline{X}, \underline{A})^{K_{I}} \longrightarrow(\underline{X}, \underline{A})^{K} \longrightarrow(\underline{X}, \underline{A})^{K_{I}}$ is the identity map. 
Proof: Let $\bar{f}: I \longrightarrow[m]$ be the inclusion. Then $\bar{f}$ induces a map of products $\tilde{f}: X^{I} \longrightarrow \prod_{i=1}^{m} X_{i}$ by sending the $i^{\text {th }}$-factor of $X^{I}$ to the $\bar{f}(i)$-factor of $\prod_{i=1}^{m} X_{i}$. For $\sigma \in K_{I}$, write $\sigma_{K_{I}}, \sigma_{K}$ for $\sigma$ viewed as a face of $K_{I}$ or $K$ respectively. Write $|I|$ for the cardinality of $I$. Define a map of products by

$$
(\underline{X}, \underline{A})^{\sigma_{K_{I}}}=\prod_{i=1}^{|I|} Y_{i} \longrightarrow \prod_{i=1}^{|I|} Y_{f(i)} \hookrightarrow \prod_{j=1}^{m} Y_{j}=(\underline{X}, \underline{A})^{\sigma_{K}} .
$$

Taking the union over all faces in $K_{I}$, then exactly as in the proof of Proposition 3.1 (b), we obtain a map of polyhedral products

$$
(\underline{X}, \underline{A})^{K_{I}} \longrightarrow(\underline{X}, \underline{A})^{K} \text {. }
$$

Notice that this is exactly the same construction as in the proof of Proposition 3.1 (b), so the map just produced is the same as that induced by the inclusion of simplicial complexes $K_{I} \longrightarrow K$.

Next, for $I \subseteq[m]$ there is a projection

$$
\prod_{i=1}^{m} X_{i} \longrightarrow \prod_{j=1}^{|I|} X_{j}
$$

For any subspace $(\underline{X}, \underline{A})^{\sigma}=\prod_{i=1}^{m} Y_{i}$ of $\prod_{i=1}^{m} X_{i}$, we obtain a projection onto $\prod_{j=1}^{|I|} Y_{j}$. Notice that if $\sigma$ is a face of $K_{I}$ then $\prod_{j=1}^{|I|} Y_{i}=(\underline{X}, \underline{A})^{\sigma_{K_{I}}}$, and if $\sigma$ is not a face of $K_{I}$ then $\tau=\sigma \cap K_{I}$ is a face of $K_{I}$, so the projection induces a map from $(\underline{X}, \underline{A})^{\sigma_{K}}$ to $(\underline{X}, \underline{A})^{\tau}$. Taking the union over all the faces in $K$ we obtain a map

$$
(\underline{X}, \underline{A})^{K} \longrightarrow(\underline{X}, \underline{A})^{K_{I}} .
$$

Finally, as the composite $X^{I} \longrightarrow \prod_{i=1}^{m} X_{i} \longrightarrow X^{I}$ is the identity map, the constructions of the maps in the composite $(\underline{X}, \underline{A})^{\sigma_{K_{I}}} \longrightarrow$ $(\underline{X}, \underline{A})^{\sigma_{K}} \longrightarrow(\underline{X}, \underline{A})^{\sigma_{K_{I}}}$ imply that this composite is the identity map. Taking the union over all $\sigma \in K_{I}$ shows that the composite $(\underline{X}, \underline{A})^{K_{I}} \longrightarrow$ $(\underline{X}, \underline{A})^{K} \longrightarrow(\underline{X}, \underline{A})^{K_{I}}$ is the identity map.

Proposition 3.6 is often very valuable in helping to determine features of the homotopy types of polyhedral products.

Example 3.7: Return to Example 3.5. The full subcomplex $I_{3}$ of $K$ consists of two vertices. So by Example $2.2,(\underline{C X}, \underline{X})^{I_{3}} \simeq X_{3} * X_{4}$. Therefore, by Proposition 3.6 (c), $X_{3} * X_{4}$ retracts off of $(\underline{C X}, \underline{X})^{K}$. In particular, in the case of the moment-angle complex $\mathcal{Z}_{K}$, we have $X_{3}=X_{4}=\Omega \mathbb{C} P^{\infty} \simeq S^{1}$, so we obtain a retraction of $S^{3}$ off of $\mathcal{Z}_{K}$. 


\subsection{Pushouts of simplicial complexes induce pushouts of polyhedral products}

Let $K$ be a simplicial complex on the vertex set $[m]$ and suppose that there is a pushout of simplicial complexes

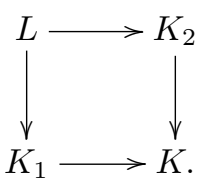

That is, $K=K_{1} \cup_{L} K_{2}$. To compare the polyhedral products for $L, K_{1}$, $K_{2}$ and $K$ we should really consider each of the four simplicial complexes as being on the same vertex set $[m]$. This may introduce "ghost" vertices if, for example, $L$ has fewer vertices than $K$. Let $\bar{L}, \bar{K}_{1}$ and $\bar{K}_{2}$ be the simplicial complexes $L, K_{1}$ and $K_{2}$, regarded as simplicial complexes on the vertex set $[m]$. Then $K=\bar{K}_{1} \cup_{\bar{L}} \bar{K}_{2}$.

Proposition 3.8: Let $K$ be a simplicial complex on the vertex set $[m]$. Suppose that there is a pushout of simplicial complexes

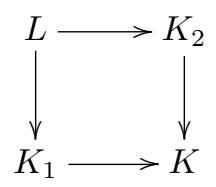

Then there is a pushout of polyhedral products

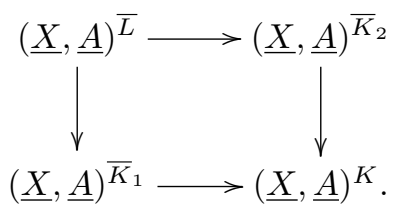

Proof: Since $K=K_{1} \cup_{L} K_{2}$ and $K$ is finite, the simplices in $K$ can be put into three finite collections: $(\mathcal{A})$ the simplices in $L,(\mathcal{B})$ the simplices in $K_{1}$ that are not simplices of $L$ and $(\mathcal{C})$ the simplices of $K_{2}$ that are not 
simplices of $L$. Then we have

$$
\begin{aligned}
L & =\bigcup_{\sigma \in \mathcal{A}} \sigma \\
K_{1} & =\left(\bigcup_{\sigma \in \mathcal{A}} \sigma\right) \cup\left(\bigcup_{\sigma^{\prime} \in \mathcal{B}} \sigma^{\prime}\right) \\
K_{2} & =\left(\bigcup_{\sigma \in \mathcal{A}} \sigma\right) \cup\left(\bigcup_{\sigma^{\prime \prime} \in \mathcal{C}} \sigma^{\prime \prime}\right) \\
K & =\left(\bigcup_{\sigma \in \mathcal{A}} \sigma\right) \cup\left(\bigcup_{\sigma^{\prime} \in \mathcal{B}} \sigma^{\prime}\right) \cup\left(\bigcup_{\sigma^{\prime \prime} \in \mathcal{C}} \sigma^{\prime \prime}\right) .
\end{aligned}
$$

By definition, for any simplicial complex $M$ on the vertex set $[m]$, $(\underline{X}, \underline{A})^{M}=\bigcup_{\sigma \in M}(\underline{X}, \underline{A})^{\sigma}$. So in our case, we have

$$
\begin{aligned}
(\underline{X}, \underline{A})^{\bar{L}} & =\bigcup_{\sigma \in \mathcal{A}}(\underline{X}, \underline{A})^{\sigma} \\
(\underline{X}, \underline{A})^{\bar{K}_{1}} & =\left(\bigcup_{\sigma \in \mathcal{A}}(\underline{X}, \underline{A})^{\sigma}\right) \cup\left(\bigcup_{\sigma^{\prime} \in \mathcal{B}}(\underline{X}, \underline{A})^{\sigma^{\prime}}\right) \\
(\underline{X}, \underline{A})^{\bar{K}_{2}} & =\left(\bigcup_{\sigma \in \mathcal{A}}(\underline{X}, \underline{A})^{\sigma}\right) \cup\left(\bigcup_{\sigma^{\prime \prime} \in \mathcal{C}}(\underline{X}, \underline{A})^{\sigma^{\prime \prime}}\right) \\
(\underline{X}, \underline{A})^{K} & =\left(\bigcup_{\sigma \in \mathcal{A}}(\underline{X}, \underline{A})^{\sigma}\right) \cup\left(\bigcup_{\sigma^{\prime} \in \mathcal{B}}(\underline{X}, \underline{A})^{\sigma^{\prime}}\right) \cup\left(\bigcup_{\sigma^{\prime \prime} \in \mathcal{C}}(\underline{X}, \underline{A})^{\sigma^{\prime \prime}}\right) .
\end{aligned}
$$

In particular, since $(\underline{X}, \underline{A})^{\bar{L}}=(\underline{X}, \underline{A})^{\bar{K}_{1}} \cap(\underline{X}, \underline{A})^{\bar{K}_{2}}$ we have

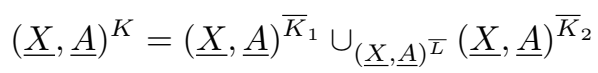

which implies the existence of the asserted pushout.

Example 3.9: Let $L=\emptyset$, and let $K_{1}=\{1\}$ and $K_{2}=\{2\}$ be single points. Then $K=K_{1} \cup_{L} K_{2}$ is two disjoint points. By definition of the polyhedral product, $(\underline{X}, \underline{A})^{\bar{L}}=A_{1} \times A_{2},(\underline{X}, \underline{A})^{\bar{K}_{1}}=X_{1} \times A_{2},(\underline{X}, \underline{A})^{\bar{K}_{2}}=A_{1} \times X_{2}$, and $(\underline{X}, \underline{A})^{K}=X_{1} \times A_{2} \cup_{A_{1} \times A_{2}} A_{1} \times X_{2}$. So $(\underline{X}, \underline{A})^{K}$ is the pushout of $(\underline{X}, \underline{A})^{\bar{K}_{1}}$ and $(\underline{X}, \underline{A})^{\bar{K}_{2}}$ over $(\underline{X}, \underline{A})^{\bar{L}}$. Note that this is exactly the same as Example 2.2.

\subsection{Fibrations and homotopy fibrations}

The next fundamental property is how the polyhedral product behaves with respect to fibrations of pairs. Proposition 3.10 was proved in [11] in the 
special case of $m$ copies of the same pairs, and stated in the more general case of $m$ different pairs in [1].

Proposition 3.10: Let $K$ be a simplicial complex on the vertex set $[\mathrm{m}]$. Suppose that for $1 \leq i \leq m$ there are maps of pairs $p_{i}:\left(E_{i}, E_{i}^{\prime}\right) \longrightarrow\left(B_{i}, B_{i}\right)$ such that the restrictions $E_{i} \longrightarrow B_{i}$ and $E_{i}^{\prime} \longrightarrow B_{i}$ are fibrations with fibres $F_{i}$ and $F_{i}^{\prime}$ respectively. Then there is a fibration

$$
\left(\underline{F}, \underline{F^{\prime}}\right)^{K} \longrightarrow\left(\underline{E}, \underline{E^{\prime}}\right)^{K} \longrightarrow \prod_{i=1}^{m} B_{i} .
$$

Proof: By Proposition 3.1 (a), the maps of pairs $\left(E_{i}, E_{i}^{\prime}\right) \longrightarrow\left(F_{i}, F_{i}^{\prime}\right)$ induce a map of polyhedral products $f:\left(\underline{E}, \underline{E^{\prime}}\right)^{K} \longrightarrow(\underline{B}, \underline{B})^{K}$. The definition of the polyhedral product implies that $(\underline{B}, \underline{B})^{K}=B_{1} \times \cdots \times B_{m}$.

First consider the fibre $F$ of $f$, that is, the collection of points in $\left(\underline{E}, \underline{E^{\prime}}\right)^{K}$ that are sent to the basepoint $(*, \ldots, *) \in B_{1} \times \cdots \times B_{m}$. Notice that for any $\sigma \in K, f$ restricts to a product map

$$
f^{\sigma}:\left(\underline{E}, \underline{E^{\prime}}\right)^{\sigma}=\prod_{i=1}^{m} Y_{i} \longrightarrow \prod_{i=1}^{m} B_{i}=(\underline{B}, \underline{B})^{\sigma}
$$

where $Y_{i}=E_{i}$ if $i \in K$ and $Y_{i}=E_{i}^{\prime}$ if $i \notin K$. Thus the fibre of $f^{\sigma}$ is the product of the fibres of the maps in the $m$ coordinates. That is, the fibre is $\prod_{i=1}^{m} Z_{i}$ where $Z_{i}=F_{i}$ if $i \in \sigma$ and $Z_{i}=F_{i}^{\prime}$ if $i \notin \sigma$. In other words, the fibre is exactly $\left(\underline{F}, \underline{F^{\prime}}\right)^{\sigma}$. Now for the fibre of $f$ as a whole we have:

$$
\begin{aligned}
F & =\left\{\left(x_{1}, \ldots, x_{m}\right) \in\left(\underline{E}, \underline{E^{\prime}}\right)^{K} \mid f\left(x_{1}, \ldots, x_{m}\right)=(*, \ldots, *)\right\} \\
& =\left\{\left(x_{1}, \ldots, x_{m}\right) \in \bigcup_{\sigma \in K}\left(\underline{E}, \underline{E^{\prime}}\right)^{\sigma} \mid f\left(x_{1}, \ldots, x_{m}\right)=(*, \ldots, *)\right\} \\
& =\bigcup_{\sigma \in K}\left\{\left(x_{1}, \ldots, x_{m}\right) \in\left(\underline{E}, \underline{E^{\prime}}\right)^{\sigma} \mid f\left(x_{1}, \ldots, x_{m}\right)=(*, \ldots, *)\right\} \\
& =\bigcup_{\sigma \in K}\left(\underline{F}, \underline{F^{\prime}}\right)^{\sigma} \\
& =\left(\underline{F}, \underline{F^{\prime}}\right)^{K} .
\end{aligned}
$$

It remains to show that the map $\left(\underline{E}, \underline{E^{\prime}}\right)^{K} \longrightarrow B_{1} \times \cdots \times B_{m}$ has the homotopy lifting property. But each of the pairs $\left(E_{i}, E_{i}^{\prime}\right) \longrightarrow\left(B_{i}, B_{i}\right)$ is a fibration and so has the homotopy lifting property. A product of fibrations is a fibration, so the map $\left(\underline{E}, \underline{E^{\prime}}\right)^{\sigma} \stackrel{f^{\sigma}}{\longrightarrow} B_{1} \times \cdots \times B_{m}$ has the homotopy lifting property. Finally, since the map $\left(\underline{E}, \underline{E^{\prime}}\right)^{K} \longrightarrow B_{1} \times \cdots \times B_{m}$ is the union of the maps $f^{\sigma}$, it too has the homotopy lifting property. 
We will now use Proposition 3.10 to produce explicit fibrations between polyhedral products. The argument roughly follows [1]. For a pointed, pathconnected space $X$ let $P X$ be the path space of $X$. That is, if $x_{0}$ is the basepoint of $X$ and 0 is the basepoint of the unit interval [0,1], then $P X$ is the space of all pointed, continuous maps $f:[0,1] \longrightarrow X$ such that $f(0)=x_{0}$. The evaluation map $e_{1}: P X \longrightarrow X$ is defined by $e_{1}(f)=f(1)$. It is well known that there is a fibration

$$
\Omega X \longrightarrow P X \stackrel{e_{1}}{\longrightarrow} X .
$$

Consider the pair $(X \times P X, P X)$ where $P X$ is the subspace of $X \times P X$ given by pairs $(f(1), f)$. Observe that there is a fibration of pairs

$$
(P X, \Omega X) \longrightarrow(X \times P X, P X) \stackrel{\pi_{X} \times e_{1}}{\longrightarrow}(X \times X)
$$

where $\pi_{X}$ is the projection. Proposition 3.10 therefore implies the following.

Proposition 3.11: Let $K$ be a simplicial complex on the vertex set $[m]$ and let $X_{1}, \ldots, X_{m}$ be pointed, path-connected spaces. Then there is a fibration

$$
(\underline{P X}, \underline{\Omega X})^{K} \longrightarrow(\underline{X \times P X}, \underline{P X})^{K} \stackrel{\left(\pi_{X} \times e_{1}\right)^{K}}{\longrightarrow} \prod_{i=1}^{m} X_{i}
$$

Next, observe that there is an equivalence of pairs

$$
(X, *) \stackrel{(\kappa, *)}{\longrightarrow}(X \times P X, P X)
$$

where $\kappa$ is defined by $\kappa(x)=\left(x, f_{x}\right)$ for $f_{x}:[0,1] \longrightarrow X$ the constant map $f_{x}(t)=x$ for all $t \in[0,1]$. Consequently, for any simplicial complex $K$, there is a homotopy equivalence $(\underline{X}, \underline{*})^{K} \simeq(\underline{X \times P X}, \underline{P X})^{K}$. Thus from the fibration in Proposition 3.11 we obtain the following.

Corollary 3.12: Let $K$ be a simplicial complex on the vertex set $[m]$ and let $X_{1}, \ldots, X_{m}$ be pointed, path-connected spaces. Then there is a homotopy fibration

$$
(\underline{P X}, \underline{\Omega X})^{K} \longrightarrow(\underline{X}, \underline{*})^{K} \longrightarrow \prod_{i=1}^{m} X_{i}
$$

Example 3.13: Take each $X_{i}=\mathbb{C} P^{\infty}$. Then $(\underline{X}, \underline{*})^{K}=D J_{K}$, implying that $(\underline{P X}, \underline{X})^{K} \simeq \mathcal{Z}_{K}$. 
Finally, it is common to replace path spaces with cones. For a pointed, path-connected space $X$, let $C X$ be the reduced cone on $X$, defined by

$$
C X=(X \times I) / \sim
$$

where $(x, 1) \sim\left(x^{\prime}, 1\right)$ for all $x, x^{\prime} \in X$ and $\left(x_{0}, t\right) \sim\left(x_{0}, 0\right)$ for all $t \in[0,1]$. Observe that there is a homotopy equivalence of pairs $(C P X, \Omega X) \longrightarrow$ $(P X, \Omega X)$ given by dilating the cone on $P X$. Notice that the inclusion of $\Omega X$ into $C P X$ factors through $C \Omega X$, and the dilation of the cone induces a homotopy equivalence of pairs $(C \Omega X, \Omega X) \longrightarrow(C P X, \Omega X)$. Thus there is a homotopy equivalence of pairs $(C \Omega X, \Omega X) \longrightarrow(P X, \Omega X)$. So Corollary 3.12 can be rewritten as follows.

Corollary 3.14: Let $K$ be a simplicial complex on the vertex set $[m]$ and let $X_{1}, \ldots, X_{m}$ be pointed, path-connected spaces. Then there is a homotopy fibration

$$
(\underline{C \Omega X}, \underline{\Omega X})^{K} \longrightarrow(\underline{X}, \underline{*})^{K} \longrightarrow \prod_{i=1}^{m} X_{i}
$$

Example 3.15: Including the wedge into the product, there is a homotopy fibration

$$
F_{m} \longrightarrow \bigvee_{i=1}^{m} X_{i} \longrightarrow \prod_{i=1}^{m} X_{i}
$$

which defines the space $F_{m}$. This homotopy fibration can be modelled by polyhedral products. Let $K$ be $m$ disjoint points. By Example 2.1, $(\underline{X}, \underline{*})^{K}=\bigvee_{i=1}^{m} X_{i}$, so the inclusion of the wedge into the product is modelled by the map $(\underline{X}, \underline{*})^{K} \longrightarrow \prod_{i=1}^{m} X_{i}$. By Corollary 3.14, the homotopy fibre of this inclusion is $(\underline{C \Omega X}, \underline{\Omega X})^{K}$. That is, $F_{m} \simeq(\underline{C \Omega X}, \underline{\Omega X})^{K}$. In Proposition 7.1, the homotopy type of $(\underline{C \Omega X}, \underline{\Omega X})^{K}$ will be determined.

\subsection{An elementary loop space decomposition}

Let $i$ be a vertex in $K$. Including $\{i\}$ into $K$ gives a map of simplicial complexes $\{i\} \longrightarrow K$. This induces a map of polyhedral products $(\underline{X}, \underline{*})^{\{i\}} \longrightarrow$ $(\underline{X}, \underline{*})^{K}$. By the definition of the polyhedral product, $(\underline{X}, \underline{*})^{K_{\{i\}}}=X_{i}$. So the previous map of polyhedral products is just the inclusion of the $i^{t h}$ coordinate space, $X_{i} \longrightarrow(\underline{X}, \underline{*})^{K}$. Therefore, when included into $\prod_{i=1}^{m} X_{i}$ we obtain the inclusion of the $i^{t h}$ factor. In general, $(\underline{X}, \underline{*})^{K}$ is not an 
$H$-space so we cannot take the product of these coordinate inclusions. But after looping there is a product, so multiplying together the maps $\Omega X_{i} \longrightarrow \Omega(\underline{X}, \underline{*})^{K}$ for $1 \leq i \leq m$ we obtain a right homotopy inverse for the map $\Omega(\underline{X}, \underline{*})^{K} \longrightarrow \prod_{i=1}^{m} \Omega X_{i}$, implying the following.

Lemma 3.16: The homotopy fibration in Corollary 3.14 splits after looping, resulting in a homotopy equivalence

$$
\Omega(\underline{X}, \underline{*})^{K} \simeq\left(\prod_{i=1}^{m} \Omega X_{i}\right) \times \Omega(\underline{C \Omega X}, \underline{\Omega X})^{K} .
$$

Example 3.17: Let $K$ be two disjoint points. By Example $2.1,(\underline{X}, \underline{*})^{K}=$ $X_{1} \vee X_{2}$, and by Example 2.2, $(\underline{C \Omega X}, \underline{\Omega X})^{K} \simeq \Omega X_{1} * \Omega X_{2}$. In this case Lemma 3.16 recovers a result of Ganea [14],

$$
\Omega\left(X_{1} \vee X_{2}\right) \simeq \Omega X_{1} \times \Omega X_{2} \times \Omega\left(\Omega X_{1} * \Omega X_{2}\right) .
$$

An important special case is for Davis-Januszkiewicz spaces and moment-angle complexes. Noting that $\Omega \mathbb{C} P^{\infty} \simeq S^{1}$, Example 3.13 and Lemma 3.16 combine to immediately give the following.

Corollary 3.18: Let $K$ be a simplicial complex on the vertex set $[m]$. Then the homotopy fibration $\mathcal{Z}_{K} \longrightarrow D J_{K} \longrightarrow \prod_{i=1}^{m} \mathbb{C} P^{\infty}$ splits after looping, giving a homotopy equivalence

$$
\Omega D J_{K} \simeq\left(\prod_{i=1}^{m} S^{1}\right) \times \Omega \mathcal{Z}_{K} .
$$

\section{The homotopy type of $\Sigma(\underline{X}, \underline{A})^{K}$}

This section considers the homotopy type of $\Sigma(\underline{X}, \underline{A})^{K}$. It turns out that this decomposes very neatly as a wedge of summands. The basic idea is to generalize the wedge decomposition for $\Sigma(X \times Y)$. Recall the following.

Lemma 4.1: Let $X$ and $Y$ be path-connected, pointed $C W$-complexes. Then there is a homotopy equivalence

$$
\Sigma(X \times Y) \simeq \Sigma X \vee \Sigma Y \vee(\Sigma X \wedge Y) .
$$


Proof: Including the wedge into the product there is a cofibration

$$
X \vee Y \longrightarrow X \times Y \longrightarrow X \wedge Y \text {. }
$$

Notice that the composite $X \vee Y \longrightarrow X \times Y \stackrel{\text { proj }}{\longrightarrow} X$ equals the pinch map onto the left wedge summand, and $X \vee Y \longrightarrow X \times Y \stackrel{\text { proj }}{\longrightarrow} Y$ is the pinch map onto the right wedge summand. As $X \times Y$ is not a co- $H$-space, the two projections cannot be added to produce a left homotopy inverse for the inclusion $X \vee Y \longrightarrow X \times Y$, but after suspending this can be done. Thus the homotopy cofibration $\Sigma X \vee \Sigma Y \longrightarrow \Sigma(X \times Y) \longrightarrow \Sigma X \wedge Y$ splits to give a homotopy equivalence $\Sigma(X \times Y) \simeq \Sigma X \vee \Sigma Y \vee(\Sigma X \wedge Y)$.

Iterating gives the following.

Lemma 4.2: Let $X_{1}, \ldots, X_{m}$ be path-connected, pointed $C W$-complexes. Then there is a homotopy equivalence

$$
\Sigma\left(X_{1} \times \cdots \times X_{m}\right) \simeq \bigvee_{1 \leq i_{1}<\cdots i_{k} \leq m} \Sigma X_{i_{1}} \wedge \cdots \wedge X_{i_{k}} .
$$

In terms of polyhedral products, $X_{1} \times \cdots \times X_{m}=(\underline{X}, \underline{A})^{\Delta^{m-1}}$. Notice that the faces of $\Delta^{m-1}$ are in one-to-one correspondence with the sequences $\left(i_{1}, \ldots, i_{k}\right)$ for $1 \leq i_{1}<\cdots<i_{k} \leq m$, and each such face corresponds to the polyhedral product $X_{i_{1}} \times \cdots \times X_{i_{k}}$. Thus, if $\sigma=\left(i_{1}, \ldots, i_{k}\right)$ is a face of $K$ and we write

$$
\underline{X}^{\wedge \sigma}=X_{i_{1}} \wedge \cdots \wedge X_{i_{k}},
$$

then the homotopy equivalence in Lemma 4.2 can be rewritten as

$$
\Sigma\left(X_{1} \times \cdots \times X_{m}\right) \simeq \bigvee_{\sigma \in K} \Sigma \underline{X}^{\wedge \sigma} .
$$

Therefore it could be expected that, at least in the case $(\underline{X}, \underline{*})^{K}$, we should have $\Sigma(\underline{X}, \underline{*})^{K}$ consisting of those wedge summands in $\Sigma\left(X_{1} \times \cdots \times X_{m}\right)$ that correspond to the faces of $K$. In fact, this is exactly what happens.

Proposition 4.3: Let $K$ be a simplicial complex on the vertex set $[m]$ and let $X_{1}, \ldots, X_{m}$ be path-connected, pointed $C W$-complexes. Then there is a homotopy equivalence

$$
\Sigma(\underline{X}, \underline{*})^{K} \simeq \bigvee_{\sigma \in K} \Sigma \underline{X}^{\wedge \sigma}
$$


Proof: Let $\sigma=\left(i_{1}, \ldots, i_{k}\right)$ be a face of $K$. By definition of the polyhedral product

$$
(\underline{X}, \underline{*})^{\sigma}=\prod_{i=1}^{m} Y_{i}
$$

where $Y_{i}=X_{i}$ if $i \in \sigma$ and $Y_{i}=*$ if $i \notin \sigma$. That is,

$$
(\underline{X}, \underline{*})^{\sigma}=\prod_{j=1}^{k} X_{i_{j}} .
$$

Therefore, by (4.1) there is a homotopy equivalence

$$
\Sigma(\underline{X}, \underline{*})^{\sigma} \simeq \bigvee_{\tau \in \sigma} \Sigma \underline{X}^{\wedge \tau}
$$

On the other hand, observe that any proper sub-product of $(\underline{X}, \underline{*})^{\sigma}=$ $\prod_{j=1}^{k} X_{i_{j}}$ is the polyhedral product of a corresponding proper sub-face of $K$. Write $\partial \sigma$ for the union of proper sub-faces of $\sigma$. Then there is an identification of quotient spaces

$$
\begin{aligned}
(\underline{X}, \underline{*})^{\sigma} /(\underline{X}, \underline{*})^{\partial \sigma} & =\left(\prod_{j=1}^{k} X_{i_{j}}\right) /(\text { union of proper sub-products }) \\
& =X_{i_{1}} \wedge \cdots \wedge X_{i_{k}} .
\end{aligned}
$$

By Lemma 4.2 , the quotient map $(\underline{X}, \underline{*})^{\sigma}=X_{i_{1}} \times \cdots \times X_{i_{k}} \longrightarrow X_{i_{1}} \wedge \cdots \wedge X_{i_{k}}$ has a right homotopy inverse after suspending. Thus there is a homotopy equivalence

$$
\Sigma(\underline{X}, \underline{*})^{\sigma} \simeq \Sigma(\underline{X}, \underline{*})^{\partial \sigma} \vee\left(\Sigma X_{i_{1}} \wedge \cdots \wedge X_{i_{k}}\right)=\Sigma(\underline{X}, \underline{*})^{\partial \sigma} \vee \Sigma \underline{X}^{\wedge \sigma} .
$$

Comparing the two homotopy equivalences for $\Sigma(\underline{X}, \underline{*})^{\sigma}$ we obtain

$$
\Sigma(\underline{X}, \underline{*})^{\partial \sigma} \simeq \bigvee_{\tau \in \partial \sigma} \Sigma \underline{X}^{\wedge \tau}
$$


Now consider the string of equivalences

$$
\begin{aligned}
\Sigma(\underline{X}, \underline{*})^{K} & =\Sigma\left(\bigcup_{\sigma \in K}(\underline{X}, \underline{*})^{\sigma}\right) \\
& \simeq \bigcup_{\sigma \in K} \Sigma(\underline{X}, \underline{*})^{\sigma} \\
& \simeq \bigcup_{\sigma \in K}\left(\Sigma(\underline{X}, \underline{*})^{\partial \sigma} \vee\left(\Sigma X_{i_{1}} \wedge \cdots \wedge X_{i_{k}}\right)\right) \\
& =\bigcup_{\sigma \in K} \Sigma X_{i_{1}} \wedge \cdots \wedge X_{i_{k}} \\
& \simeq \bigvee_{\sigma \in K} \Sigma X_{i_{1}} \wedge \cdots \wedge X_{i_{k}}
\end{aligned}
$$

The first equality holds by definition of the polyhedral product, the second holds because the reduced suspension over a union is homotopy equvalent to the union of the reduced suspensions over each term in the union, the third holds by $(4.2)$, the fourth holds because the decomposition for $\Sigma(\underline{X}, \underline{*})^{\partial \sigma}$ in (4.3) is in terms of proper sub-faces of $\sigma$ which are otherwise accounted for in the union, and the fifth holds because the terms in the fourth line intersect only at the basepoint. The string of equivalences as a whole gives the asserted homotopy equivalence.

Example 4.4: Let $K$ be the following simplical complex on 4 vertices:

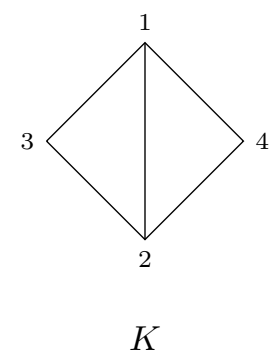

The faces of $K$ are the vertices $\{1\},\{2\},\{3\},\{4\}$ and the edges $(1,2)$, $(1,3),(1,4),(2,3)$ and $(2,4)$. For a vertex $\{i\}$ we have $\underline{X}^{\{i\}}=X_{i}$ and for an edge $(i, j)$ we have $\underline{X}^{(i, j)}=X_{i} \wedge X_{j}$. So by Proposition 4.3 there is a homotopy equivalence

$$
\begin{aligned}
&(\underline{X}, \underline{*})^{K} \simeq \Sigma X_{1} \vee \Sigma X_{2} \vee \Sigma X_{3} \vee \Sigma X_{4} \vee\left(\Sigma X_{1} \wedge X_{2}\right) \vee \\
&\left(\Sigma X_{1} \wedge X_{3}\right) \vee\left(\Sigma X_{1} \wedge X_{4}\right) \vee\left(\Sigma X_{2} \wedge X_{3}\right) \vee\left(\Sigma X_{2} \wedge X_{4}\right) .
\end{aligned}
$$


Proving a decomposition for $\Sigma(\underline{X}, \underline{A})^{K}$ in the general case is a bit more involved because the subspaces $A_{i}$ of $X_{i}$ need not be points. But the idea behind the argument remains the same. Instead of proving the general statement we will just state it. This requires some notation.

Let $K$ be a simplicial complex on the vertex set $[m]$ and for $1 \leq i \leq m$ let $\left(X_{i}, A_{i}\right)$ be pairs of pointed, path-connected $C W$-complexes. Recall that

$$
(\underline{X}, \underline{A})^{\sigma}=\prod_{i=1}^{m} Y_{i} \quad \text { where } \quad Y_{i}=\left\{\begin{array}{l}
X_{i} \text { if } i \in K \\
A_{i} \text { if } i \notin K
\end{array}\right.
$$

and the polyhedral product is

$$
(\underline{X}, \underline{A})^{K}=\bigcup_{\sigma \in K}(\underline{X}, \underline{A})^{\sigma} .
$$

For $\sigma \in K$, let

$$
\widehat{(\underline{X}, \underline{A}}^{\sigma}=\bigwedge_{i=1}^{m} Y_{i} \quad \text { where } \quad Y_{i}=\left\{\begin{array}{l}
X_{i} \text { if } i \in K \\
A_{i} \text { if } i \notin K
\end{array}\right.
$$

The smash polyhedral product is

$$
\widehat{(\underline{X}, \underline{A})}^{K}=\bigcup_{\sigma \in K} \widehat{(\underline{X}, \underline{A}}^{\sigma} .
$$

Another way to think of this is to recall that $(\underline{X}, \underline{A})^{K}$ is a subspace of the product $\prod_{i=1}^{m} X_{i}$, and $\widehat{(\underline{X}, \underline{A}}^{K}$ is the image of $(\underline{X}, \underline{A})^{K}$ under the natural quotient map $\prod_{i=1}^{m} X_{i} \longrightarrow \bigwedge_{i=1}^{m} X_{i}$.

Let $I \subset[m]$. By Proposition 3.6, there is a map of polyhedral products $(\underline{X}, \underline{A})^{K} \longrightarrow(\underline{X}, \underline{A})^{K_{I}}$. Composing, we obtain a map into a smash polyhedral product,

$$
(\underline{X}, \underline{A})^{K} \longrightarrow(\underline{X}, \underline{A})^{K_{I}} \longrightarrow \widehat{(\underline{X}, \underline{A}}^{K_{I}} .
$$

Such a composition exists for each $I \subseteq[m]$. Suspending, these can be added, giving a composition

$$
\bar{H}: \Sigma(\underline{X}, \underline{A})^{K} \longrightarrow \bigvee_{I \subseteq[m]} \Sigma(\underline{X}, \underline{A})^{K_{I}} \longrightarrow \bigvee_{I \subseteq[m]} \Sigma \widehat{(\underline{X}, \underline{A}}^{K_{I}} .
$$

Bahri, Bendersky, Cohen and Gitler [1] proved the following.

Theorem 4.5: Let $K$ be a simplicial complex on the vertex set [ $m]$ and for $1 \leq i \leq m$ let $\left(X_{i}, A_{i}\right)$ be pairs of pointed, path-connected $C W$-complexes. 
The map

$$
\bar{H}: \Sigma(\underline{X}, \underline{A})^{K} \longrightarrow \bigvee_{I \subseteq[m]} \Sigma \widehat{(\underline{X}, \underline{A}}^{K_{I}}
$$

is a homotopy equivalence.

Further, in the special case when each $X_{i}$ is contractible, Bahri, Bendersky, Cohen and Gitler show that: (i) if $I \in K$ then the smash polyhedral product $\widehat{(\underline{X}, \underline{A}}^{K_{I}}$ is contractible [1] ; and (ii) if $I \notin K$ and $I=\left(i_{1}, \ldots, i_{k}\right)$ then there is a homotopy equivalence $\widehat{(\underline{X}, \underline{A}}^{K_{I}} \simeq \Sigma\left(\left|K_{I}\right| \wedge \widehat{A}^{I}\right)$ where $\widehat{A}^{I}=\bigwedge_{j=1}^{k} A_{i_{j}}[1]$. Changing notation a bit, write the pairs as $\left(C X_{i}, X_{i}\right)$, where $C X_{i}$ is the reduced cone on $X_{i}$. Now $(\underline{\widehat{C X}, \underline{X}})^{K_{I}} \simeq \Sigma\left(\left|K_{I}\right| \wedge \widehat{X}^{I}\right)$ and we obtain the following.

Theorem 4.6: Let $K$ be a simplicial complex on the vertex set $[m]$ and for $1 \leq i \leq m$ let $X_{i}$ be a pointed, path-connected $C W$-complex. Then the map $\bar{H}$ specializes to a homotopy equivalence

$$
H: \Sigma(\underline{C X}, \underline{X})^{K} \longrightarrow \bigvee_{I \notin K} \Sigma(\widehat{\widehat{C X}, \underline{X}})^{K_{I}} \stackrel{\simeq}{\longrightarrow} \bigvee_{I \notin K} \Sigma^{2}\left(\left|K_{I}\right| \wedge \widehat{X}^{I}\right)
$$

For the moment-angle complex $\mathcal{Z}_{K}$, each pair is $\left(D^{2}, S^{1}\right)$, which is homeomorphic to the pair $\left(C S^{1}, S^{1}\right)$. As each $X_{i}=S^{1}$, we have $\widehat{X}^{I}=$ $\bigwedge_{j=1}^{k}\left(S^{1}\right)_{i_{j}} \simeq S^{k}$. If $I=\left\{i_{1}, \ldots, i_{k}\right\}$, let $|I|=k$. We obtain the following corollary.

Corollary 4.7: Let $K$ be a simplicial complex on the vertex set $[m]$. There is a homotopy equivalence

$$
\Sigma \mathcal{Z}_{K} \longrightarrow \bigvee_{I \notin K} \Sigma^{|I|+2}\left|K_{I}\right| .
$$


Example 4.8: Return to the simplicial complex $K$ in Example 4.4:

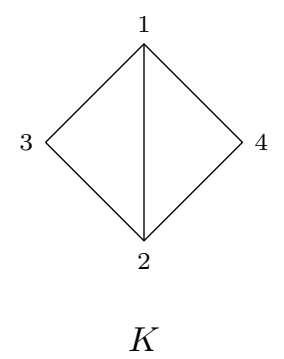

The missing faces of $K$ are $(3,4),(1,2,3),(1,2,4),(1,3,4),(2,3,4)$ and $(1,2,3,4)$. The full subcomplexes $K_{I}$ of $K$ on these vertex sets are:

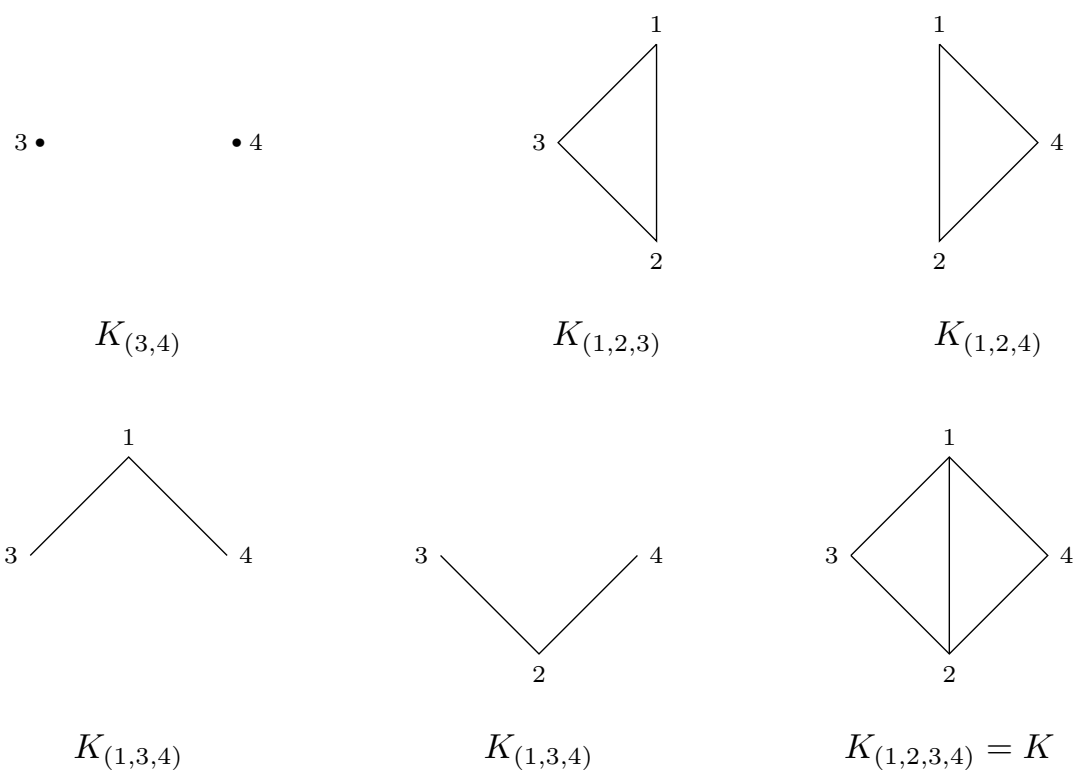

First, observe that $\left|K_{(3,4)}\right|=S^{0}$ and $|(3,4)|=2$ so $\Sigma^{|I|+2}\left|K_{I}\right|$ in this case is $S^{4}$. Second, observe that $\left|K_{(1,2,3)}\right|=S^{1}$ and $|(1,2,3)|=3$ so $\Sigma^{|I|+2}\left|K_{I}\right|$ in this case is $S^{6}$. Similarly, $K_{(1,2,4)}$ contributes an $S^{6}$ to $\Sigma \mathcal{Z}_{K}$. Third, observe that $\left|K_{(1,3,4)}\right|$ is contractible, so $\Sigma^{|I|+2}\left|K_{I}\right|$ in this case is $*$. Similarly, $K_{(2,3,4)}$ contributes a point to $\Sigma \mathcal{Z}_{K}$. Finally, observe that $\left|K_{(1,2,3,4)}\right|=S^{1} \vee S^{1}$ and $|(1,2,3,4)|=4$, so $\Sigma^{|I|+2}\left|K_{I}\right|$ in this case is $S^{7} \vee S^{7}$. Hence there is a homotopy equivalence

$$
\Sigma \mathcal{Z}_{K} \simeq S^{4} \vee S^{6} \vee S^{6} \vee S^{7} \vee S^{7} .
$$




\section{Building simplicial complexes and polyhedral products vertex by vertex}

Having determined the homotopy type of $\Sigma(\underline{X}, \underline{A})^{K}$, we now turn our attention to the homotopy type of $(\underline{X}, \underline{A})^{K}$ itself. One approach to this is to build up the simplicial complex one vertex at a time and examine the affect on the homotopy type of the corresponding polyhedral product. This approach has had good success $[17,18,19,21,22,13]$.

\subsection{Building simplicial complexes}

Let $K$ be a simplicial complex on the vertex set $[m]$. For a vertex $v \in K$, the star, restriction (or deletion) and link of $v$ are the subcomplexes

$$
\begin{aligned}
\operatorname{star}_{K}(v) & =\{\tau \in K \mid\{v\} \cup \tau \in K\} ; \\
K \backslash\{v\}=K_{[m] \backslash\{v\}} & =\{\tau \in K \mid\{v\} \cap \tau=\emptyset\} ; \\
& \operatorname{link}_{K}(v)=\operatorname{star}_{K}(v) \cap K \backslash\{v\} .
\end{aligned}
$$

The join of two simplicial complexes $K_{1}, K_{2}$ on disjoint index sets is the simplicial complex

$$
K_{1} * K_{2}=\left\{\sigma_{1} \cup \sigma_{2} \mid \sigma_{i} \in K_{i}\right\} .
$$

From the definitions, it follows that $\operatorname{star}_{K}(v)$ is a join,

$$
\operatorname{star}_{K}(v)=\{v\} * \operatorname{link}_{K}(v),
$$

and there is a pushout of simplicial complexes

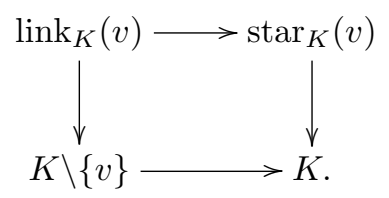

Example 5.1: Let $K$ be the simplical complex on 4 vertices:

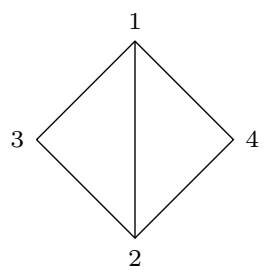


The star, restriction and link subcomplexes for the vertex 1 are:

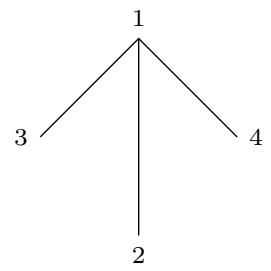

$\operatorname{star}_{K}(1)$

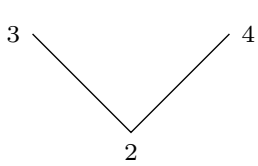

$K \backslash\{1\}$
$3 \bullet$

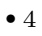

Notice that $\operatorname{star}_{K}(1)$ is clearly $\{1\} * \operatorname{link}_{K}(1)$, and $K$ is the union of $\operatorname{star}_{K}(1)$ and $K \backslash\{1\}$ over $\operatorname{link}_{K}(1)$.

A family of simplicial complexes whose polyhedral products have been studied by this vertex-by-vertex approach is shifted complexes.

Definition 5.2: A simplicial complex $K$ is shifted if there is an ordering on its vertices such that whenever $\sigma \in K, \nu$ is a vertex of $\sigma$, and $\nu^{\prime}<\nu$, then $(\sigma-\nu) \cup \nu^{\prime} \in K$.

Equivalently, regard the faces of $K$ as sequences $\left(i_{1}, \ldots, i_{k}\right)$ where $1 \leq$ $i_{1}<\cdots<i_{k} \leq m$. Order the faces by saying that $\left(j_{1}, \ldots, j_{t}\right) \leq\left(i_{1}, \ldots, i_{k}\right)$ if $t \leq k$ and $j_{1} \leq i_{1}, \cdots, j_{t} \leq i_{t}$. Then $K$ is shifted if, whenever $\sigma=$ $\left(i_{1}, \ldots, i_{k}\right) \in K$, then $\tau \in K$ for all $\tau \leq \sigma$.

Example 5.3: Consider the three simplicial complexes

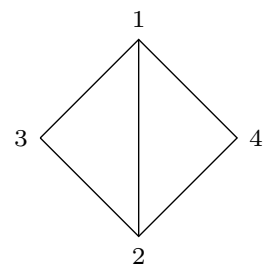

$K_{1}$

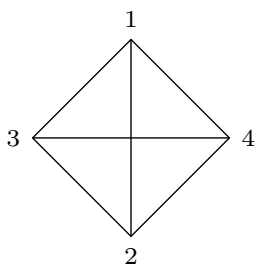

$K_{2}$

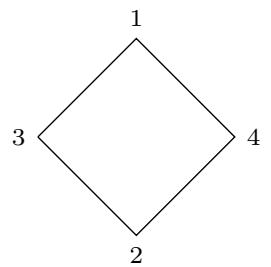

$K_{3}$

The simplicial complex $K_{1}$ is shifted using the given ordering of the vertices $(1<2<3<4)$, as is $K_{2}$. However, $K_{3}$ is not shifted under any ordering of the vertices. In the given ordering, as $(1,3) \in K_{3}$, if $K_{3}$ were shifted then $(1,2)$ would also have to be in $K_{3}$, which it is not. Under any reordering of the vertices, the vertex 4 would have to be connected to two other vertices 
by an edge: the only choices that could lead to a shifted complex are 1 and 2. But then 3 also has to be connected by an edge to 1 and 2, and now we are back to the description of $K_{3}$ with its given ordering of vertices, which is not shifted.

Another useful collection of examples is given by the full $t$-skeletons of the $(m-1)$-simplex.

Definition 5.4: Let $K$ be a simplicial complex on the vertex set $[m]$. For $0 \leq t \leq m-1$, the full $t$-skeleton of $K$ is the subcomplex $K_{t}$ consisting of those faces $\sigma \in K$ on $t+1$ vertices for $t \leq k$.

For example, the 0 -skeleton of $K$ consists of the vertices, the 1-skeleton consists of the vertices and edges, and the $(m-1)$-skeleton is all of $K$.

Example 5.5: For $0 \leq t \leq m-1$, the full $t$-skeleton $\Delta_{t}^{m-1}$ of the $(m-1)$ simplex $\Delta^{m-1}$ is shifted. The shifted property is a consequence of the fact that the symmetric group on $m$ letters acts freely on $\Delta_{t}^{m-1}$ by permuting the vertices.

\subsection{Building polyhedral products}

Let $K$ be a simplicial complex on the vertex set $[m]$. We will fix the vertex $m$ for convenience. By (5.1) there is a pushout describing $K$ as the union of $\operatorname{star}_{K}(m)$ and $K \backslash\{m\}$ over $\operatorname{link}_{K}(m)$. As for Proposition 3.8, to compare the polyhedral products for the four simplicial complexes $K, \operatorname{link}_{K}(m)$, $\operatorname{star}_{K}(m)$ and $K \backslash\{m\}$, we should really consider each simplicial complex as being on the vertex set $[m]$. This changes nothing for $K$ and $\operatorname{star}_{K}(m)$ as their vertex sets are already $[m]$ but it introduces a ghost vertex $\{m\}$ for $\operatorname{link}_{K}(m)$ and $K \backslash\{m\}$. Let $\overline{\operatorname{link}_{K}(m)}$ and $\overline{K \backslash\{m\}}$ be the simplicial complexes $\operatorname{link}_{K}(m)$ and $K \backslash\{m\}$, regarded as simplicial complexes on the vertex set $[m]$. By Proposition 3.8, the pushout of simplicial complexes in (5.1) induces a pushout of polyhedral products

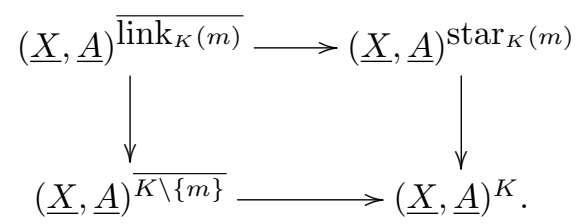

The spaces and maps in (5.2) can be better identified. First, the definition of the polyhedral product implies that

$$
(\underline{X}, \underline{A})^{\overline{\operatorname{lnk}_{K}(m)}}=(\underline{X}, \underline{A})^{\operatorname{link}_{K}(m)} \times A_{m}
$$




$$
(\underline{X}, \underline{A})^{\overline{K \backslash\{m\}}}=(\underline{X}, \underline{A})^{K \backslash\{m\}} \times A_{m} .
$$

By Proposition 3.1 (b), the inclusion of simplicial complexes $\operatorname{link}_{K}(m) \longrightarrow$ $K \backslash\{m\}$ induces a map of polyhedral products

$$
j:(\underline{X}, \underline{A})^{\operatorname{link}_{K}(m)} \longrightarrow(\underline{X}, \underline{A})^{K \backslash\{m\}} .
$$

Including the ghost vertex at $m$, the map $\overline{\operatorname{link}_{K}(m)} \longrightarrow \overline{K \backslash\{m\}}$ therefore induces the map of polyhedral products

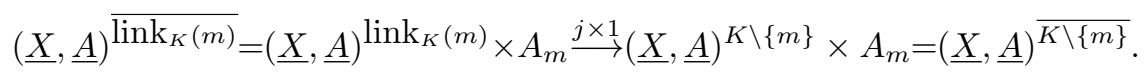

Second, the definition of the join of two simplicial complexes implies that if $K=K_{1} * K_{2}$ then there is a homeomorphism

$$
(\underline{X}, \underline{A})^{K} \cong(\underline{X}, \underline{A})^{K_{1}} \times(\underline{X}, \underline{A})^{K_{2}} .
$$

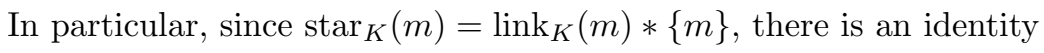

$$
(\underline{X}, \underline{A})^{\operatorname{star}_{K}(m)} \cong(\underline{X}, \underline{A})^{\operatorname{link}_{K}(m)} \times X_{m} .
$$

We may also regard $\overline{\operatorname{link}_{K}(m)}$ as $\operatorname{link}_{K}(m) * \emptyset$, where $\emptyset$ corresponds to the ghost vertex at $\{m\}$. The inclusion $\operatorname{link}_{K}(m) \longrightarrow \operatorname{star}_{K}(m)$ therefore induces a map of joins

$$
\varlimsup \operatorname{link}_{K}(m)=\operatorname{link}_{K}(m) * \emptyset \longrightarrow \operatorname{link}_{K}(m) *\{m\}=\operatorname{star}_{K}(m) .
$$

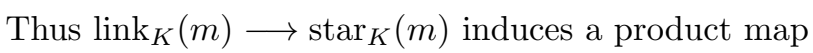

$$
(\underline{X}, \underline{A})^{\operatorname{link}_{K}(m)} \times A_{m} \stackrel{1 \times i_{m}}{\longrightarrow}(\underline{X}, \underline{A})^{\operatorname{link}_{K}(m)} \times X_{m}
$$

where $i_{m}$ is the inclusion of $A_{m}$ into $X_{m}$.

Combining these two observations, (5.2) can be rewritten as follows.

Proposition 5.6: Let $K$ be a simplicial complex on the vertex set $[m]$. Let $(\underline{X}, \underline{A})$ be a sequence of pointed $C W$-pairs $\left(X_{i}, A_{i}\right)$ for $1 \leq i \leq m$. Then there is a pushout of polyhedral products

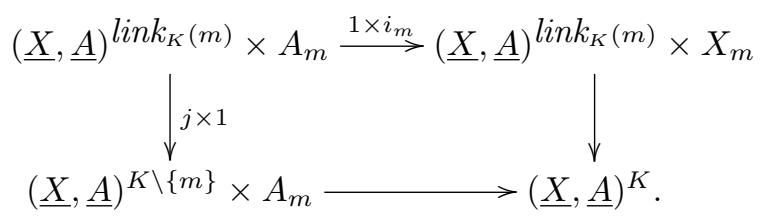


An important special case is that for $(\underline{C X}, \underline{X})$. Then the map $(\underline{C X}, \underline{X})^{\operatorname{link}_{K}(m)} \times X \stackrel{1 \times i_{m}}{\longrightarrow}(\underline{C X}, \underline{X})^{\operatorname{link}_{K}(m)} \times C X_{m}$ is equivalent, up to homotopy, to the map $(\underline{C X}, \underline{X})^{\operatorname{link}_{K}(m)} \times X_{m} \stackrel{\pi_{1}}{\longrightarrow}(\underline{C X}, \underline{X})^{\operatorname{link}_{K}(m)}$, where $\pi_{1}$ is the projection onto the first factor. Thus Proposition 5.6 specializes to the following.

Corollary 5.7: Let $K$ be a simplicial complex on the vertex set $[m]$. For $1 \leq i \leq m$ let $X_{i}$ be a pointed $C W$-complex. Then there is a homotopy pushout

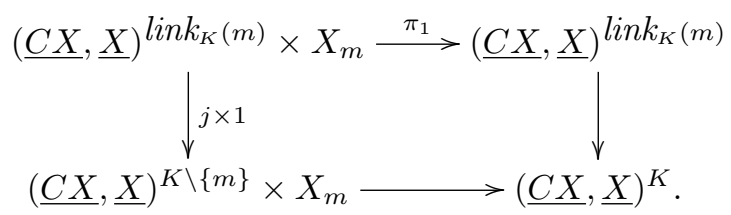

In principle, if the homotopy class of the map $j$ is known then it should be possible to identify the homotopy type of $(\underline{C X}, \underline{X})^{K}$. This is essentially the approach taken in $[18,19,21,22]$ in order to identify the homotopy type of $(\underline{C X}, \underline{X})^{K}$ when $K$ is a shifted complex. In that case, the map $j$ is null homotopic. The next section reviews the homotopy theory needed to handle the case when $j$ is null homotopic. In cases when $K$ is not shifted the map $j$ may not be null homotopic, but the same principle should apply. However, this is largely unexplored territory.

\section{Identifying the homotopy type of certain pushouts}

We begin with some definitions. Let $I$ be the unit interval. Let $A$ and $B$ be pointed topological spaces. Recall that the reduced join of $A$ and $B$ is the quotient space

$$
A * B=(A \times I \times B) / \sim
$$

where $(a, 1, b) \sim\left(a^{\prime}, 1, b\right),(a, 0, b) \sim\left(a, 0, b^{\prime}\right)$ and $(*, t, *) \sim *$ for all $a, a^{\prime} \in A, b, b^{\prime} \in B$ and $t \in I$. Ignoring the relation $(*, t, *) \sim *$ for the moment, the unreduced join can be pictured as follows:

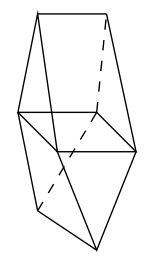


where the middle square is $A \times B$, with $B$ running horizontally. Notice that the top part of $A * B$ is $\overline{C A} \times B$, where $\overline{C A}$ is the unreduced cone on $A$, and the bottom part of $A * B$ is $A \times \overline{C B}$. These are glued together in the middle at $A \times B$. The reduced join has the same description, using the reduced cone. Thus there is a topological pushout

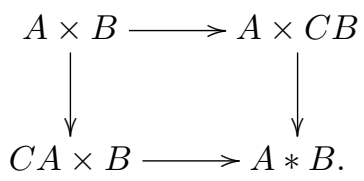

Since $C A$ and $C B$ are contractible, the maps $A \times B \longrightarrow A \times C B$ and $A \times B \longrightarrow C A \times B$ are equivalent, up to homotopy, to the projections $A \times B \stackrel{\pi_{1}}{\longrightarrow} A$ and $A \times B \stackrel{\pi_{2}}{\longrightarrow} B$ respectively. The contractibility of $C B$ also implies that the homotopy class of the map $A \times C B \longrightarrow A * B$ is determined by the restriction to $A$. The commutativity of (6.1) implies that this restriction equals the composite $A \hookrightarrow A \times B \longrightarrow C A \times B \longrightarrow A * B$. The latter is null homotopic since it factors through $C A$. Therefore the map $A \times C B \longrightarrow A * B$ is null homotopic. Similarly, the map $C A \times B \longrightarrow A * B$ is null homotopic. Putting all this together we obtain a homotopy-theoretic version of (6.1).

Lemma 6.1: There is a homotopy pushout

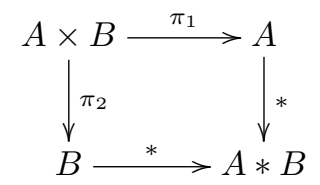

where $\pi_{1}$ and $\pi_{2}$ are the projections onto the first and second factors respectively.

The (right) half-smash of two pointed spaces $C$ and $B$ is the quotient space

$$
C \rtimes B=C \times B / \sim
$$

where $(*, b) \sim *$. In particular, if $i_{2}: B \longrightarrow C \times B$ is the inclusion of the second factor, then there is a cofibration $B \stackrel{i_{2}}{\longrightarrow} C \times B \longrightarrow C \rtimes B$. In what follows it is better to regard this cofibration as a pushout

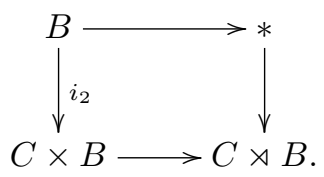


With Corollary 5.7 in mind, we now prove a key lemma that identifies the homotopy type of certain pushouts.

Lemma 6.2: Suppose that there is a homotopy pushout

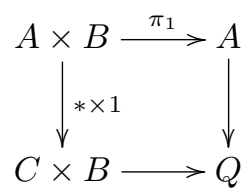

where $\pi_{1}$ is the projection. Then there is a homotopy equivalence

$$
Q \simeq(A * B) \vee(C \rtimes B)
$$

Proof: The map $A \times B \stackrel{* \times 1}{\longrightarrow} C \times B$ is equal to the composite $A \times B \stackrel{\pi_{2}}{\longrightarrow}$ $B \stackrel{i_{2}}{\longrightarrow} C \times B$. Thus, using Lemma 6.1 , the homotopy pushout in the statement of the lemma may be written as an iterated homotopy pushout diagram

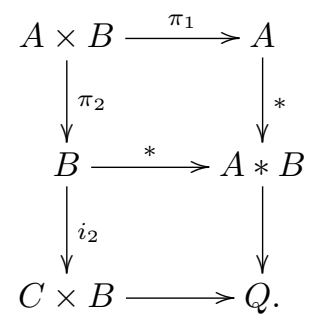

For the bottom square, since the map $B \stackrel{*}{\longrightarrow} A * B$ factors as the composite $B \longrightarrow * \longrightarrow A * B$, using (6.2) we obtain an iterated homotopy pushout diagram

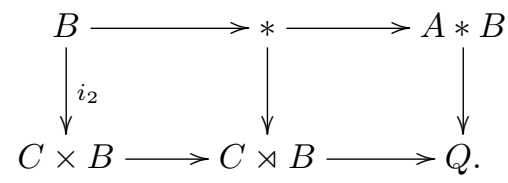

The right square immediately implies that $Q \simeq(A * B) \vee(C \rtimes B)$. 


\section{A strategy for identifying the homotopy type of $(\underline{C X}, \underline{X})^{K}$ for certain $K$}

Consider the homotopy pushout

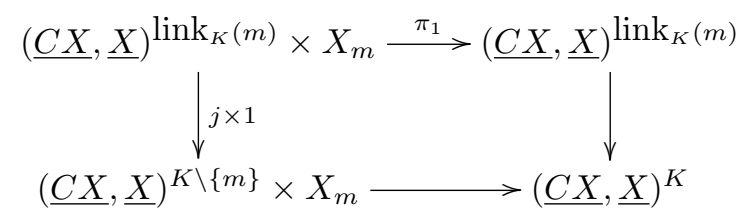

from Corollary 5.7. If the map $j$ is null homotopic then Lemma 6.2 implies that there is a homotopy equivalence

$$
(\underline{C X}, \underline{X})^{K} \simeq\left((\underline{C X}, \underline{X})^{\operatorname{link}_{K}(m)} * X_{m}\right) \vee\left((\underline{C X}, \underline{X})^{K \backslash\{m\}} \rtimes X_{m}\right) .
$$

Notice that on the right side both $\operatorname{link}_{K}(m)$ and $K \backslash\{m\}$ are simplicial complexes on the smaller vertex set $[m-1]$. This suggests an inductive procedure.

What is needed is a family $\mathcal{K}$ of simplicial complexes such that, if $K \in \mathcal{K}$ is on the vertex set $[m]$ then: (i) the vertices of $K$ may be ordered so that

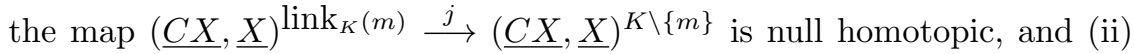
both $\operatorname{link}_{K}(m)$ and $K \backslash\{m\}$ are in $\mathcal{K}$. In particular, property (ii) implies that both $\operatorname{link}_{K}(m)$ and $K \backslash\{m\}$ have property (i), so that they also satisfy homotopy decompositions as in (7.1), and these decompositions can then be substituted into that for $(\underline{C X}, \underline{X})^{K}$. Iterating, we would obtain a fine decomposition of $(\underline{C X}, \underline{X})^{K}$.

It would be even better if an extra condition were satisfied. It is well known that if $C$ is a co- $H$-space then there is a homotopy equivalence $C \rtimes B \simeq C \vee(C \wedge B)$. So if $(\underline{C X}, \underline{X})^{K \backslash\{m\}}$ was a suspension, then (7.1) refines to a homotopy equivalence

$$
(\underline{C X}, \underline{X})^{K} \simeq\left((\underline{C X}, \underline{X})^{\operatorname{link}_{K}(m)} * X_{m}\right) \vee(\underline{C X}, \underline{X})^{K \backslash\{m\}} \vee\left((\underline{C X}, \underline{X})^{K \backslash\{m\}} \wedge X_{m}\right) .
$$

In general, there is a homotopy equivalence $A * B \simeq \Sigma A \wedge B$. So if $(\underline{C X}, \underline{X})^{K \backslash\{m\}}$ was a suspension, then not only does (7.2) hold, but it implies that $(\underline{C X}, \underline{X})^{K}$ is also a suspension. Again, as $K$ has one more vertex than $K \backslash\{m\}$, we are in the position of producing an inductive procedure.

Let's carry this through with an example to see the strategy in action. For $m \geq 1$, let $K_{m}$ be the simplicial complex consisting of $m$ disjoint vertices. There is a trivial case: as $K_{1}$ has 1 vertex, by definition of the polyhedral product $(\underline{C X}, \underline{X})^{K_{1}}=C X_{1}$ and so is contractible. 
Proposition 7.1: If $m \geq 2$ then there is a homotopy equivalence

$$
(\underline{C X}, \underline{X})^{K_{m}} \simeq \bigvee_{j=2}^{m}\left(\bigvee_{1 \leq i_{1}<\cdots<i_{j} \leq m}(j-1) \Sigma X_{i_{1}} \wedge \cdots \wedge X_{i_{j}}\right) .
$$

Proof: The proof is by induction on the number of vertices. As $K_{2}$ has 2 vertices, by Example 2.2 there is a homotopy equivalence $(\underline{C X}, \underline{X})^{K_{2}} \simeq$ $X_{1} * X_{2} \simeq \Sigma X_{1} \wedge X_{2}$, as asserted. Suppose that the homotopy equivalence in the statement of the Proposition holds for $K_{m-1}$. Consider the pushout of simplicial complexes

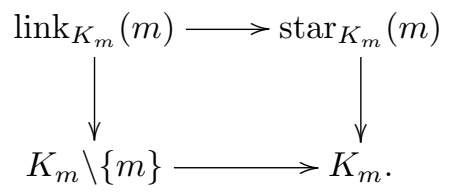

Since $K_{m}$ is $m$ disjoint vertices, $\operatorname{star}_{K_{m}}(m)=\{m\}$ and $K_{m} \backslash\{m\}=K_{m-1}$. So $\operatorname{link}_{K_{m}}(m)=\operatorname{star}_{K_{m}}(m) \cap K_{m} \backslash\{m\}=\emptyset$ and the pushout of simplicial complexes above is the pushout

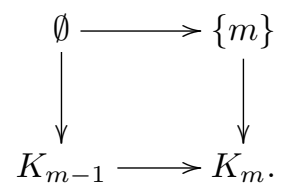

Note here that $\emptyset$ is regarded as a simplicial complex on the vertex set $[m-1]$. By Corollary 5.7 there is a homotopy pushout

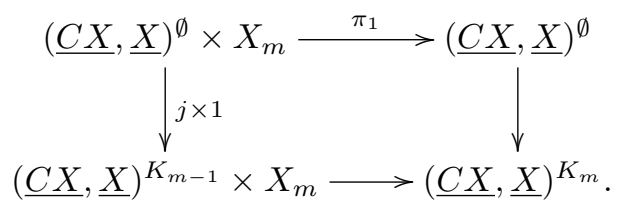

We aim to show that the map $j$ is null homotopic. For the sequence of $C W$-complexes $(\underline{C X}, \underline{X})$ the definition of the polyhedral product implies that

$$
(\underline{C X}, \underline{X})^{\emptyset}=X_{1} \times \cdots \times X_{m-1} .
$$

Since $K_{m-1}$ is $m-1$ disjoint vertices, the definition of the polyhedral product implies that

$$
(\underline{C X}, \underline{X})^{K_{m-1}}=\bigcup_{i=1}^{m-1} X_{1} \times \cdots \times X_{i-1} \times C X_{i} \times X_{i+1} \times \cdots \times X_{m-1} .
$$


Further, the definition of the polyhedral product implies that the map $(\underline{C X}, \underline{X})^{\emptyset} \stackrel{j}{\longrightarrow}(\underline{C X}, \underline{X})^{K_{m-1}}$ is the inclusion. In particular, $j$ factors as the composite of inclusions

$$
\begin{aligned}
j: X_{1} \times \cdots \times X_{m-1} & \longrightarrow C X_{1} \times X_{2} \times \cdots \times X_{m-1} \\
& \hookrightarrow \bigcup_{i=1}^{m-1} X_{1} \times \cdots \times X_{i-1} \times C X_{i} \times X_{i+1} \times \cdots \times X_{m-1} .
\end{aligned}
$$

Since $C X_{1}$ is contractible, the first map in the composite for $j$ is null homotopic in the first coordinate, so $j$ is homotopic to the composite

$$
\begin{aligned}
X_{1} \times \cdots \times X_{m-1} & \stackrel{\text { proj }}{\longrightarrow} X_{2} \times \cdots \times X_{m-1} \\
& \hookrightarrow X_{1} \times \cdots \times X_{m-1} \\
& \stackrel{j}{\longrightarrow} \bigcup_{i=1}^{m-1} X_{1} \times \cdots \times X_{i-1} \times C X_{i} \times X_{i+1} \times \cdots \times X_{m-1} .
\end{aligned}
$$

Repeating this argument for each coordinate $2 \leq i \leq m-1$ we obtain a factorization of $j$ through the map $X_{1} \times \cdots \times X_{m-1} \stackrel{\text { proj }}{\longrightarrow} * \hookrightarrow X_{1} \times \cdots \times X_{m}$, implying that $j$ is null homotopic.

Thus by Lemma 6.2 there is a homotopy equivalence

$$
(\underline{C X}, \underline{X})^{K_{m}} \simeq\left((\underline{C X}, \underline{X})^{\emptyset} * X_{m}\right) \vee\left((\underline{C X}, \underline{X})^{K_{m-1}} \rtimes X_{m}\right) .
$$

Since $(\underline{C X}, \underline{X})^{K_{m-1}}$ is a suspension by inductive hypothesis, there is a refined homotopy equivalence

$$
(\underline{C X}, \underline{X})^{K_{m}} \simeq\left((\underline{C X}, \underline{X})^{\emptyset} * X_{m}\right) \vee(\underline{C X}, \underline{X})^{K_{m-1}} \vee\left((\underline{C X}, \underline{X})^{K_{m-1}} \wedge X_{m}\right) .
$$

Substituting in the inductively assumed homotopy equivalence for $(\underline{C X}, \underline{X})^{K_{m-1}}$, using the fact that $(\underline{C X}, \underline{X})^{\emptyset}=X_{1} \times \cdots \times X_{m-1}$, and using Lemma 4.2 to decompose $(\underline{C X}, \underline{X})^{\emptyset} * X_{m} \simeq \Sigma(\underline{C X}, \underline{X})^{\emptyset} \wedge X_{m}$, we obtain the asserted homotopy equivalence for $(\underline{C X}, \underline{X})^{K_{m}}$.

It may be worth noting that in terms of the strategy for identifying the homotopy type of $(\underline{C X}, \underline{X})^{K}$, if $\mathcal{K}$ is the family of simplicial complexes consisting of disjoint vertices, then the argument for the proof of Proposition 7.1 shows that as $K_{m} \in \mathcal{K}$ we have: (i) the map $(\underline{C X}, \underline{X})^{\operatorname{link}_{K}(m) \stackrel{j}{\longrightarrow}}$ $(\underline{C X}, \underline{X})^{K \backslash\{m\}}$ is null homotopic, (ii) $\operatorname{link}_{K_{m}}(m)$ and $K_{m} \backslash\{m\}$ are both in $\mathcal{K}$, and (iii) $(\underline{C X}, \underline{X})^{\operatorname{link}_{K}(m)}$ is a suspension.

Proposition 7.1 generalizes a result of Porter [35]. His work long predates the notion of a polyhedral product but in modern language he proved 
Proposition 7.1 in the case of $(\underline{C \Omega X}, \underline{\Omega X})^{K_{m}}$. It also desuspends the homotopy decomposition of $\Sigma(\underline{C X}, \underline{X})^{K_{m}}$ in Theorem 4.6.

This leads to a natural question. For which simplicial complexes does the homotopy decomposition of $\Sigma(\underline{C X}, \underline{X})^{K}$ in Theorem 4.6 desuspend? The case when $K$ is shifted was done in special cases of $(\underline{C X}, \underline{X})$ in $[18,21]$ and in general in [19,22]. In [23], a desuspension was proved for a strictly larger family of simplicial complexes called sequential Cohen-Macauley. In [3, 23] it was shown that a desuspension exists if and only if $(\underline{C X}, \underline{X})^{K}$ is a co$H$-space, and near characterizations of when this occurs were given. The strategy for desuspending the decomposition in Theorem 4.6 in the shifted case was exactly the strategy that we have been working with: the shifted property implies - after some work - that there is a reordering of the vertices with the property that the map $(\underline{C X}, \underline{X})^{\operatorname{link}_{K}(m)} \stackrel{j}{\longrightarrow}(\underline{C X}, \underline{X})^{K \backslash\{m\}}$ is null homotopic, and it is not difficult to see that the shifted property is inherited by both $\operatorname{link}_{K}(m)$ and $K \backslash\{m\}$. We record the outcome without proving it.

Theorem 7.2: Let $K$ be a shifted simplicial complex on the vertex set $[\mathrm{m}]$ and let $X_{1}, \ldots, X_{m}$ be pointed, path-connected $C W$-complexes. Then the homotopy decomposition in Theorem 4.6 desuspends to give a homotopy equivalence

$$
(\underline{C X}, \underline{X})^{K} \simeq \bigvee_{I \notin K} \Sigma\left(\left|K_{I}\right| \wedge \widehat{X}^{I}\right)
$$

In the special case of the moment-angle complex $\mathcal{Z}_{K}$, we obtain the following corollary.

Corollary 7.3: Let $K$ be a shifted simplicial complex on the vertex set $[\mathrm{m}]$. There is a homotopy equivalence

$$
\mathcal{Z}_{K} \longrightarrow \bigvee_{I \notin K} \Sigma^{|I|+1}\left|K_{I}\right|
$$

In particular, as in [1], if $K$ is shifted and $I \subseteq[m]$ then the geometric realization $\left|K_{I}\right|$ is homotopy equivalent to a wedge of spheres. So when $K$ is shifted $\mathcal{Z}_{K}$ is homotopy equivalent to a wedge of spheres.

Example 7.4: Return to Example 4.8. It was shown that if $K$ is the 
simplicial complex

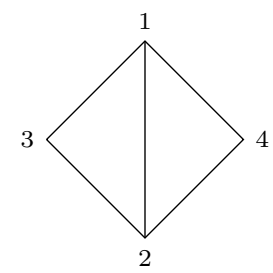

$K$

then there is a homotopy equivalence

$$
\Sigma \mathcal{Z}_{K} \simeq S^{4} \vee S^{6} \vee S^{6} \vee S^{7} \vee S^{7}
$$

By Example 5.3, $K$ is shifted. Let's go through the argument to desuspend the homotopy equivalence for $\Sigma \mathcal{Z}_{K}$. It is clearer to do the more general $(\underline{C X}, \underline{X})$ case as that more naturally keeps track of coordinate. Recall from Example 5.1 that the star, link and restriction of $K$ for the vertex 1 are the simplicial complexes:

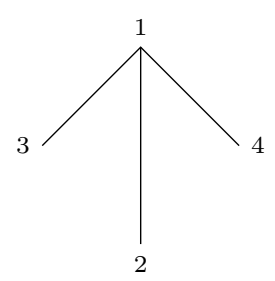

$\operatorname{star}_{K}(1)$

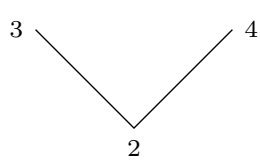

$K \backslash\{1\}$

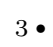

$\dot{2}$

$\operatorname{link}_{K}(1)$

However, as will be more clear by the end of the next section, the fact that the missing face $(3,4)$ of $\operatorname{link}_{K}(1)$ is also a missing face of $K \backslash\{1\}$ implies

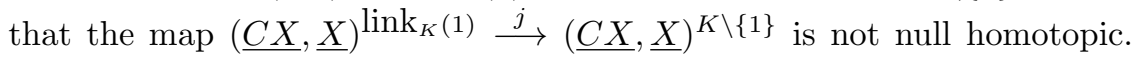
Instead, we consider a different vertex. Note that choosing the vertex 2 as the starting point gives star, link and restriction diagrams similar to those 
for 1 . So instead let's start with vertex 3 . Then we have:

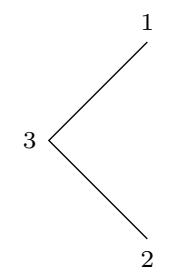

$\operatorname{star}_{K}(3)$

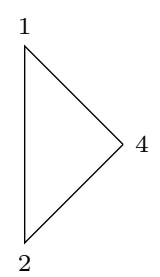

$K \backslash\{3\}$

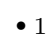

$\dot{2}$

$\operatorname{link}_{K}(3)$

Note here that $\operatorname{link}_{K}(3)$ must be regarded as a simplicial complex on the vertex set $\{1,2,4\}$, the same vertex set as $K \backslash\{3\}$. Let $L$ be $\operatorname{link}_{K}(3)$ regarded as a simplicial complex on the vertex set $\{1,2\}$. Then $(\underline{C X}, \underline{X})^{\operatorname{link}_{K}(3)}=$ $(\underline{C X}, \underline{X})^{L} \times X_{4}$. As $L$ is two disjoint points, by Example $2.2,(\underline{C X}, \underline{X})^{L} \simeq$ $X_{1} * X_{2}$. Therefore

$$
(\underline{C X}, \underline{X})^{\operatorname{link}_{K}(3)}=\left(X_{1} * X_{2}\right) \times X_{4} .
$$

Observe next that the full subcomplex of $K \backslash\{3\}$ on the vertex

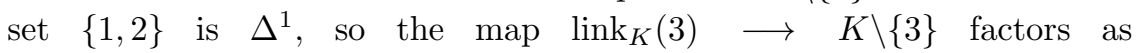
the composite $\operatorname{link}_{K}(3) \longrightarrow \Delta^{1} \longrightarrow K \backslash\{3\}$, implying that the map $(\underline{C X}, \underline{X})^{\operatorname{link}_{K}(3)} \stackrel{j}{\longrightarrow}(\underline{C X}, \underline{X})^{K \backslash\{3\}}$ factors as the composite $(\underline{C X}, \underline{X})^{\operatorname{link}_{K}(3)} \longrightarrow(\underline{C X}, \underline{X})^{\Delta^{1}} \longrightarrow(\underline{C X}, \underline{X})^{K \backslash\{3\}}$. But by the definition of the polyhedral product, $(\underline{C X}, \underline{X})^{\Delta^{1}}=C X_{1} \times C X_{2}$ is contractible. Therefore $j$ is null homotopic. Thus by Lemma 6.2 there is a homotopy equivalence

$$
(\underline{C X}, \underline{X})^{K} \simeq\left((\underline{C X}, \underline{X})^{\operatorname{link}_{K}(3)} * X_{3}\right) \vee\left((\underline{C X}, \underline{X})^{K \backslash\{3\}} \rtimes X_{3}\right) .
$$

We have already mentioned that $(\underline{C X}, \underline{X})^{\operatorname{link}_{K}(3)} \simeq\left(X_{1} * X_{2}\right) \times X_{4}$. Thus

$$
(\underline{C X}, \underline{X})^{\operatorname{link}_{K}(3)} * X_{3} \simeq\left[\left(X_{1} * X_{2}\right) \times X_{4}\right] * X_{3} .
$$

Using the fact that $A * B \simeq \Sigma A \wedge B$ and Lemma 4.1 to decompose the suspension of a product, we obtain homotopy equivalences

$$
\begin{aligned}
(\underline{C X}, \underline{X})^{\operatorname{link}_{K}(3)} * X_{3} & \simeq \Sigma\left[\left(\Sigma X_{1} \wedge X_{2}\right) \times X_{4}\right] \wedge X_{3} \\
& \simeq\left[\left(\Sigma^{2} X_{1} \wedge X_{4}\right) \vee \Sigma X_{4} \vee\left(\Sigma^{2} X_{1} \wedge X_{2} \wedge X_{4}\right)\right] \wedge X_{3} \\
& \simeq\left(\Sigma^{2} X_{1} \wedge X_{2} \wedge X_{3}\right) \vee\left(\Sigma X_{3} \wedge X_{4}\right) \\
& \vee\left(\Sigma^{2} X_{1} \wedge X_{2} \wedge X_{3} \wedge X_{4}\right) .
\end{aligned}
$$


On the other hand, as we will see in the next section, $(\underline{C X}, \underline{X})^{K \backslash\{3\}} \simeq$ $X_{1} * X_{2} * X_{4} \simeq \Sigma^{2} X_{1} \wedge X_{2} \wedge X_{4}$. Thus there is a homotopy equivalence

$$
(\underline{C X}, \underline{X})^{K \backslash\{3\}} \rtimes X_{3} \simeq\left(\Sigma^{2} X_{1} \wedge X_{2} \wedge X_{4}\right) \vee\left(\Sigma^{2} X_{1} \wedge X_{2} \wedge X_{3} \wedge X_{4}\right) .
$$

Hence there is a homotopy equivalence

$$
\begin{aligned}
(\underline{C X}, \underline{X})^{K} \simeq\left(\Sigma^{2} X_{1} \wedge\right. & \left.X_{2} \wedge X_{4}\right) \vee\left(\Sigma X_{3} \wedge X_{4}\right) \vee\left(\Sigma^{2} X_{1} \wedge X_{2} \wedge X_{3} \wedge X_{4}\right) \\
& \vee\left(\Sigma^{2} X_{1} \wedge X_{2} \wedge X_{3}\right) \vee\left(\Sigma^{2} X_{1} \wedge X_{2} \wedge X_{3} \wedge X_{4}\right) .
\end{aligned}
$$

In particular, in the case of $\mathcal{Z}_{K}$ each $X_{i}=S^{1}$, so we obtain a homotopy equivalence

$$
\mathcal{Z}_{K} \simeq S^{5} \vee S^{3} \vee S^{6} \vee S^{5} \vee S^{6}
$$

as desired.

\section{Higher Whitehead products and minimal missing faces}

Consider the homotopy fibration

$$
(\underline{C \Omega X}, \underline{\Omega X})^{K} \longrightarrow(\underline{X}, \underline{*})^{K} \longrightarrow \prod_{i=1}^{m} X_{i}
$$

from Corollary 3.14. At this point the homotopy type of $(\underline{C \Omega X}, \underline{\Omega X})^{K}$ has been identified for various simplicial complexes $K$. However, nothing has been said about the homotopy type of the map $(\underline{C \Omega X}, \underline{\Omega X})^{K} \longrightarrow$ $(\underline{X}, \underline{*})^{K}$. This is the problem addressed in this section. In $[20,16,24]$ it has been determined that Whitehead products and higher Whitehead products play an important role. In particular, the existence of nontrivial higher Whitehead products in $(\underline{X}, \underline{*})^{K}$ corresponds to the existence of minimal missing faces in $K$. We begin with a discussion of the standard Whitehead product.

\subsection{Whitehead products}

One way of defining a Whitehead product is as the adjoint of a Samelson product. Let $Y$ be an $H$-group, that is, $Y$ is a homotopy associative $\mathrm{H}$ space with a homotopy inverse. Let $\bar{c}: Y \times Y \longrightarrow Y$ be the commutator of $Y$, defined pointwise by $\bar{c}(x, y)=x y x^{-1} y^{-1}$. Observe that the restriction of $\bar{c}$ to the wedge is null homotopic, so $\bar{c}$ extends to a map

$$
c: Y \wedge Y \longrightarrow Y \text {. }
$$


By Lemma 4.1, the quotient map $Y \times Y \longrightarrow Y \wedge Y$ has a right homotopy inverse after suspending. Therefore the homotopy class of $c$ is determined uniquely by the homotopy class of $\bar{c}$. Let $a: A \longrightarrow Y$ and $b: B \longrightarrow Y$ be maps. The composite

$$
\langle a, b\rangle: A \wedge B \stackrel{a \wedge b}{\longrightarrow} Y \wedge Y \stackrel{c}{\longrightarrow} Y
$$

is the Samelson product of $a$ and $b$. In particular, $c$ itself is the Samelson product of the identity map on $Y$ with itself. The Samelson product has many useful properties, including the fact that if $x \in H_{*}(A)$ and $y \in H_{*}(B)$ are primitive then $(\langle a, b\rangle)_{*}(x, y)=[a(x), b(y)]$, where $[\cdot, \cdot]$ is the graded commutator in the homology algebra $H_{*}(Y)$.

Now suppose that $X$ is a space and there are maps $f: \Sigma A \longrightarrow X$ and $g: \Sigma B \longrightarrow X$. Let $\tilde{f}: A \longrightarrow \Omega X$ and $\tilde{g}: B \longrightarrow \Omega X$ be the adjoints of $f$ and $g$ respectively. We may take the Samelson product $\langle\tilde{f}, \tilde{g}\rangle: A \wedge B \longrightarrow$ $\Omega X$. Its adjoint is a map

$$
[f, g]: \Sigma A \wedge B \longrightarrow X
$$

called the Whitehead product of $f$ and $g$.

Whitehead products play a crucial role in homotopy theory. One key context in which they arise is the following. Let $X$ and $Y$ be pointed, simply-connected spaces and consider the inclusion $X \vee Y \longrightarrow X \times Y$. By Proposition 7.1, the homotopy fibre of this inclusion is homotopy equivalent to $\Sigma \Omega X \wedge \Omega Y$. Ganea [14] proved a much sharper result by identifying the homotopy class of the map from the fibre to $X \vee Y$. Let $i_{L}: X \longrightarrow X \vee Y$ and $i_{R}: Y \longrightarrow X \vee Y$ be the inclusions of the left and right wedge summands respectively. Let $e v_{L}$ and $e v_{R}$ be the composites $e v_{L}: \Sigma \Omega X \stackrel{e v}{\longrightarrow} X \stackrel{i_{L}}{\longrightarrow}$ $X \vee Y$ and $e v_{R}: \Sigma \Omega Y \stackrel{e v}{\longrightarrow} Y \stackrel{i_{R}}{\longrightarrow} X \vee Y$ respectively, where $e v$ is the canonical evaluation map.

Theorem 8.1: (Ganea) Let $X$ and $Y$ be pointed, simply-connected spaces. Then there is a homotopy fibration

$$
\Sigma \Omega X \wedge \Omega Y \stackrel{\left[e v_{L}, e v_{R}\right]}{\longrightarrow} X \vee Y \longrightarrow X \times Y .
$$

One consequence is the following. In general, let $E: X \longrightarrow \Omega \Sigma X$ be the suspension map, which is adjoint to the identity map on $\Sigma X$. If $X=\Sigma A$ and $Y=\Sigma B$ then the composite $\Sigma A \wedge B \stackrel{\Sigma E \wedge E}{\longrightarrow} \Sigma \Omega \Sigma A \wedge \Omega \Sigma B \stackrel{\left[e v_{L}, e v_{R}\right]}{\longrightarrow}$ $\Sigma A \vee \Sigma B$ is homotopic to the Whitehead product $\left[i_{L}, i_{R}\right]$, and the homotopy cofibre of this map is $\Sigma A \times \Sigma B$. 


\subsection{Higher Whitehead products}

Attaching by a Whitehead product is what is needed to produce $\Sigma A \times \Sigma B$ from $\Sigma A \vee \Sigma B$. Theorem 8.1 says that there is a generalization when looking at the homotopy fibre of any wedge into a product. To get higher versions of Whitehead products, we should look at what is needed to produce $\prod_{i=1}^{m} \Sigma A_{i}$ from the fat wedge on $\Sigma A_{1}, \ldots, \Sigma A_{m}$, and then generalize by looking at the homotopy fibre of any fat wedge into a product. To make this precise some definitions are needed.

Definition 8.2: For spaces $X_{1}, \ldots, X_{m}$, let $F W(1, \ldots, m)$ be the subspace of $X_{1} \times \cdots \times X_{m}$ defined by

$$
F W(1, \ldots, m)=\bigcup_{i=1}^{m} X_{1} \times \cdots \times X_{i-1} \times * \times X_{i+1} \times \cdots \times X_{m} .
$$

The space $F W(1, \ldots, m)$ is the fat wedge of $X_{1}, \ldots, X_{m}$.

By analogy with Theorem 8.1, a higher Whitehead product should be defined via the homotopy fibre of the inclusion of $F W(1, \ldots, m)$ into $X_{1} \times \cdots \times X_{m}$. To do so, it is easier to phrase this homotopy fibration in terms of polyhedral products. Let $\partial \Delta^{m-1}$ be the boundary of $\Delta^{m-1}$. Note that $\partial \Delta^{m-1}$ is the full $m-2$-skeleton of $\Delta^{m-1}$. Therefore, comparing the definition of the polyhedral product for $(\underline{X}, \underline{*})^{\Delta^{m-1}}$ and that for $F W(1, \ldots, m)$ we obtain

$$
F W(1, \ldots, m)=(\underline{X}, \underline{*})^{\partial \Delta^{m-1}} .
$$

So by Corollary 3.14 there is a homotopy fibration

$$
(\underline{C \Omega X}, \underline{\Omega X})^{\partial \Delta^{m-1}} \longrightarrow F W(1, \ldots, m) \longrightarrow \prod_{i=1}^{m} X_{i} .
$$

Thus we want to identity the homotopy type of $(\underline{C \Omega X}, \underline{\Omega X})^{\partial \Delta^{m-1}}$. We will do this in the more general case of $(\underline{C X}, \underline{X})^{\partial \Delta^{m-1}}$.

Lemma 8.3: For $k \geq 2$, there is a pushout of simplicial complexes

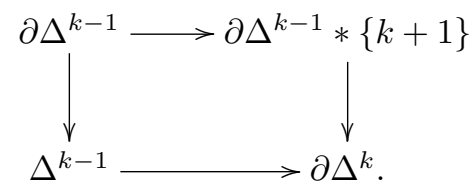


Proof: If $K$ is a simplicial complex on the index set $[k]$, regard the faces of $K$ as subsequences $\left(i_{1}, \ldots, i_{\ell}\right)$ where $1 \leq i_{1}<\cdots<i_{\ell} \leq k$. Observe that $\Delta^{k}$ has as faces all subseqences of $\{1, \ldots, k+1\}$, and $\partial \Delta^{k}$ has as faces all proper subsequences of $\{1, \ldots, k+1\}$. In particular, $\partial \Delta^{k}$ is the union of: (i) all subsequences of the form $\left(i_{1}, \ldots, i_{\ell}\right)$ where $1 \leq i_{1}<\cdots<i_{\ell} \leq k$ - that is, those subsequences corresponding to $\Delta^{k-1}$, and (ii) all the subsequences of the form $\left(i_{1}, \ldots, i_{\ell}, k+1\right)$ where $1 \leq i_{1}<\cdots<i_{\ell} \leq k$ but at least one $i_{j}$ is missing - that is, those subsequences corresponding to $\partial \Delta^{k-1} *\{k+1\}$. The intersection of the subsequences in (i) and (ii) consists of all subsequences of the form $\left(i_{1}, \ldots, i_{\ell}\right)$ where $1 \leq i_{1}<\cdots<i_{\ell} \leq k$ and at least one $i_{j}$ is missing - that is, those subsequences corresponding to $\partial \Delta^{k-1}$. This proves the lemma.

Remark 8.4: The proof of Lemma 8.3 shows that the pushout in the statement of the lemma is the usual star-link-restriction pushout in the case of $K=\partial \Delta^{k}$. That is, $\operatorname{star}_{\partial \Delta^{k}}=\partial \Delta^{k-1} *\{k+1\}, \operatorname{link}_{\partial \Delta^{k}}=\partial \Delta^{k-1}$ and $\left(\partial \Delta^{k}\right) \backslash\{k+1\}=\Delta^{k-1}$.

Remark 8.4 implies that, by Proposition 5.6, there is a corresponding pushout of polyhedral products

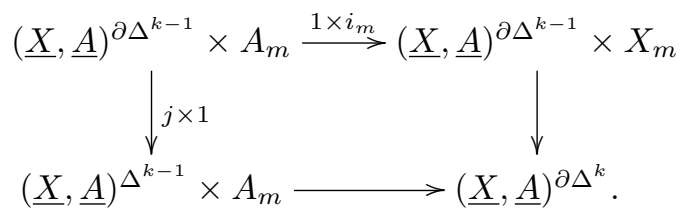

Consider the special case of $(\underline{X}, \underline{*})$. Then $(\underline{X}, \underline{*})^{\Delta^{k-1}}=X_{1} \times \cdots \times X_{k}$, $(\underline{X}, \underline{*})^{\partial \Delta^{k-1}}=F W(1, \ldots, k)$, and $(\underline{X}, \underline{*})^{\partial \Delta^{k}}=F W(1, \ldots, k+1)$. Phrased in terms of a larger vertex set, diagram (8.2) implies the following.

Lemma 8.5: Let $\left\{i_{1}, \ldots, i_{k}\right\}$ be a subset of $\{1, \ldots, m-1\}$ with the property that $1 \leq i_{1}<\cdots<i_{k} \leq m-1$. Then there is a pushout

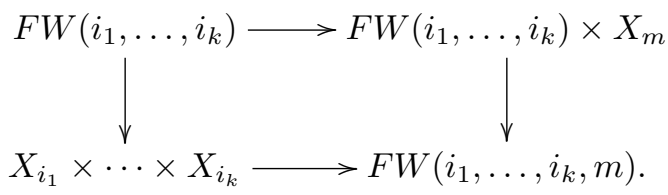


Remark 8.6: Lemma 8.5 has a direct proof as well. By definition of the fat wedge,

$$
\begin{gathered}
F W\left(i_{1}, \ldots, i_{k}, m\right)=\left(\bigcup_{j=1}^{k} X_{i_{1}} \times \cdots \times X_{i_{j-1}} \times * \times X_{i_{j+1}} \times \cdots \times \cdots X_{i_{k}} \times X_{m}\right) \\
\cup\left(X_{i_{1}} \times \cdots \times X_{i_{k}} \times *\right) .
\end{gathered}
$$

Notice that the first term in this union is $F W\left(i_{1}, \ldots, i_{k}\right) \times X_{m}$, the second term is $X_{i_{1}} \times \cdots \times X_{i_{k}}$, and the union is taken over the common subspace $F W\left(i_{1}, \ldots, i_{k}\right)$. The asserted pushout now follows immediately.

Next, consider the special case of $(\underline{C X}, \underline{X})$. By (8.2) and Corollary 5.7, for $m \geq 2$ there is a pushout of polyhedral products

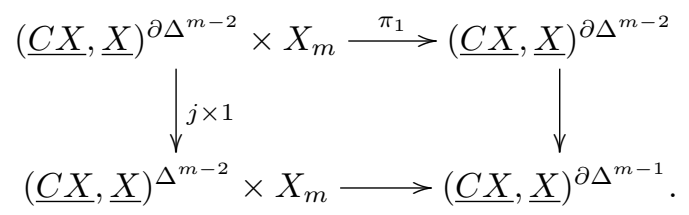

Lemma 8.7: For $m \geq 2$ there is a homotopy equivalence

$$
(\underline{C X}, \underline{X})^{\partial \Delta^{m-1}} \simeq \Sigma^{m-1} X_{1} \wedge \cdots \wedge X_{m} .
$$

Proof: The proof is by induction on the number of vertices. When $m=2$ then $\partial \Delta^{1}$ is two disjoint vertices, so by Example 2.2 we have $(\underline{C X}, \underline{X})^{\partial \Delta^{1}} \simeq$ $\Sigma X_{1} \wedge X_{2}$. Assume inductively that $(\underline{C X}, \underline{X})^{\partial \Delta^{m-2}} \simeq \Sigma^{m-2} X_{1} \wedge \cdots \wedge X_{m-1}$. By Example 2.3, $(\underline{C X}, \underline{X})^{\Delta^{m-1}}=C X_{1} \times \cdots \times C X_{m-1} \simeq *$, so the homotopy pushout (8.3) is equivalent to the homotopy pushout

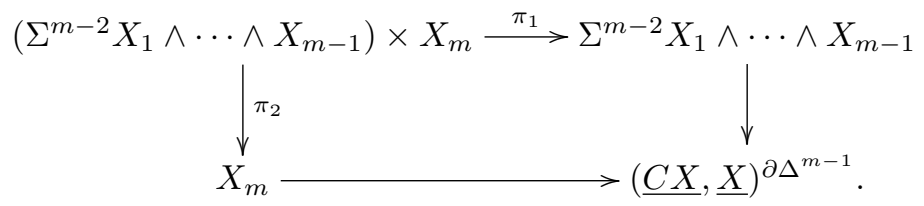

Therefore, by Lemma 6.1, there are homotopy equivalences $(\underline{C X}, \underline{X})^{\partial \Delta^{m-1}} \simeq\left(\Sigma^{m-2} X_{1} \wedge \cdots \wedge X_{m-1}\right) * X_{m} \simeq \Sigma^{m-1} X_{1} \wedge \cdots \wedge X_{m}$

By Lemma 8.7, the homotopy fibration (8.1) can be rewritten as a homotopy fibration

$$
\Omega X_{1} * \cdots * \Omega X_{m} \stackrel{\phi_{m}}{\longrightarrow} F W(1, \ldots, m) \longrightarrow X_{1} \times \cdots \times X_{m}
$$


for some map $\phi_{m}$. This lets us define higher Whitehead products.

Definition 8.8: For $1 \leq i \leq m$, suppose that there are maps $f_{i}: X_{i} \longrightarrow Y$ for some space $Y$. Suppose also that there is an extension of the wedge $\bigvee_{i=1}^{m} X_{i} \stackrel{\bigvee_{i=1}^{m} f_{i}}{\longrightarrow} Y$ to a map $\bar{f}: F W(1, \ldots, m) \longrightarrow Y$. Then the composite

$$
\Omega X_{1} * \cdots * \Omega X_{m} \stackrel{\phi_{m}}{\longrightarrow} F W(1, \ldots, m) \stackrel{\bar{f}}{\longrightarrow} Y
$$

is a higher Whitehead product.

Observe that if $m=2$ then $\bar{f}=f_{1} \vee f_{2}$ and $\phi_{2}$ is the ordinary Whitehead product $\left[e v_{L}, e v_{R}\right]$. So the homotopy class of $\bar{f} \circ \phi_{2}$ is determined by the homotopy classes of $f_{1}$ and $f_{2}$. However, if $m \geq 3$ then there may be different choices of an extension $\bar{f}$ of $\bigvee_{i=1}^{m} f_{i}$, so the homotopy class of the higher Whitehead product $\bar{f} \circ \phi_{m}$ need not be determined only by the homotopy classes of $f_{1}, \ldots, f_{m}$.

\subsection{Relating higher Whitehead products to minimal missing faces}

Let $K$ be a simplicial complex on the vertex set $[m]$. We use the homotopy fibration

$$
(\underline{C \Omega X}, \underline{\Omega X})^{K} \longrightarrow(\underline{X}, \underset{*}{*})^{K} \longrightarrow \prod_{i=1}^{m} X_{i}
$$

from Corollary 3.14 to establish a correspondence between the minimal missing faces in $K$ and nontrivial higher Whitehead products on $(\underline{X}, \underline{*})^{K}$. This requires some definitions.

Definition 8.9: Let $K$ be a simplicial complex on the vertex set $[m]$. A subset $\sigma=\left(i_{1}, \ldots, i_{k}\right)$ of $[m]$ is a missing face of $K$ if $\sigma \notin K$. It is a minimal missing face if any proper subset of $\sigma$ is a face of $K$. Equivalently, $\sigma$ is a minimal missing face of $K$ if $\partial \sigma \subseteq K$ but $\sigma \notin K$. 
Example 8.10: Let $K$ be the following simplical complex:

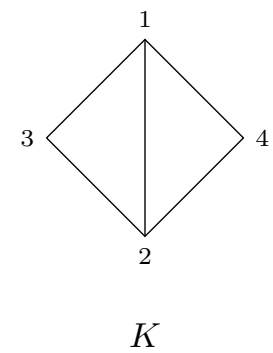

The missing faces of $K$ are

$$
M F(K)=\{(3,4),(1,2,3),(1,2,4),(1,3,4),(2,3,4),(1,2,3,4)\} .
$$

As $(3,4)$ is a proper face of $(1,3,4),(2,3,4)$ and $(1,2,3,4)$ which is missing from $K$, none of $(1,3,4),(2,3,4)$ or $(1,2,3,4)$ are minimal missing faces. The complete list of minimal missing faces of $K$ is

$$
M M F(K)=\{(3,4),(1,2,3),(1,2,4)\} .
$$

Let $K$ be a simplicial complex on the vertex set $[m]$. Recall that $K_{m}$ is the simplicial complex consisting of $m$ disjoint points. Including the vertices into $K$ gives a simplicial map $K_{m} \longrightarrow K$. This induces a map of polyhedral products $(\underline{X}, \underline{*})^{K_{m}} \longrightarrow(\underline{X}, \underline{*})^{K}$. By Example $2.1,(\underline{X}, \underline{*})^{K_{m}}=\bigvee_{i=1}^{m} X_{i}$, so the inclusion of $K_{m}$ into $K$ induces a map

$$
F_{m}: \bigvee_{i=1}^{m} X_{i} \longrightarrow(\underline{X}, \underline{*})^{K} .
$$

Lemma 8.11: Let $K$ be a simplicial complex on the vertex set $[m]$. Then $\sigma=\left(i_{1}, \ldots, i_{k}\right)$ is a minimal missing face of $K$ if and only if the composite $\bigvee_{j=1}^{k} X_{i_{j}} \hookrightarrow \bigvee_{i=1}^{m} X_{i} \stackrel{F_{m}}{\longrightarrow}(\underline{X}, \underline{*})^{K}$ extends to a map $F W\left(i_{1}, \ldots, i_{k}\right) \longrightarrow$ $(\underline{X}, \underline{*})^{K}$ but does not extend to a map $\prod_{j=1}^{k} X_{j_{k}} \longrightarrow(\underline{X}, \underline{*})^{K}$.

Proof: By definition, $\sigma=\left(i_{1}, \ldots, i_{k}\right)$ is a minimal missing face of $K$ if and only if $\partial \sigma \subseteq K$ but $\sigma \notin K$. That is, if and only if there is a map of simplicial complexes $\partial \sigma \longrightarrow K$ which does not extend to a map $\sigma \longrightarrow$ $K$. By definition of the polyhedral product, $(\underline{X}, \underline{*})^{K}$ is the union of the coordinate subspaces formed from the faces of $K$. That is, $X_{j_{1}} \times \cdots \times X_{j_{t}}$ is a coordinate subspace of $(\underline{X}, \underline{*})^{K}$ if and only if the face $\tau=\left(j_{1}, \ldots, j_{t}\right) \in K$. In our case, since $\sigma$ is a $(k-1)$-simplex, the simplicial map $\partial \sigma \longrightarrow K$ exists if and only if every coordinate subspace of the fat wedge $F W\left(i_{1}, \ldots, i_{k}\right)$ is 
in $(\underline{X}, \underline{*})^{K}$. That is, if and only if there is a map $F W\left(i_{1}, \ldots, i_{k}\right) \longrightarrow(\underline{X}, \underline{*})^{K}$ extending the inclusion of the coordinates $\bigvee_{j=1}^{k} X_{i_{j}} \longrightarrow(\underline{X}, \underline{*})^{K}$. Further, the simplicial map $\partial \sigma \longrightarrow K$ does not extend to a simplicial map $\sigma \longrightarrow K$ if and only if $X_{1_{1}} \times \cdots \times X_{i_{k}}$ is not a coordinate subspace of $(\underline{X}, \underline{*})^{K}$. That is, if and only if the map $F W\left(i_{1}, \ldots, i_{k}\right) \longrightarrow(\underline{X}, \underline{*})^{K}$ does not extend to a $\operatorname{map} X_{i_{1}} \times \cdots \times X_{i_{k}} \longrightarrow(\underline{X}, \underline{*})^{K}$.

Let $\sigma=\left(i_{1}, \ldots, i_{k}\right)$ be a minimal missing face of $K$. By Lemma 8.11, there is an extension of the composite $\bigvee_{j=1}^{k} X_{i_{j}} \hookrightarrow \bigvee_{i=1}^{m} X_{i} \stackrel{F_{m}}{\longrightarrow}(\underline{X}, \underline{*})^{K}$ to a map $f: F W\left(i_{1}, \ldots, i_{k}\right) \longrightarrow(\underline{X}, \underline{*})^{K}$. Define the higher Whitehead product $w(\sigma)$ by the composite

$$
w(\sigma): \Omega X_{i_{1}} * \cdots * \Omega X_{i_{k}} \stackrel{\phi_{k}}{\longrightarrow} F W\left(i_{1}, \ldots, i_{k}\right) \stackrel{f}{\longrightarrow}(\underline{X}, \underline{*})^{K} .
$$

In the next Proposition the equivalence in Lemma 8.11 is rephrased in terms of the existence of nontrivial higher Whitehead products.

Proposition 8.12: Let $K$ be a simplicial complex on the vertex set $[\mathrm{m}]$ and let $(\underline{X}, \underline{*})$ be a sequence of pointed $C W$-pairs. Then $\sigma=\left(i_{1}, \ldots, i_{k}\right)$ is a minimal missing face of $K$ if and only if the higher Whitehead product $w(\sigma): \Omega X_{i_{1}} * \cdots * \Omega X_{i_{k}} \stackrel{\phi_{k}}{\longrightarrow} F W\left(i_{1}, \ldots, i_{k}\right) \stackrel{f}{\longrightarrow}(\underline{X}, *)^{K}$ is nontrivial.

Proof: Suppose that $w(\sigma)=f \circ \phi_{k}$ is nontrivial. Since $\phi_{k}$ is the homotopy fibre of the inclusion $F W\left(i_{1}, \ldots, i_{k}\right) \longrightarrow X_{i_{1}} \times \cdots \times X_{i_{k}}$, the nontriviality of $f \circ \phi_{k}$ implies that there can be no extension of $F W\left(i_{1}, \ldots, i_{k}\right) \stackrel{f}{\longrightarrow}(\underline{X}, \underline{*})^{K}$ to a map $g: X_{i_{1}} \times \cdots \times X_{i_{k}} \longrightarrow(\underline{X}, \underline{*})^{K}$. For otherwise $f \circ \phi_{k}$ would factor through two consecutive maps in a homotopy fibration and so be null homotopic. Therefore, as $f$ does not extend to $g$, by Lemma $8.11 \sigma$ is a minimal missing face of $K$.

Conversely, suppose that $w(\sigma)$ is null homotopic. We wish to show that $\sigma$ is not a minimal missing face of $K$. This case is trickier for the following reason. If each $X_{i_{j}}$ is a suspension, $X_{i_{j}}=\Sigma X_{i_{j}}^{\prime}$, then the composite

$$
X_{i_{1}}^{\prime} * \cdots * X_{i_{k}}^{\prime} \stackrel{\bar{E}}{\longrightarrow} \Omega \Sigma X_{i_{1}}^{\prime} * \cdots * \Omega \Sigma X_{i_{k}}^{\prime} \stackrel{\phi_{k}}{\longrightarrow} F W\left(i_{1}, \ldots, i_{k}\right)
$$

is the attaching map for the product $\Sigma X_{i_{1}}^{\prime} \times \cdots \times \Sigma X_{i_{k}}^{\prime}$, where $\bar{E}=E * \cdots * E$. Therefore, $f$ extends to $g$ in this case if and only if $f \circ \phi_{k} \circ \bar{E}$ is null homotopic. In particular, if $w(\sigma)=f \circ \phi_{k}$ is null homotopic then $f \circ \phi_{k} \circ \bar{E}$ is null homotopic, implying that $f$ extends to $g$, and so by Lemma 8.11, $\sigma$ is not a minimal missing face of $K$. However, in the general case $X_{i_{j}}$ may 
not be a suspension, so the product $X_{i_{1}} \times \cdots \times X_{i_{k}}$ may not be the cofibre of a map into the $F W\left(i_{1}, \ldots, i_{m}\right)$.

Instead, for each $1 \leq j \leq k$, let $g_{j}: S^{n_{j}} \longrightarrow X_{i_{j}}$ be the inclusion of a bottom cell. Let $F W(\underline{S})$ be the fat wedge on $S^{n_{1}} \times \cdots \times S^{n_{k}}$. By Proposition 3.1 (a), the polyhedral product is natural with respect to the maps $g_{j}$, so we obtain a homotopy fibration diagram

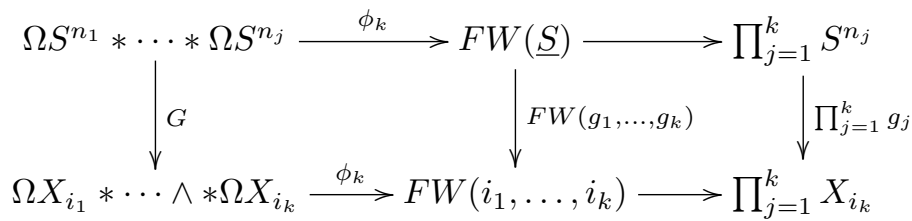

where $G=\Omega g_{1} * \cdots * \Omega g_{k}$.

Now suppose that $w(\sigma)=f \circ \phi_{k}$ is null homotopic. Then the composite $f \circ \phi_{k} \circ G$ is null homotopic, implying by the homotopy commutativity of the left square in (8.4) that $f \circ F W\left(g_{1}, \ldots, g_{k}\right) \circ \phi_{k}$ is null homotopic. Notice that this is also a higher Whitehead product $\bar{f} \circ \phi_{k}$, where $\bar{f}=f \circ F W\left(g_{1}, \ldots, g_{k}\right)$. Since each $S^{n_{j}}$ is a suspension, by the argument above, the null homotopy for $\bar{f} \circ \phi_{k}$ implies that $\sigma$ is not a minimal missing face of $K$, and we are done.

Example 8.13: Return to Example 8.10. By Proposition 8.12, the minimal missing face $(3,4)$ corresponds to a nontrivial Whitehead product $\Omega X_{3} * \Omega X_{4} \longrightarrow(\underline{X}, \underline{*})^{K}$. The minimal missing faces $(1,2,3)$ and $(1,2,4)$ correspond to nontrivial higher Whitehead products $\Omega X_{1} * \Omega X_{2} * \Omega X_{3} \stackrel{\phi_{3}}{\longrightarrow}$ $F W(1,2,3) \longrightarrow(\underline{X}, *)^{K}$ and $\Omega X_{1} * \Omega X_{2} * \Omega X_{4} \stackrel{\phi_{3}}{\longrightarrow} F W(1,2,4) \longrightarrow$ $(\underline{X}, \underline{*})^{K}$.

To close this section we return to the homotopy fibration $(\underline{C \Omega X}, \underline{\Omega X})^{K} \stackrel{\varphi}{\longrightarrow}(\underline{X}, \underline{*})^{K} \longrightarrow \prod_{i=1}^{m} X_{i}$ and state some results. In [20] it is shown that if $K$ is an " $M F$-complex" and each $X_{i}$ is a suspension, then $(\underline{C \Omega X}, \underline{\Omega X})^{K}$ is homotopy equivalent to a wedge of iterated suspensions of the spaces $\Omega X_{i}$ and the map $\varphi$ is a wedge sum of: higher Whitehead products, one for each minimal missing face of $K$; and iterated Whitehead products formed from one higher Whitehead product and possibly multiple copies of the coordiate inclusions $X_{i} \longrightarrow(\underline{X}, \underline{*})^{K}$. A similar statement is proved for the moment-angle complex $\mathcal{Z}_{K}$ even though $X_{i}=\mathbb{C} P^{\infty}$ is not a suspension. In [24] many of these results are generalized to the case when $K$ is shifted. 


\section{A decomposition of the based loops on a polyhedral product}

In this section we turn to investigating the homotopy type of $\Omega(\underline{X}, \underline{A})^{K}$. We prove a new integral homotopy decomposition for $\Omega(\underline{X}, \underline{A})^{K}$ that holds for any simplicial complex $K$. A different, much finer homotopy decomposition of $\Omega(\underline{X}, \underline{A})^{K}$ can be found in [40]. The advantage of the cruder homotopy decomposition proved here is that the factors are easy to identify in terms of other spaces.

Let $K$ be a simplicial complex on the vertex set $[m]$. Recall from (5.1) that there is a pushout of simplicial complexes

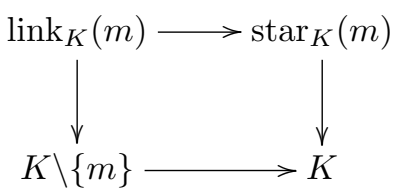

where $\operatorname{star}_{K}(m)=\operatorname{link}_{K}(m) *\{m\}$. Define a new simplicial complex $\bar{K}$ on the vertex set $[m]$ by

$$
\bar{K}=K \backslash\{m\} *\{m\} .
$$

Observe that there is an inclusion of simplicial complexes $K \backslash\{m\} \longrightarrow \bar{K}$ given by including the join factor, so as $\operatorname{star}_{K}(m)=\operatorname{link}_{K}(m) *\{m\}$, there is a pushout map

$$
K \longrightarrow \bar{K} \text {. }
$$

Observe also that $K \backslash\{m\}$ is the full subcomplex of $\bar{K}$ on the vertex set $[m-1]$. That is, $\bar{K} \backslash\{m\}=K \backslash\{m\}$.

Now turn to polyhedral products. By Proposition 5.6, the pushout of simplicial complexes in (9.1) induces a pushout of polyhedral products

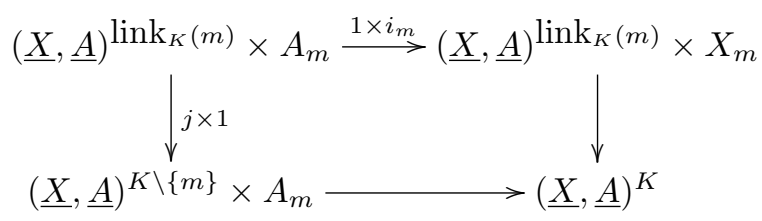

where $i_{m}$ is the inclusion. To relate this to $(\underline{X}, \underline{A})^{\bar{K}}$, observe that the definition of the join of two simplicial complexes implies that if $K=K_{1} * K_{2}$ then there is a homeomorphism

$$
(\underline{X}, \underline{A})^{K} \simeq(\underline{X}, \underline{A})^{K_{1}} \times(\underline{X}, \underline{A})^{K_{2}} .
$$


In particular, as $\bar{K}=K \backslash\{m\} *\{m\}$ there is a homeomorphism

$$
(\underline{X}, \underline{A})^{\bar{K}} \simeq(\underline{X}, \underline{A})^{K \backslash\{m\}} \times X_{m} .
$$

We follow Félix and Tanré's idea [13] of mapping all four corners of the pushout $(9.2)$ into $(\underline{X}, \underline{A})^{\bar{K}}$ and taking homotopy fibres. Mather's Cube Lemma [30] will help identify the homotopy types of the fibres.

Lemma 9.1: (Mather) Suppose that there is a diagram of spaces and maps

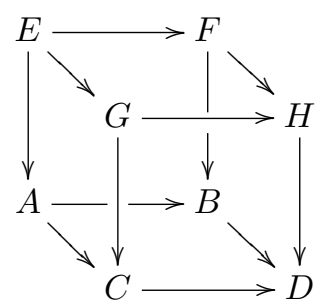

where the bottom face is a homotopy pushout and the four sides are obtained by pulling back with $H \longrightarrow D$. Then the top face is a homotopy pushout.

Consider the map $(\underline{X}, \underline{A})^{K} \longrightarrow(\underline{X}, \underline{A})^{\bar{K}} \simeq(\underline{X}, \underline{A})^{K \backslash\{m\}} \times X_{m}$. Composing from all four corners of the pushout (9.2), we obtain homotopy fibrations

$$
\begin{aligned}
& G_{m} \times Y_{m} \longrightarrow(\underline{X}, \underline{A})^{\operatorname{link}_{K}(m)} \times A_{m} \stackrel{j \times i_{m}}{\longrightarrow}(\underline{X}, \underline{A})^{K \backslash\{m\}} \times X_{m} \\
& Y_{m} \longrightarrow(\underline{X}, \underline{A})^{K \backslash\{m\}} \times A_{m} \stackrel{1 \times i_{m}}{\longrightarrow}(\underline{X}, \underline{A})^{K \backslash\{m\}} \times X_{m} \\
& G_{m} \longrightarrow(\underline{X}, \underline{A})^{\operatorname{link}_{K}(m)} \times X_{m} \stackrel{j \times 1}{\longrightarrow}(\underline{X}, \underline{A})^{K \backslash\{m\}} \times X_{m} \\
& F_{m} \longrightarrow(\underline{X}, \underline{A})^{K} \longrightarrow(\underline{X}, \underline{A})^{K \backslash\{m\}} \times X_{m}
\end{aligned}
$$

where $G_{m}$ is the homotopy fibre of $(\underline{X}, \underline{A})^{\operatorname{link}_{K}(m)} \stackrel{j}{\longrightarrow}(\underline{X}, \underline{A})^{K \backslash\{m\}}, Y_{m}$ is the homotopy fibre of the inclusion $A_{m} \stackrel{i_{m}}{\longrightarrow} X_{m}$, and $F_{m}$ is defined as the homotopy fibre of the map $(\underline{X}, \underline{A})^{K} \longrightarrow(\underline{X}, \underline{A})^{K \backslash\{m\}} \times X_{m}$. By Lemma 9.1, we obtain a homotopy pushout

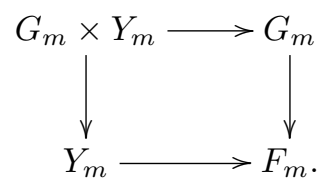


We claim that the maps $G_{m} \times Y_{m} \longrightarrow G_{m}$ and $G_{m} \times Y_{m} \longrightarrow Y_{m}$ in (9.3) are projections. In the first case, the map is induced from Lemma 9.1 by the homotopy pullback diagram

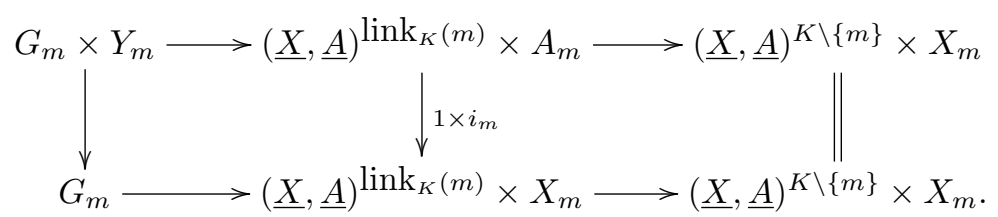

The maps in the right square are defined coordinate-wise, so the homotopy pullback is homotopy equivalent to the topological pullback obtained by turning both $(\underline{X}, \underline{A})^{\operatorname{link}_{K}(m)} \longrightarrow(\underline{X}, \underline{A})^{K \backslash\{m\}}$ and $A_{m} \longrightarrow X_{m}$ into (strict) fibrations. By doing so the map of fibres is also defined coordinatewise, implying that $G_{m} \times Y_{m} \longrightarrow G_{m}$ is homotopic to the projection. Similarly, the map $G_{m} \times Y_{m} \longrightarrow Y_{m}$ is the projection. Therefore (9.3) has been more accurately identified as the homotopy pushout

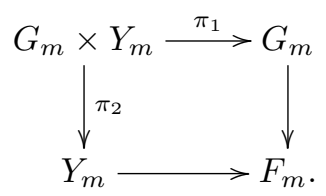

Hence, Lemma 6.1 immediately implies the following.

Lemma 9.2: There is an integral homotopy equivalence

$$
F_{m} \simeq G_{m} * Y_{m} .
$$

We take this a step further. Consider the homotopy fibration

$$
F_{m} \longrightarrow(\underline{X}, \underline{A})^{K} \longrightarrow(\underline{X}, \underline{A})^{K \backslash\{m\}} \times X_{m} .
$$

Since $K \backslash\{m\}$ and $\{m\}$ are the full subcomplexes of $K$ on the vertex sets $[m-1]$ and $\{m\}$ respectively, by Proposition 3.6, the polyhedral products $(\underline{X}, \underline{A})^{K \backslash\{m\}}$ and $(\underline{X}, \underline{A})^{\{m\}}=X_{m}$ both retract off $(\underline{X}, \underline{A})^{K}$. In general, these retractions cannot be multiplied together in $(\underline{X}, \underline{A})^{K}$, nor multiplied with the image of the map from $F_{m}$, because $(\underline{X}, \underline{A})^{K}$ is rarely an $H$-space. But after looping this can be done. Thus, if $\mu$ is the loop multiplication on $(\underline{X}, \underline{A})^{K}$, then the composite

$$
\begin{array}{r}
\Omega X_{m} \times \Omega(\underline{X}, \underline{A})^{K \backslash\{m\}} \times \Omega F_{m} \longrightarrow \Omega(\underline{X}, \underline{A})^{K} \times \Omega(\underline{X}, \underline{A})^{K} \times \Omega(\underline{X}, \underline{A})^{K} \\
\stackrel{\mu}{\longrightarrow} \Omega(\underline{X}, \underline{A})^{K}
\end{array}
$$


is a homotopy equivalence. Combining this with Lemma 9.2, we obtain the following.

Proposition 9.3: Let $K$ be a simplicial complex on the index set $[m]$. Then there is an integral homotopy equivalence

$$
\Omega(\underline{X}, \underline{A})^{K} \simeq \Omega X_{m} \times \Omega(\underline{X}, \underline{A})^{K \backslash\{m\}} \times \Omega\left(G_{m} * Y_{m}\right) .
$$

Since $K \backslash\{m\}$ is a simplicial complex on the index set [m-1], Proposition 9.3 can be applied again to decompose $\Omega(\underline{X}, \underline{A})^{K \backslash\{m\}}$. Iterating, for $1 \leq i \leq m$, let $R_{m-i}$ be the restriction of $K$ to the index set $[m-i]$. Note that $R_{m}=K$. For $1 \leq i<m$, let $L_{m-i}=\operatorname{link}_{R_{m-i+1}}(m-i+1)$. Then there is a star-link-restriction pushout of simplicial complexes

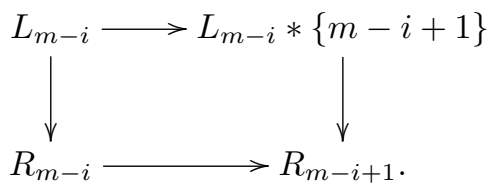

Let $G_{m-i}$ be the homotopy fibre of $(\underline{X}, \underline{A})^{L_{m-i}} \longrightarrow(\underline{X}, \underline{A})^{R_{m-i}}$, and let $Y_{m-i}$ be the homotopy fibre of the inclusion $A_{m-i} \longrightarrow X_{m-i}$. Then iterating Proposition 9.3 gives the following.

Theorem 9.4: Let $K$ be a simplicial complex on the vertex set $[m]$. Then there is an integral homotopy equivalence

$$
\Omega(\underline{X}, \underline{A})^{K} \simeq\left(\prod_{i=1}^{m} \Omega X_{i}\right) \times\left(\prod_{i=1}^{m} \Omega\left(G_{i} * Y_{i}\right)\right) .
$$

Remark 9.5: Theorem 9.4 is completely general, and says that the homotopy type of $\Omega(\underline{X}, \underline{A})^{K}$ depends only on the spaces $X_{i}$, the homotopy fibres of the inclusions $A_{m} \longrightarrow X_{m}$ and the homotopy fibres of the link-restriction maps $(\underline{X}, \underline{A})^{L_{i}} \longrightarrow(\underline{X}, \underline{A})^{R_{i}}$. So again we see the importance of knowing the homotopy class of the link-restriction maps.

Remark 9.6: Since $G_{i} * Y_{i} \simeq \Sigma G_{i} \wedge Y_{i}$, each of the factors $\Omega\left(G_{i} * Y_{i}\right)$ in the homotopy decomposition for $\Omega(\underline{X}, \underline{A})^{K}$ is a loop suspension. A great deal is known about the homotopy theory of loop suspensions. In particular, the Bott-Samelson Theorem states that if $R$ is a field then there is an algebra isomorphism $H_{*}(\Omega \Sigma Y ; R) \cong T\left(\widetilde{H}_{*}(Y ; R)\right)$, where $T(\cdot)$ is the free tensor 
algebra functor. Thus, from Theorem 9.4 and the Kunneth isomorphism, there is an algebra isomorphism

$$
H_{*}\left((\underline{X}, \underline{A})^{K} ; R\right) \cong\left(\bigotimes_{i=1}^{m} H_{*}\left(\Omega X_{i} ; R\right)\right) \otimes\left(\bigotimes_{i=1}^{m} T\left(\widetilde{H}_{*}\left(G_{i} \wedge Y_{i} ; R\right)\right)\right) .
$$

Also, James [25] shows that there is a homotopy equivalence

$$
\Sigma \Omega \Sigma Y \simeq \bigvee_{n=1}^{\infty} \Sigma Y^{(n)}
$$

where $Y^{(n)}$ is the $n$-fold smash product of $Y$ with itself. Thus, after suspending the homotopy equivalence for $\Omega(\underline{X}, \underline{A})^{K}$ and using James' result together with an iteration of the fact that $\Sigma(X \times Y) \simeq \Sigma X \vee \Sigma Y \vee(\Sigma X \wedge Y)$, we obtain a wedge decomposition for $\Sigma \Omega(\underline{X}, \underline{A})^{K}$ in terms of suspensions of iterated smash products of the spaces $\Omega X_{i}$ and $G_{i} \wedge Y_{i}$.

We mention two cases of Theorem 9.4. First, in the case $(\underline{X}, \underline{*})^{K}$, the homotopy fibre $Y_{i}$ of the inclusion $* \longrightarrow X_{i}$ is $\Omega X_{i}$. Thus we obtain the following.

Corollary 9.7: Let $K$ be a simplicial complex on the vertex set $[m]$. Then there is an integral homotopy equivalence

$$
\Omega(\underline{X}, \underline{*})^{K} \simeq\left(\prod_{i=1}^{m} \Omega X_{i}\right) \times\left(\prod_{i=1}^{m} \Omega\left(G_{i} * \Omega X_{i}\right)\right) .
$$

Second, in the case of $(\underline{C X}, \underline{X})$, since $C X_{i}$ is contractible, the term $\Omega C X_{i}$ in Theorem 9.4 is contractible, and the homotopy fibre of the inclusion $X_{i} \longrightarrow C X_{i}$ is homotopy equivalent to $X_{i}$. Thus we obtain the following.

Corollary 9.8: Let $K$ be a simplicial complex on the vertex set $[m]$. Then there is an integral homotopy equivalence

$$
\Omega(\underline{C X}, \underline{X})^{K} \simeq \prod_{i=1}^{m} \Omega\left(G_{i} * X_{i}\right) .
$$

As special cases of Corollaries 9.7 and 9.8 we obtain homotopy decompositions for the loops on the Davis-Januszkiewicz space and the loops on the moment-angle complex. In the first case, each pair $\left(X_{i}, *\right)$ is $\left(\mathbb{C} P^{\infty}, *\right)$, 
and $\Omega \mathbb{C} P^{\infty} \simeq S^{1}$. In the second case, each pair $\left(C X_{i}, X_{i}\right)$ is $\left(D^{2}, S^{1}\right)$. In particular, in the first case we have $G_{i} * \Omega X_{i} \simeq G_{i} * S^{1} \simeq \Sigma^{2} G_{i}$ and in the second case we have $G_{i} * X_{i} \simeq G_{i} * S^{1} \simeq \Sigma^{2} G_{i}$.

Corollary 9.9: Let $K$ be a simplicial complex on the vertex set $[m]$. Then there are integral homotopy equivalences

$$
\Omega D J_{K} \simeq\left(\prod_{i=1}^{m} S^{1}\right) \times\left(\prod_{i=1}^{m} \Omega \Sigma^{2} G_{i}\right)
$$

and

$$
\Omega \mathcal{Z}_{K} \simeq \prod_{i=1}^{m} \Omega \Sigma^{2} G_{i}
$$

Remark 9.10: Specializing Remark 9.6 to the case of $\Omega \mathcal{Z}_{K}$, for any field $R$ there is an algebra isomorphism

$$
H_{*}\left(\Omega \mathcal{Z}_{K} ; R\right) \cong \bigotimes_{i=1}^{m} T\left(\widetilde{H}_{*}\left(\Sigma G_{i} ; R\right)\right)
$$

and a homotopy equivalence for $\Sigma \Omega \mathcal{Z}_{K}$ in terms of suspensions of iterated smash products of the spaces $\Sigma G_{i}$.

\section{Exponents and Barratt's conjecture}

The homotopy decomposition in Theorem 9.4 is provocative. It is often the case in unstable homotopy theory that properties of a space $Y$ are studied by considering properties of $\Omega Y$. For example, if $Y$ is simply-connected then the homotopy groups of $\Omega Y$ are the same as those for $Y$ but shifted down one degree. One advantage of $\Omega Y$ over $Y$ is the loop multiplication, which better allows for the possibility of a homotopy decomposition into simpler factors. If $Y=\Sigma Y^{\prime}$ then the situation is greatly improved, as in Remark 9.6. Thus Theorem 9.4 suggests that there may be properties of $(\underline{X}, \underline{A})^{K}$ that may be better discerned by looking at $\Omega(\underline{X}, \underline{A})^{K}$.

In this section and Section 13 we describe applications of the loop space decomposition, although none is fully explored and many open questions remain. In this section we discuss a connection with homotopy exponents and Barratt's conjecture, and in Section 13 we discuss connections with rational homotopy theory and Moore's conjecture. To consider homotopy exponents we begin with some definitions. 
Definition 10.1: Let $X$ be a path-connected, pointed topological space and let $p$ be a prime. The homotopy exponent of $X$ at $p$ is the least power of $p$ that annihilates the $p$-torsion in $\pi_{*}(X)$.

If the power of $p$ is finite, say $r$, write $\exp _{p}(X)=p^{r}$. If there is no such least power of $p$, write $\exp _{p}(X)=\infty$.

Definition 10.2: Let $X$ be a path-connected co- $H$-space and let $p$ be a prime. The space $X$ has co-H-exponent $p^{r}$ if the degree $p^{r}$ map on $X$ is null homotopic but the degree $p^{r-1}$ map is not.

For example, for $m \geq 2$, let $P^{m}\left(p^{r}\right)$ be the Moore space, defined by the homotopy cofibration

$$
S^{m-1} \stackrel{p^{r}}{\longrightarrow} S^{m-1} \longrightarrow P^{m}\left(p^{r}\right) .
$$

Observe that if $m \geq 3$ then $P^{m}\left(p^{r}\right) \simeq \Sigma P^{m-1}\left(p^{r}\right)$, implying that $P^{m}\left(p^{r}\right)$ is a co- $H$-space. By [32] the degree $p^{r}$ map on $P^{m}\left(p^{r}\right)$ is null homotopic if $p^{r} \neq 2$, and if $p^{r}=2$ then the degree 4 map on $P^{m}(2)$ is null homotopic but the degree 2 map is not. Therefore the co- $H$-exponent of $P^{m}\left(p^{r}\right)$ is $p^{r}$ if $p^{r} \neq 2$, and is 4 if $p^{r}=2$.

Definition 10.3: Let $Y$ be a path-connected $H$-space and let $p$ be a prime. The space $Y$ has $H$-exponent $p^{r}$ if the $p^{r}$-power map on $Y$ is null homotopic but the $p^{r-1}$-power map is not.

Note that if $Y$ has $H$-exponent $p^{r}$ then $\exp _{p}(Y) \leq p^{r}$. For the $p^{r}$-power map on $Y$ induces multiplication by $p^{r}$ on homotopy groups. However, if $X$ has co- $H$-exponent $p^{r}$ then the $p^{r}$-power map on $\Omega X$ may not be null homotopic. This is due to the nontrivial difference between the loops on the degree $p^{r}$ map and the $p^{r}$-power map; this difference exists by the distributivity law and may be difficult to control (see [9] for an excellent discussion of the distributivity law). Barratt [4] conjectured that, in fact, while the difference may be complex its effect on exponents is minimal.

Conjecture: (Barratt) Let $X$ be a space with co- $H$-exponent $p^{r}$. Then the $p^{r+1}$-power map on $\Omega^{2} \Sigma X$ is null homotopic.

Barratt's conjecture is known to be true only for certain Moore spaces. If $p$ is odd and $r \geq 1$ then the conjecture holds by [33]. If $p=2$ and $r \geq 6$ the conjecture holds by [38]. For $p=2$ and $2 \leq r \leq 5$, upper bounds on the homotopy exponent of $P^{m}\left(2^{r}\right)$ are known [38]. The most interesting case is $P^{m}(2)$. Barratt's conjecture predicts that $\exp _{2}\left(P^{m}(2)\right)=8$. However, 
there is no known upper bound on the homotopy exponent of $P^{m}(2)$. That is, as far as anyone knows, there could be torsion of arbitrarily high order in $\pi_{*}\left(P^{m}(2)\right)$.

We will use polyhedral products to extend the range of spaces to which a positive resolution of Barratt's conjecture would apply.

Proposition 10.4: Let $K$ be a simplicial complex on the vertex set $[m]$ and let $p$ be a prime. Let $X_{1}, \ldots, X_{m}$ be co-H-spaces with co-H-exponent bounded above by $p^{r}$. If Barratt's conjecture holds, then the $p^{r+1}$-power map on $\Omega^{2}(\underline{X}, \underline{A})^{K}$ is null homotopic, and consequently $\exp _{p}\left((\underline{X}, \underline{A})^{K}\right) \leq p^{r+1}$.

Proof: In general, if $X$ is a co- $H$-space and $Y$ is a space then $Y \wedge X$ is also a co- $H$-space with comultiplication given by the composite

$$
Y \wedge X \stackrel{1 \wedge \sigma}{\longrightarrow} Y \wedge(X \vee X) \stackrel{\simeq}{\longrightarrow}(Y \wedge X) \vee(Y \wedge X)
$$

where $\sigma$ is the co-multiplication on $X$. Therefore, if the degree $p^{r}$ map on $X$ is null homotopic then so is the degree $p^{r}$ map on $Y \wedge X$.

In our case, by Corollary 9.8 there is a homotopy equivalence $\Omega(\underline{C X}, \underline{X})^{K} \simeq \prod_{i=1}^{m} \Omega\left(G_{i} * X_{i}\right)$. Looping, we obtain a homotopy equivalence of $H$-spaces $\Omega^{2}(\underline{C X}, \underline{X})^{K} \simeq \prod_{i=1}^{m} \Omega^{2}\left(G_{i} * X_{i}\right)$. Therefore the $p^{r+1}$ power map on $\Omega^{2}(\underline{C X}, \underline{X})^{K}$ is null homotopic if and only if the same is true for each factor $\Omega^{2}\left(G_{i} * X_{i}\right)$. Since each co- $H$-space $X_{i}$ has co- $H$-exponent at most $p^{r}$, the same is therefore true for each $G_{i} \wedge X_{i}$. So Barratt's conjecture implies that the $p^{r+1}$-power map on $\Omega^{2}\left(G_{i} * X_{i}\right) \simeq \Omega^{2}\left(\Sigma G_{i} \wedge X_{i}\right)$ is null homotopic.

Since Barratt's conjecture holds for Moore spaces $P^{m}\left(p^{r}\right)$ where $p$ is odd or where $p=2$ and $r \geq 6$, Proposition 10.4 also holds in these cases.

\section{Elliptic and hyperbolic spaces}

Félix, Halperin and Thomas [12] proved the remarkable fact that a finite dimensional path-connected, pointed space $X$ either has finitely many rational homotopy groups or the total number of rational homotopy groups in dimensions $m \leq n$ grows exponentially with $n$. That is, there is no space whose rational homotopy groups have polynomial growth. Plainly put, either $X$ has very few rational homotopy groups or it has an awful lot. There's no middle ground.

It is natural to ask which spaces fall into which category. It helps to give the two options names. 
Definition 11.1: A finite dimensional space $X$ is elliptic if it has finitely many rational homotopy groups. Otherwise $X$ is hyperbolic.

The canonical example of an elliptic space is a product of spheres. The canonical example of a hyperbolic space is a wedge of two spheres (hyperbolicity is proved using the Hilton-Milnor Theorem). In what follows we will give a simple way of identifying a large number of hyperbolic polyhedral products.

Observe that if $K \neq \Delta^{m-1}$ then it has a missing face, and so it has at least one minimal missing face.

Lemma 11.2: Let $K$ be a simplicial complex on the vertex set $[m]$ and suppose that $K \neq \Delta^{m-1}$. Let $\sigma=\left(i_{1}, \ldots, i_{k}\right) \in M M F(K)$. Then $\Omega X_{i_{1}} *$ $\cdots * \Omega X_{i_{k}}$ is an integral retract of $(\underline{C \Omega X}, \underline{\Omega X})^{K}$ and $\Omega\left(\Omega X_{i_{1}} * \cdots * \Omega X_{i_{k}}\right)$ is an integral retract of $\Omega(\underline{X}, \underline{*})^{K}$.

Proof: Since $\sigma=\left(i_{1}, \ldots, i_{k}\right)$ is a minimal missing face of $K$, we have $\sigma \notin K$ but $\partial \sigma \subseteq K$. In particular, the full subcomplex of $K$ on the vertex set $I=\left\{i_{1}, \ldots, i_{k}\right\}$ is $K_{I}=\partial \sigma$. So by Proposition 3.6, $(\underline{C \Omega X}, \underline{\Omega X})^{K_{I}}$ retracts off of $(\underline{C \Omega X}, \underline{\Omega X})^{K}$. On the other hand, by Lemma 8.7, there is an integral homotopy equivalence $(\underline{C \Omega X}, \underline{\Omega X})^{K_{I}}=(\underline{C \Omega X}, \underline{\Omega X})^{\partial \sigma} \simeq \Omega X_{i_{1}} * \cdots * \Omega X_{i_{k}}$. Thus $\Omega X_{i_{1}} * \cdots * \Omega X_{i_{k}}$ is a retract of $(\underline{C \Omega X}, \underline{\Omega X})^{K}$.

By Lemma 3.16, there is an integral homotopy equivalence $\Omega(\underline{X}, \underline{*})^{K} \simeq$ $\prod_{i=1}^{m} \Omega X_{i} \times \Omega(\underline{C \Omega X}, \underline{\Omega X})^{K}$. Therefore the second assertion of the lemma follows from the first.

Theorem 11.3: Let $K$ be a simplicial complex on the vertex set $[m]$ and suppose that $K \neq \Delta^{m-1}$. Let $\sigma=\left(i_{1}, \ldots, i_{k}\right) \in M M F(K)$. Suppose that $(\underline{X}, \underline{*})$ is a sequence of pointed $C W$-pairs where each $X_{i}$ is simply-connected and rationally nontrivial, and suppose that the rank of $\widetilde{H}_{*}\left(\Omega X_{i_{j}} ; \mathbb{Q}\right)$ is at least 2 for some $1 \leq j \leq k$. Then $(\underline{X}, \underline{*})^{K}$ is hyperbolic.

Proof: To show that a simply-connected space $Y$ is hyperbolic it is equivalent to show that $\Omega Y$ is hyperbolic. By Lemma 3.16, there is a homotopy equivalence $\Omega(\underline{X}, \underline{*})^{K} \simeq \prod_{i=1}^{m} \Omega X_{i} \times \Omega(\underline{C \Omega X}, \underline{\Omega X})^{K}$. So to show that $(\underline{X}, \underline{*})^{K}$ is hyperbolic it suffices to show that $(\underline{C \Omega X}, \underline{\Omega X})^{K}$ is hyperbolic. Further, by Lemma $11.2, \Omega X_{i_{1}} * \cdots * \Omega X_{i_{k}}$ is a retract of $(\underline{C \Omega X}, \underline{\Omega X})^{K}$, so it suffices to show that $\Omega X_{i_{1}} * \cdots * \Omega X_{i_{k}}$ is hyperbolic.

By hypothesis, each $X_{i_{j}}$ is simply-connected and rationally nontrivial, so the rank of $\widetilde{H}_{*}\left(\Omega X_{i_{j}} ; \mathbb{Q}\right)$ is at least 1 for each $1 \leq j \leq k$. Therefore, 
as at least one of these ranks must be strictly greater than 1 , the rank of $\widetilde{H}_{*}\left(\Omega X_{i_{1}} * \cdots * \Omega X_{i_{k}} ; \mathbb{Q}\right)$ is at least 2 . In general, a co- $H$-space is rationally homotopy equivalent to a wedge of spheres. As $\Omega X_{i_{1}} * \cdots * \Omega X_{i_{j}} \simeq$ $\Sigma^{k-1} \Omega X_{i_{1}} \wedge \cdots \wedge \Omega X_{i_{k}}$, it follows that $\Omega X_{i_{1}} * \cdots * \Omega X_{i_{k}}$ is rationally homotopy equivalent to a wedge of spheres. As the rational homology of this space has rank at least $2, \Omega X_{i_{1}} * \cdots * \Omega X_{i_{k}}$ is rationally homotopy equivalent to a wedge of at least 2 spheres. But a wedge of 2 or more spheres is hyperbolic, and so $\Omega X_{i_{1}} * \cdots * \Omega X_{i_{k}}$ is hyperbolic.

Theorem 11.3 is a mild generalization of a result by Félix and Tanré [13], which considered the case when each $X_{i}$ equals a common space $X$. However, the methods are very different. Félix and Tanré used a Sullivan minimal model argument which gives only rational information. The proof of Theorem 11.3 used a minimal missing face argument which gives integral information, from which rational information can be deduced. This distinction will be important in the next section when we consider Moore's conjecture.

Theorem 11.3 implies that if $(\underline{X}, \underline{*})^{K}$ is elliptic for $K \neq \Delta^{m-1}$ then each $\Omega X_{i}$ must have at most one generator in reduced rational homology. That is, each $\Omega X_{i}$ is either rationally contractible or rationally a sphere. So while Theorem 11.3 identifies many hyperbolic spaces, it does not apply at all to the case of $D J_{K}=\left(\mathbb{C} P^{\infty}, *\right)^{K}$ since each $\Omega X_{i}$ equals $\Omega \mathbb{C} P^{\infty} \simeq$ $S^{1}$. Bahri, Bendersky, Cohen and Gitler [2] considered the case of DavisJanuszkiewicz spaces and showed that $D J_{K}$ is elliptic if and only if it is a product of spheres and discs.

\section{Moore's conjecture}

There is a major conjecture in homotopy theory which relates the notion of a space being elliptic or hyperbolic with homotopy exponents.

Conjecture: Moore Let $X$ be a finite $C W$-complex. Then the following are equivalent:

(a) $X$ is elliptic;

(b) $X$ has a finite homotopy exponent at every prime $p$;

(c) $X$ has a finite homotopy exponent at some prime $p$.

Moore's conjecture is known to hold in a limited number of cases. Elliptic spaces with finite exponents at all primes include spheres $[26,41]$, finite $H$-spaces [28], $H$-spaces with finitely generated cohomology [8], and 
odd primary Moore spaces [33]. Hyperbolic spaces with no exponent at any prime include $S^{n} \vee S^{m}$ by the Hilton-Milnor Theorem, torsion-free suspensions [36], and two-cell complexes [34]. There are also partial results. In [31] it was shown that if $X$ is elliptic then it has an exponent at all but finitely many primes, and in [37] it was shown that if $X$ is hyperbolic and $H_{*}(\Omega X ; \mathbb{Z})$ is $p$-torsion free then, provided $p$ is large enough, $X$ has no exponent at $p$.

The methods used in Theorem 11.3 to show that $(\underline{X}, \underset{*}{*})^{K}$ is hyperbolic given certain conditions on the spaces $X_{i}$ do more. We will use them to given examples when Moore's conjecture can be resolved. The statement is about hyperbolic spaces, so Moore's conjecture takes the form: if a space $Y$ is hyperbolic then it has no exponent at $p$ for any prime $p$. In this context the proof of the following lemma is immediate.

Lemma 12.1: Given the hypotheses of Theorem 11.3, if there is a space $Y$ which retracts off $\Omega X_{i_{1}} * \cdots * \Omega X_{i_{k}}$ and $Y$ has no exponent at any prime $p$, then $(\underline{X}, \underline{*})^{K}$ has no exponent any prime $p$.

For example, if $S^{n_{1}} \vee S^{n_{2}}$ retracts off $\Omega X_{i_{1}} * \cdots * \Omega X_{i_{k}}$ then $(\underline{X}, \underline{*})^{K}$ has no exponent at any prime $p$. We give two examples where this occurs.

Example 12.2: Take $X_{i}=S^{n_{i}}$ for each $1 \leq i \leq m$, where $n_{i} \geq 2$, and consider the $C W$-pairs $\left(S^{n_{i}}, *\right)$. By [25], there is a homotopy equivalence

$$
\Sigma \Omega S^{n+1} \simeq \bigvee_{i=1}^{\infty} S^{n i+1}
$$

Iterating this homotopy equivalence implies that $\Omega S^{n_{i_{1}}} * \cdots * \Omega S^{n_{i_{k}}} \simeq$ $\Sigma^{k-1} \Omega S^{n_{i_{1}}} \wedge \cdots \wedge \Omega S^{n_{i_{k}}}$ is homotopy equivalent to an infinite wedge of spheres. Consequently, $\Omega S^{n_{i_{1}}} * \cdots * \Omega S^{n_{i_{k}}}$ has no exponent at any prime $p$ and so the corresponding polyhedral product $(\underline{X}, \underline{*})^{K}$ has no exponent at any prime $p$.

Example 12.3: Take $X_{i}=\mathbb{C} P^{n_{i}}$ for each $1 \leq i \leq m$, where $n_{i} \geq 1$, and consider the $C W$-pairs $\left(\mathbb{C} P^{n_{i}}, *\right)$. It is well known that there is a homotopy equivalence

$$
\Omega \mathbb{C} P^{n} \simeq S^{1} \times \Omega S^{2 n+1} .
$$


Therefore, by Lemma 4.1 and (12.1), there are homotopy equivalences

$$
\begin{aligned}
\Sigma \Omega \mathbb{C} P^{n} & \simeq \Sigma\left(S^{1} \times \Omega S^{2 n+1}\right) \\
& \simeq \Sigma S^{1} \vee \Sigma \Omega S^{2 n+1} \vee\left(\Sigma S^{1} \wedge \Omega S^{2 n+1}\right) \\
& \simeq S^{2} \vee\left(\bigvee_{i=1}^{\infty} S^{2 n i+1}\right) \vee\left(\bigvee_{j=1}^{\infty} S^{2 n i+2}\right)
\end{aligned}
$$

Iterating this homotopy decomposition, $\Omega \mathbb{C} P^{n_{i_{1}}} * \cdots * \Omega \mathbb{C} P^{n_{i_{k}}}$ is homotopy equivalent to an infinite wedge of spheres, and so has no exponent at any prime $p$. Therefore the corresponding polyhedral product $(\underline{X}, \underline{*})^{K}$ also has no exponent at any prime $p$.

\section{More on elliptic and hyperbolic spaces, and Moore's conjecture}

In this section we use the homotopy equivalence

$$
\Omega(\underline{X}, \underline{A})^{K} \simeq\left(\prod_{i=1}^{m} \Omega X_{i}\right) \times\left(\prod_{i=1}^{m} \Omega\left(G_{i} * Y_{i}\right)\right)
$$

in Theorem 9.4 as a tool to further investigate whether polyhedral products are elliptic or hyperbolic, and whether Moore's conjecture holds. Two initial observations are as follows.

Lemma 13.1: Let $K$ be a simplicial complex on the vertex set $[m]$ and let $(\underline{X}, \underline{A})$ be a sequence of pointed $C W$-pairs. Then the following hold:

(a) if any one of $X_{1}, \ldots, X_{m}$ is hyperbolic then so is $(\underline{X}, \underline{A})^{K}$;

(b) if any $G_{i} \wedge Y_{i}$ is rationally nontrivial and has the property that one of $G_{i}$ or $Y_{i}$ has two or more generators in rational homology then $(\underline{X}, \underline{A})^{K}$ is hyperbolic.

Proof: Part (a) follows immediately since each $\Omega X_{i}$ is a retract of $\Omega(\underline{X}, \underline{A})^{K}$, implying that the rational homotopy groups of $X_{i}$ retract off those of $(\underline{X}, \underline{A})^{K}$.

For part (b), in general, if $X$ is a co- $H$-space with $n$ generators in rational homology then $X$ is rationally equivalent to a wedge of spheres, one for each generator. In our case, each $G_{i} * Y_{i} \simeq \Sigma G_{i} \wedge Y_{i}$ is a co- $H$-space and so is rationally equivalent to a wedge of spheres. Rationally, there is a Kunneth isomorphism, so

$$
\widetilde{H}_{*}\left(G_{i} \wedge Y_{i} ; \mathbb{Q}\right) \cong \widetilde{H}_{*}\left(G_{i} ; \mathbb{Q}\right) \otimes \widetilde{H}_{*}\left(Y_{i} ; \mathbb{Q}\right) .
$$


By hypothesis, $G_{i} \wedge Y_{i}$ is rationally nontrivial, which implies that there is some nontrivial class in $\widetilde{H}_{*}\left(G_{i} \wedge Y_{i} ; \mathbb{Q}\right)$. The Kunneth isomorphism therefore implies that there is at least one nontrivial class in each of $\widetilde{H}_{*}\left(G_{i} ; \mathbb{Q}\right)$ and $\widetilde{H}_{*}\left(Y_{i} ; \mathbb{Q}\right)$. Thus if at least one of $\widetilde{H}_{*}\left(G_{i} ; \mathbb{Q}\right)$ or $\widetilde{H}_{*}\left(Y_{i} ; \mathbb{Q}\right)$ has two or more generators then so does $\widetilde{H}_{*}\left(G_{i} \wedge Y_{i} ; \mathbb{Q}\right)$. The same is then true for $\widetilde{H}_{*}\left(\Sigma G_{i} \wedge Y_{i} ; \mathbb{Q}\right)$, and so $\Sigma G_{i} \wedge Y_{i}$ is homotopy equivalent to a wedge of at least two spheres, implying that it is hyperbolic.

Lemma 13.1 indicates that the property of being elliptic is pretty restrictive. If $(\underline{X}, \underline{A})^{K}$ is elliptic then each of $X_{1}, \ldots, X_{m}$ must be elliptic, and no $G_{i} \wedge Y_{i}$ can have more than one rational homology class. To have one rational homology class implies that $G_{i} \wedge Y_{i}$ is rationally a sphere, which further implies that both $G_{i}$ and $Y_{i}$ are rationally spheres. This restriction on $G_{i}$, which is the homotopy fibre of a link into a restriction, is particularly severe.

We will give a more explicit description of a large family of hyperbolic spaces in Propositions 13.7 and 13.10. The idea is to relate the minimal missing faces of $\operatorname{link}_{K}(v)$ to $K \backslash\{v\}$ in order to identify nontrivial rational homotopy classes in one of the spaces $G_{i}$. Recall from Section 8 that $\operatorname{MMF}(K)$ is the set of minimal missing faces of $K$.

Lemma 13.2: Let $K$ and $L$ be simplicial complexes on the same vertex set $[m]$ and suppose that $K$ is a subcomplex of $L$. If $M M F(K) \subseteq M M F(L)$ then $M M F(K)=\operatorname{MMF}(L)$.

Proof: We aim for a contradiction. Suppose that $\sigma=\left(i_{1}, \ldots, i_{k}\right) \in$ $\operatorname{MMF}(L)$ but $\sigma \notin \operatorname{MMF}(K)$. Since $\sigma$ is a minimial missing face of $L$, by definition we have $\partial \sigma \subset L$. This implies that every proper face of $\sigma$ is a face of $L$. On the other hand, since $\sigma$ is a missing face of $L$ it is also a missing face of the subcomplex $K$. Therefore some proper face $\tau$ of $\sigma$ must be in $\operatorname{MMF}(K)$. By hypothesis, $\operatorname{MMF}(K) \subseteq \operatorname{MMF}(L)$, implying that $\tau \in \operatorname{MMF}(L)$. But we have already said that every proper face of $\sigma$ is a face of $L$, and so cannot be a missing face, and therefore not in $\operatorname{MMF}(L)$. This is a contradiction.

Remark 13.3: In Lemma 13.2 it is important that $K$ and $L$ have the same vertex set. For if $K$ is two disjoint vertices $v_{1}$ and $v_{2}$, and $L$ is three disjoint vertices $v_{1}, v_{2}$ and $v_{3}$ then $\left(v_{2}, v_{3}\right) \in \operatorname{MMF}(L)$ but it is not in $\operatorname{MMF}(K)$ since 3 is not even a vertex of $K$. 
Lemma 13.4: Let $K$ and $L$ be simplicial complexes on the same vertex set $[m]$ and suppose that $K$ is a subcomplex of $L$. If $M M F(K)=M M F(L)$ then $K=L$.

Proof: Suppose that $K \neq L$. Then there is a face $\sigma \in L$ such that $\sigma \notin K$. Therefore $\sigma$ is a missing face of $K$, and so either $\sigma \in \operatorname{MMF}(K)$ or some proper face $\tau$ of $\sigma$ is in $\operatorname{MMF}(K)$. If $\sigma \in \operatorname{MMF}(K)$ then by hypothesis $\sigma \in \operatorname{MMF}(L)$. But then $\sigma$ is a missing face of $L$, a contradiction. If $\tau \in$ $\operatorname{MMF}(K)$ for some proper face $\tau$ of $\sigma$ then, by hypothesis, $\tau \in \operatorname{MMF}(L)$ as well. But then $\tau$ is a missing face of $L$, implying that $\sigma$ is a missing face of $L$, a contradiction.

Suppose that $K$ is a subcomplex of $L$. A minimal missing face $\sigma$ of $K$ is said to be filled in inside $L$ if $\sigma \in L$. Specialize to the case of $(\underline{X}, \underline{*})$.

Lemma 13.5: Let $K$ be a simplicial complex on the vertex set $[m]$. Let $v$ be a vertex of $K$. Then precisely one of the following hold:

(a) $\operatorname{link}_{K}(v)$ is a full subcomplex of $K \backslash\{v\}$;

(b) there is a minimal missing face in $\operatorname{link}_{K}(v)$ that is filled in inside $K \backslash\{v\}$.

Proof: Suppose that $\operatorname{link}_{K}(v)$ is not a full subcomplex of $K \backslash\{v\}$. Then it must be shown that $\operatorname{link}_{K}(v)$ has a minimal missing face that is filled in inside $K \backslash\{v\}$. First we refine the problem. Let $I$ be the vertex set of $\operatorname{link}_{K}(v)$. This may be a proper subset of the vertex set of $K \backslash\{v\}$. So let $R(v)$ be the full subcomplex of $K \backslash\{v\}$ on the vertex set $I$. Observe that a minimal missing face of $\sigma$ is filled in inside $K \backslash\{v\}$ if and only if it is filled in inside $R(v)$. So it is equivalent to show that $\operatorname{link}_{K}(v)$ has a minimal missing face that is filled in inside $R(v)$.

Suppose not. Then every minimal missing face $\sigma$ of $\operatorname{link}_{K}(v)$ is also a missing face of $R(v)$. But then $\sigma$ is a minimal missing face of $R(v)$, for every proper face of $\sigma$ is in $\operatorname{link}_{K}(v)$ and so must be in $R(v) \operatorname{since} \operatorname{link}_{K}(v)$ is a subcomplex. Therefore $\operatorname{MMF}\left(\operatorname{link}_{K}(v)\right) \subseteq \operatorname{MMF}(R(v))$. By definition of $R(v)$, both $R(v)$ and $\operatorname{link}_{K}(v)$ have the same vertex set, so by Lemma 13.2 $\operatorname{MMF}\left(\operatorname{link}_{K}(v)\right)=\operatorname{MMF}(R(v))$, and therefore by Lemma 13.4, $\operatorname{link}_{K}(v)=$ $R(v)$. That is, $\operatorname{link}_{K}(v)$ is a full subcomplex of $K \backslash\{v\}$, a contradiction.

Guided by the two options in Lemma 13.5, we consider conditions that guarantee that certain polyhedral products are hyperbolic by studying how 
the combinatorics of $K$ influence the homotopy type of the homotopy fibre of the inclusion of $(\underline{X}, \underline{A})^{\operatorname{link}_{K}(v)}$ into $(\underline{X}, \underline{A})^{K \backslash\{v\}}$. Let $v$ be a vertex of $K$. Define the space $G(v)$ and the map $g(v)$ by the homotopy fibration

$$
G(v) \stackrel{g(v)}{\longrightarrow}(\underline{X}, \underline{A})^{\operatorname{link}_{K}(v)} \longrightarrow(\underline{X}, \underline{A})^{K \backslash\{v\}} .
$$

Lemma 13.6: Let $K$ be a simplicial complex on the vertex set $[m]$. Suppose that $v$ is a vertex of $K$ such that $\operatorname{lin}_{K}(v)$ is a full subcomplex of $K \backslash\{v\}$. Then the map $g(v)$ is null homotopic, implying that there is a homotopy equivalence

$$
\Omega(\underline{X}, \underline{A})^{K \backslash\{v\}} \simeq \Omega(\underline{X}, \underline{A})^{l i n k_{K}(v)} \times G(v) .
$$

Further, if $\operatorname{link}_{K}(v)$ is a proper full subcomplex of $K \backslash\{v\}$ and $u_{j_{1}}, \ldots, u_{j_{\ell}}$ are vertices of $K \backslash\{v\}$ but not of $\operatorname{link}_{K}(v)$, then $\prod_{t=1}^{\ell} \Omega X_{j_{t}}$ retracts off $G(v)$.

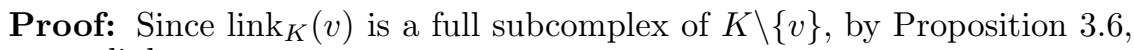
$(\underline{X}, \underline{A})^{\operatorname{link}_{K}(v)}$ is a retract of $(\underline{X}, \underline{A})^{K \backslash\{v\}}$. Therefore the map $g(v)$ is null homotopic, and the asserted homotopy equivalence for $\Omega(\underline{X}, \underline{A})^{K \backslash\{v\}}$ follows immediately.

For the second statement, let $u$ be a vertex of $K \backslash\{v\}$ that is not a vertex of $\operatorname{link}_{K}(v)$. The full subcomplex of $K \backslash\{v\}$ on the vertex set $\{u\}$ is just the single vertex $u$. So by Proposition 3.6, $(\underline{X}, \underline{A})^{\{u\}}$ retracts off of $(\underline{X}, \underline{A})^{K \backslash\{v\}}$. But by definition of the polyhedral product, $(\underline{X}, \underline{A})^{\{u\}}=X_{u}$. Therefore, $X_{u}$ retracts off $(\underline{X}, \underline{A})^{K \backslash\{v\}}$, but it cannot retract off of $(\underline{X}, \underline{A})^{\operatorname{link}_{K}(v)}$ since this is a subspace of $\prod_{\substack{i=1 \\ i \neq u, v}}^{m} X_{i}$. Thus $\Omega X_{u}$ is a factor of $\Omega(\underline{X}, \underline{A})^{K \backslash\{v\}}$ but not a factor of $\Omega(\underline{X}, \underline{A})^{\operatorname{link}_{K}(v)}$, implying that $\Omega X_{u}$ is a factor of $G(v)$. As this holds for each of the vertices $u_{j_{1}}, \ldots, u_{j_{\ell}}$, the product $\prod_{t=1}^{\ell} \Omega X_{j_{t}}$ retracts off $G(v)$.

Proposition 13.7: Let $K$ be a simplicial complex on the vertex set $[\mathrm{m}]$. Suppose that $v$ is a vertex of $K$ such that $\operatorname{link}_{K}(v)$ is a full subcomplex of $K \backslash\{v\}$, and suppose that $u_{j_{1}}, \ldots, u_{j_{\ell}}$ are vertices of $K \backslash\{v\}$ that are not in $\operatorname{link}_{K}(v)$. If $X_{1}, \ldots, X_{m}$ are pointed, simply-connected $C W$-complexes such that the rank of $\widetilde{H}_{*}\left(\prod_{t=1}^{\ell} \Omega X_{j_{t}} ; \mathbb{Q}\right)$ is at least 2 and $\Omega X_{v}$ is rationally nontrivial then $(\underline{X}, \underline{*})^{K}$ is hyperbolic.

Proof: By Lemma 13.6, $\prod_{t=1}^{\ell} \Omega X_{j_{t}}$ retracts off $G(v)$. So if the rank of $\widetilde{H}_{*}\left(\prod_{t=1}^{\ell} \Omega X_{j_{t}} ; \mathbb{Q}\right)$ is at least 2 then the rank of $\widetilde{H}_{*}(G(v) ; \mathbb{Q})$ is at least 2 . 
Therefore, as $\Omega X_{v}$ is rationally nontrivial, the rank of $\widetilde{H}_{*}\left(G(v) * \Omega X_{v} ; \mathbb{Q}\right)$ is at least 2 . Since $G(v) * \Omega X_{v}$ is a suspension, it is rationally homotopy equivalent to a wedge of spheres, and as the space has rank at least 2 in rational homology, this wedge is of at least 2 spheres. Therefore $G(v) * \Omega X_{v}$ is hyperbolic. By Theorem 9.4, $\Omega\left(G(v) * \Omega X_{v}\right)$ retracts off $\Omega(\underline{X}, *)^{K}$, implying that $\Omega(\underline{X}, \underline{*})^{K}$ is hyperbolic.

Remark 13.8: The proof of Proposition 13.7 implies more. Since $\prod_{t=1}^{\ell} \Omega X_{j_{t}}$ retracts off $G(v)$, there is a retraction of $\Sigma\left(\prod_{t=1}^{\ell} \Omega X_{j_{t}}\right) \wedge \Omega X_{v}$ off of $G(v) * \Omega X_{v}$. So if a wedge of spheres retracts off $\Sigma\left(\prod_{t=1}^{\bar{\ell}} \Omega X_{j_{t}}\right) \wedge \Omega X_{v}$ then $G(v) * \Omega X_{v}$ has no exponent at any prime $p$ and therefore neither does $(\underline{X}, \underline{*})^{K}$, implying that Moore's conjecture holds in this case.

The second case is when, for some vertex $v$ of $K$, there is a minimal missing face of $\operatorname{link}_{K}(v)$ that is filled in inside $K \backslash\{v\}$. For this case we specialize to $(\underline{C X}, \underline{X})$ so that a product of cones, which is contractible, shows up at the right point. By definition, if $\sigma=\left(i_{1}, \ldots, i_{k}\right)$ is a minimal missing face of $\operatorname{link}_{K}(v)$ then $\partial \sigma \subseteq \operatorname{link}_{K}(v)$. The full subcomplex of $\operatorname{link}_{K}(v)$ on the vertex set $\left\{i_{1}, \ldots, i_{k}\right\}$ is therefore $\partial \sigma$. Therefore, by Proposition 3.6, $(\underline{C X}, \underline{X})^{\partial \sigma}$ is a retract of $(\underline{C X}, \underline{X})^{\operatorname{link}_{K}(v)}$. Since $\partial \sigma$ is the boundary of a simplex, by Lemma 8.7, $(\underline{C X}, \underline{X})^{\partial \sigma} \simeq X_{i_{1}} * \cdots * X_{i_{k}}$.

Lemma 13.9: Let $K$ be a simplicial complex on the vertex set $[m]$. Suppose that $v$ is a vertex of $K$ such that there is a minimal missing face $\sigma=$ $\left(i_{1}, \ldots, i_{k}\right)$ of $\operatorname{link}_{K}(v)$ that is filled in inside $K \backslash\{v\}$. Then there is a lift

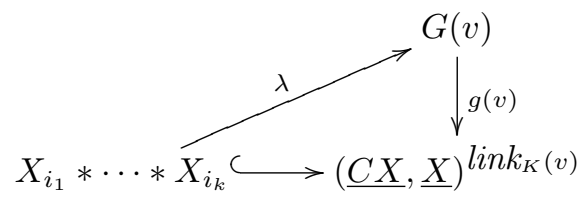

for some map $\lambda$ which has a left homotopy inverse.

Proof: Since $\sigma$ is a minimal missing face of $\operatorname{link}_{K}(v)$ we have $\partial \sigma \subseteq$ $\operatorname{link}_{K}(v)$. Since $\sigma$ is filled in inside $K \backslash\{v\}$ there is a commutative diagram of simplicial complexes

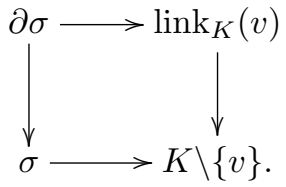


Now take polyhedral products with respect to $(\underline{C X}, \underline{X})$ to obtain a commutative diagram of polyhedral products

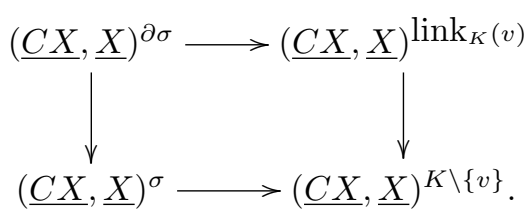

As mentioned prior to the lemma, $(\underline{C X}, \underline{X})^{\partial \sigma} \simeq X_{i_{1}} * \cdots * X_{i_{k}}$. Since $\sigma$ is a simplex, by Example $2.3(\underline{C X}, \underline{X})^{\sigma}=C X_{i_{1}} \times \cdots \times C X_{i_{k}}$, so $(\underline{C X}, \underline{X})^{\sigma}$ is contractible. Thus from (13.1) we obtain a homotopy commutative diagram

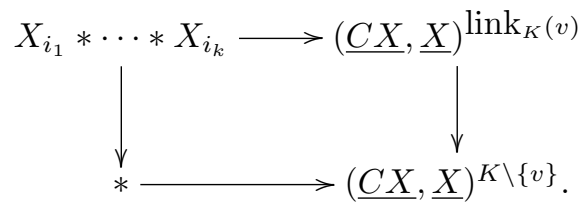

Therefore, as there is a homotopy fibration $G(v) \stackrel{g(v)}{\longrightarrow}(\underline{C X}, \underline{X})^{\operatorname{link}_{K}(v)} \longrightarrow$ $(\underline{C X}, \underline{X})^{K \backslash\{v\}}$, the map $X_{i_{1}} * \cdots * X_{i_{k}} \longrightarrow(\underline{C X}, \underline{X})^{\operatorname{link}_{K}(v)}$ lifts through $g(v)$ to a map $\lambda: X_{i_{1}} * \cdots * X_{i_{k}} \longrightarrow G(v)$. Since the map $X_{i_{1}} * \cdots *$ $X_{i_{k}} \longrightarrow(\underline{C X}, \underline{X})^{\operatorname{link}_{K}(v)}$ is induced by the inclusion of the full subcomplex $\partial \sigma$ into $\operatorname{link}_{K}(v)$, by Proposition 3.6 it has a left homotopy inverse $t:(\underline{C X}, \underline{X})^{\operatorname{link}_{K}(v)} \longrightarrow X_{i_{1}} * \cdots * X_{i_{k}}$. Therefore $t \circ g(v)$ is a left homotopy inverse of $\lambda$.

Proposition 13.10: Let $K$ be a simplicial complex on the vertex set $[\mathrm{m}]$. Suppose that $v$ is a vertex of $K$ such that there is a minimal missing face $\sigma=\left(i_{1}, \ldots, i_{k}\right)$ of $\operatorname{link}_{K}(v)$ that is filled in inside $K \backslash\{v\}$. Suppose that $X_{1}, \ldots, X_{m}$ are pointed, connected $C W$-complexes such that each $X_{i_{j}}$ for $1 \leq j \leq k$ and $X_{v}$ are rationally nontrivial, and at least one of these spaces has rank 2 in rational homology. Then $(\underline{C X}, \underline{X})^{K}$ is hyperbolic.

Proof: By Lemma 13.9, $X_{i_{1}} * \cdots * X_{i_{k}}$ retracts off $G(v)$, and so $X_{i_{1}} * \cdots *$ $X_{i_{k}} * X_{v}$ retracts off $G(v) * X_{v}$. The hypotheses on the spaces $X_{i_{j}}$ and $X_{v}$ imply that $X_{i_{1}} * \cdots * X_{i_{k}} * X_{v}$ is rationally nontrivial and has rank at least 2 in rational homology. Therefore $G(v) * X_{v}$ has rank at least 2 in rational homology. As $G(v) * X_{v}$ is a suspension, it is rationally homotopy equivalent to a wedge of spheres. The fact that $\widetilde{H}_{*}\left(G(v) * X_{v} ; \mathbb{Q}\right)$ has rank at least 2 implies that this wedge is of at least 2 spheres. Therefore $G(v) * X_{v}$ is hyperbolic. 
By Theorem $9.4, \Omega\left(G(v) * X_{v}\right)$ retracts off $\Omega(\underline{C X}, \underline{X})^{K}$, so as $G(v) * X_{v}$ is hyperbolic, so is $(\underline{C X}, \underline{X})^{K}$.

Remark 13.11: As in Remark 13.8, the proof of Proposition 13.10 shows that if a wedge of spheres retracts off of $G(v) * X_{v}$ then $(\underline{C X}, \underline{X})^{K}$ has no exponent at any prime $p$, implying that Moore's conjecture holds in this case.

Propositions 13.7 and 13.10 should be regarded as a starting point for investigating whether polyhedral products are elliptic or hyperbolic. A key feature is that this is done by first determining integral results, and then deducing the rational information about ellipticity or hyperbolicity. The integral results have the advantage that they are well suited to going further to address Moore's conjecture. Much remains to be done in this direction, and we close with some questions.

Problem 13.12: Determine which polyhedral products $(\underline{X}, \underline{*})^{K}$ or $(\underline{C X}, \underline{X})^{K}$ are elliptic and which are hyperbolic.

One way forward in Problem 13.12 is to look for two rational classes in $\widetilde{H}_{*}\left(G(v) * \Omega X_{v} ; \mathbb{Q}\right.$ ) (or $\widetilde{H}_{*}\left(G(v) * X_{v} ; \mathbb{Q}\right)$ ) not by looking for one nontrivial class in $\widetilde{H}_{*}(G(v) ; \mathbb{Q})$ and two in $\widetilde{H}_{*}\left(\Omega X_{v} ; \mathbb{Q}\right)\left(\right.$ or $\left.\widetilde{H}_{*}\left(X_{v} ; \mathbb{Q}\right)\right)$ but to look for two nontrivial classes in $\widetilde{H}_{*}(G(v) ; \mathbb{Q})$ and one in $\widetilde{H}_{*}\left(\Omega X_{v} ; \mathbb{Q}\right)$ (or $\widetilde{H}_{*}\left(X_{v} ; \mathbb{Q}\right)$ ). For example, if $\operatorname{link}_{K}(v)$ has at least two minimal missing faces filled in inside $K \backslash\{v\}$ then Lemma 13.9 gives two retractions off $G(v)$, and these are independent since they correspond to independent full subcomplex retractions off of $(\underline{C X}, \underline{X})^{\operatorname{link}_{K}(v)}$. So one is left having to deal with the cases where, for every vertex $v$ in $K, \operatorname{link}_{K}(v)$ has 0 or 1 minimal missing faces filled in inside $K \backslash\{v\}$.

This perspective is particularly important because Propositions 13.7 and 13.10 do not give any information at all about the case of $D J_{K}=$ $\left(\mathbb{C} P^{\infty}, *\right)^{K}$ or $\mathcal{Z}_{K}=\left(D^{2}, S^{1}\right)^{K}$. In the latter case, for example, the join terms $X_{i_{1}} * \cdots * X_{i_{k}}$ are just single spheres. However, Bahri, Bendersky, Cohen and Gitler [2] considered the case of Davis-Januszkiewicz spaces and showed that $D J_{K}$ is elliptic if and only if it is a product of spheres and discs.

Another problem is to consider what happens in the more general case of $(\underline{X}, \underline{A})^{K}$.

Problem 13.13: Determine which polyhedral products $(\underline{X}, \underline{A})^{K}$ are elliptic and which are hyperbolic. 
It may be helpful to approach Problem 13.13 by considering more families of special cases, such as when the $C W$-pairs $\left(X_{i}, A_{i}\right)$ have the property that the homotopy fibre $Y_{i}$ of the inclusion $A_{i} \longrightarrow X_{i}$ has at least two generators in reduced rational homology, or some other special property. Many interesting pairs fall into this case, such as $\left(\mathbb{C} P^{n}, \mathbb{C} P^{k}\right)$ for $1 \leq k<n \leq \infty$.

As for Moore's conjecture, the key idea behind showing the nonexistence of an exponent at any prime $p$ in all the cases we considered was to find a wedge of two spheres retracting off $G_{v} * \Omega X_{v}$ or $G(v) * X_{v}$ for some vertex $v$. The same method should work if the spaces $X_{i}$ are taken to be torsion-free suspensions by using Selick's result [36]. It is natural to ask for a solution more generally.

Problem 13.14: Determine whether $(\underline{X}, \underline{*})^{K},(\underline{C X}, \underline{X})^{K}$, or $(\underline{X}, \underline{A})^{K}$ satisfies Moore's conjecture.

\section{Further problems}

In these notes three trends have emerged regarding the homotopy type of polyhedral products $(\underline{X}, \underline{A})^{K}$ : (i) many of the results apply only for the cases of $(\underline{X}, \underline{*})$ and $(\underline{C X}, \underline{X})$, which happily gives results about the DavisJanuszkiewicz space $D J_{K}$ and the moment-angle complex $\mathcal{Z}_{K}$; (ii) knowing

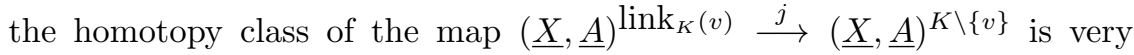
helpful, and (iii) most of the results mentioned are of the form - for certain simplicial complexes $K$ the space $(\underline{C X}, \underline{X})^{K}$ is homotopy equivalent to a wedge of suspensions of iterated smashes of the spaces $X_{i}$.

Let's address these trends in turn. First, identifying the homotopy types of $D J_{K}$ and $\mathcal{Z}_{K}$ are crucial, which is what motivates the generalization to identifying the homotopy types of $(\underline{X}, \underline{*})^{K}$ and $(\underline{C X}, \underline{X})^{K}$. However, there are other interesting polyhedral products and very little has been done to determine their homotopy types.

Problem 14.1: Identify the homotopy type of $(\underline{X}, \underline{A})^{K}$ for pairs of spaces $\left(X_{i}, A_{i}\right)$ where neither $X_{i}$ nor $A_{i}$ is contractible.

Of course, this will probably involve specializing to certain pairs with special properties, and certain simplicial complexes. But at this point almost nothing is known so any progress would be interesting. A curious case to begin with may be pairs $\left(\mathbb{C} P^{n}, \mathbb{C} P^{k}\right)$ for $1 \leq k<n \leq \infty$.

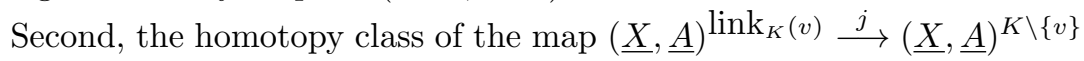
played a pivotal role in the strategy for identifying the homotopy type of 
$(\underline{C X}, \underline{X})^{K}$ for $K$ shifted in Section 7 , and it played an equally important role in identifying hyperbolic polyhedral products in Section 11. Moreover, knowing the homotopy class of $j$ informs on the homotopy type of its fibre $G(v)$, which appears in the homotopy decomposition for $\Omega(\underline{X}, \underline{A})^{K}$.

Problem 14.2: Find other sequences of pairs $(\underline{X}, \underline{A})$ and simplicial complexes $K$ for which the homotopy class of the map $(\underline{X}, \underline{A})^{\operatorname{link}_{K}(v)} \stackrel{j}{\longrightarrow}$ $(\underline{X}, \underline{A})^{K \backslash\{v\}}$ can be identified, and use this to determine information about $(\underline{X}, \underline{A})^{K}$ and $\Omega(\underline{X}, \underline{A})^{K}$.

In particular, a better understanding of the map $j$ would help resolve the problems already stated regarding the elliptic-hyperbolic dichotomy and Moore's conjecture.

Third, most moment-angle complexes $\mathcal{Z}_{K}$ and polyhedral products $(\underline{C X}, \underline{X})^{K}$ are not homotopy equivalent to wedges of suspensions of iterated smashes of the spaces $X_{i}$. In particular, it is known [3] that if $K$ is a polytopal sphere then $\mathcal{Z}_{K}$ is a manifold, and so cannot be homotopy equivalent to a wedge of two or more spaces. In particular, if $K$ is the boundary of an $m$-gon then by $[7,29]$ there is a diffeomorphism

$$
\mathcal{Z}_{K} \simeq \#_{k=3}^{m-1}\left(S^{k} \times S^{m+2-k}\right) \#(k-2)\left(\begin{array}{c}
m-2 \\
k-1
\end{array}\right)
$$

where \# is the connected sum operation. This generalizes to $K$ being the dual of the boundary of certain higher dimensional polytopes. Further generalizations were proved in [15]. This raises several questions.

Problem 14.3: The diffeomorphism with the connected sum in (14.1) was proved using geometric methods. Is there a homotopy-theoretic way of recognizing a space having the homotopy type of a connected sum?

Problem 14.4: If $K$ is as in (14.1) then what is the homotopy type of $(\underline{C X}, \underline{X})^{K}$ in the case of more general pairs $\left(C X_{i}, X_{i}\right)$ ? While not a manifold in general, it would appear to be a useful generalization.

Problem 14.5: What other homotopy types can be realized by a momentangle complex or a polyhedral product?

Some initial progress has been made in Problem 14.3: in [16] it was shown that Whitehead products play an important role in the homotopy class of the map $\mathcal{Z}_{K} \longrightarrow D J_{K}$, and in [39] an intriguing connection was made between the homotopy type of the moment-angle complex $\mathcal{Z}_{K}$ 
in (14.1) and the homotopy type of $m-2$ disjoint points. Nothing is really known about Problem 14.4.

For Problem 14.5, we mention two examples. In [16] it was shown that there is a 6 -vertex triangulation $K$ of $\mathbb{R} P^{2}$ with the property that $\mathcal{Z}_{K}$ has the Moore space $P^{9}(2)$ as a wedge summand. In particular, there is a nontrivial $S q^{1}$ operation in the mod-2 cohomology of $\mathcal{Z}_{K}$. In [27] it was shown that there is a 9 -vertex triangulation $L$ of $\mathbb{C} P^{2}$ with the property that $\mathcal{Z}_{L}$ has $\Sigma^{10} \mathbb{C} P^{2}$ as a wedge summand. In particular, there is a nontrivial $S q^{2}$ operation in the mod-2 cohomology of $\mathcal{Z}_{L}$. Connected to Problem 14.5 then, is the following problem.

Problem 14.6: For which simplicial complexes $K$ does $\mathcal{Z}_{K}$ have nontrivial Steenrod operations in mod-p cohomology? In particular, for which simplicial complexes $K$ does $\mathcal{Z}_{K}$ have mod-p torsion in cohomology?

\section{Acknowledgments}

These notes were prepared for a lecture series as part of the programme, "Combinatorial and Toric Homotopy", held at the IMS in Singapore in August 2015. I would like to thank the IMS for its support; the organizers, Jelena Grbić, Zhi Lü and Jie Wu, for inviting me to give these talks; and Alastair Darby for carefully proofreading the notes.

\section{References}

1. A. Bahri, M. Bendersky, F.R. Cohen and S. Gitler, The polyhedral product functor: a method of decomposition for moment-angle complexes, arrangements and related spaces, Adv. Math. 225 (2010), 1634-1668.

2. A. Bahri, M. Bendersky, F.R. Cohen and S. Gitler, On the rational homotopy type of moment-angle complexes, Proc. Steklov Inst. Math. 286 (2014), 219223.

3. P. Beben and J. Grbić, Configuration spaces and polyhedral products, arXiv:1409.4462.

4. M.G. Barratt, Spaces of finite characteristic, Quart. J. Math. Oxford 11 (1960), 124-136.

5. V.M. Buchstaber and T.E. Panov, Torus actions and their applications in topology and combinatorics, University Lecture Series 24, American Mathematical Society, 2002.

6. V.M. Buchstaber and T.E. Panov, Toric topology, Mathematical Surveys and Monographs 204, American Mathematical Society, 2015.

7. F. Bosio and L. Meersseman, Real quadrics in $\mathbb{C}^{n}$, complex manifolds and convex polytopes, Acta Math. 197 (2006), 53-127. 
8. W. Chachólski, W. Pitsch, J. Scherer and D. Stanley, Homotopy exponents for large $H$-spaces, Int. Math. Res. Not. IMRN 2008, 16, Art. ID rnn061, $5 \mathrm{pp}$.

9. F.R. Cohen, A course in some aspects of classical homotopy theory, Lecture Notes in Math. 1286, Springer-Verlag (1987), 1-92.

10. M.W. Davis and T. Januszkiewicz, Convex polytopes, Coxeter orbifolds and torus actions, Duke Mathematical Journal 62 (1991), 417-452.

11. G. Denham and A.I. Suciu, Moment-angle complexex, monomial ideals, and Massey products, Pure Appl. Math. Q. 3 (2007), 25-60.

12. Y. Félix, S. Halperin and J.-C. Thomas, Elliptic spaces II. Enseign. Math. (2) 39 (1993), 25-32.

13. Y. Félix and D. Tanré, Rational homotopy of the polyhedral product functor, Proc. Amer. Math. Soc. 137 (2009), 891-898.

14. T. Ganea, A generalization of the homology and homotopy suspension, Comment. Math. Helv. 39 (1965), 295-322.

15. S. Gitler and S. Lopez de Medrano, Intersections of quadrics, moment-angle manifolds and connected sums, Geom. Topol. 17 (2013), 1497-1534.

16. J. Grbić, T. Panov, S. Theriault and J. Wu, The homotopy types of momentangle complexes for flag complexes, Trans. Amer. Math. Soc. 368 (2016), 6663-6682.

17. J. Grbić and S. Theriault, The homotopy type of the complement of the codimension two coordinate subspace arrangement, Russian Math. Surveys 59 (2005), 203-204.

18. J. Grbić and S. Theriault, The homotopy type of the complement of a coordinate subspace arrangement, Topology 46 (2007), 357-396.

19. J. Grbić and S. Theriault, The homotopy type of the polyhedral product for shifted complexes, Adv. Math. 245 (2013), 690-715.

20. J. Grbić and S. Theriault, Homotopy theory in toric topology, Russian Math. Surveys 71 (2016), 185-251.

21. V. Grujić and V. Welker, Moment-angle complexes of pairs $\left(D^{n}, S^{n-1}\right)$ and simplicial complexes with vertex-decomposable duals, Monatsh. Math. 176 (2015), 255-273.

22. K. Iriye and D. Kishimoto, Decompositions of polyhedral products, Adv. Math. 245 (2013), 716-736.

23. K. Iriye and D. Kishimoto, Fat wedge filtrations and decomposition of polyhedral products, arXiv:1412.4866.

24. K. Iriye and D. Kishimoto, Polyhedral products for shifted complexes and higher Whitehead products, arXiv:1505.04892.

25. I.M. James, Reduced product spaces, Ann. of Math. 62 (1955), 170-197.

26. I.M. James, The suspension triad of a sphere, Ann. of Math. 63 (1956), 407-429.

27. I. Limonchenko, Combinatorial commutative algebra and topology of momentangle complexes, $\mathrm{PhD}$ thesis, Moscow State University, 2015.

28. J. Long, Thesis, Princeton University, 1978.

29. D. MacGavran, Adjacent connected sums and torus actions, Trans. Amer. Math. Soc. 251 (1979), 235-254. 
30. M. Mather, Pull-backs in homotopy theory, Canad. J. Math. 28 (1976), 225263.

31. C.A. McGibbon and C.W. Wilkerson, Loop spaces of finite complexes at large primes, Proc. Amer. Math. Soc. 96 (1986), 698-702.

32. J.A. Neisendorfer, Primary Homotopy Theory, Mem. Amer. Math. Soc. 232 (1980).

33. J.A. Neisendorfer, The exponent of a Moore space. Algebraic topology and algebraic K-theory (Princeton, N.J., 1983), 35-71, Ann. of Math. Stud. 113, Princeton Univ. Press, Princeton, NJ, 1987.

34. J. Neisendorfer and P. Selick, Some examples of spaces with and without homotopy exponents, Current trends in algebraic topology, Part 1 (London, Ont., 1981), pp. 343-357, CMS Conf. Proc. 2, Amer. Math. Soc., Providence, R.I., 1982.

35. G.J. Porter, The homotopy groups of wedges of suspensions, Amer. J. Math. 88 (1966), 655-663.

36. P. Selick, On conjectures of Moore and Serre in the case of torsion-free suspensions, Math. Proc. Cambridge Philos. Soc. 94 (1983), 53-60.

37. M. Stelzer, Hyperbolic spaces at large primes and a conjecture of Moore, Topology 43 (2004), 667-675.

38. S. Theriault, Homotopy exponents of $\bmod 2^{r}$ Moore spaces, Topology 47 (2008), 369-398.

39. S. Theriault, Moment-angle manifolds and Panov's problem, Int. Math. Res. Not. (2015), doi:10.1093/imrn/rnu281.

40. S. Theriault, The dual polyhedral product, cocategory and nilpotence, arXiv:1506.05998.

41. H. Toda, On the double suspension $E^{2}$, J. Inst. Polytech. Osaka City Univ., Ser. A 7 (1956), 103-145. 